\title{
Harris Hawks Optimization: Algorithm and Applications
}

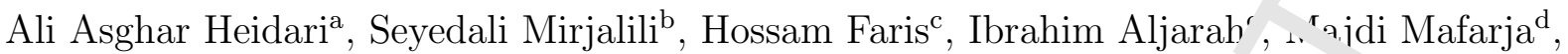 \\ Huiling Chen *e \\ ${ }^{a}$ School of Surveying and Geospatial Engineering, University of Tehra ${ }_{\llcorner}, T$, aran, Iran \\ as_heidari@ut.ac.ir \\ ${ }^{b}$ School of Information and Communication Technology, Griffith University, Nrthai, Brisbane, QLD 4111, \\ Australia \\ seyedali.mirjalili@griffithuni.edu.au \\ ${ }^{c}$ King Abdullah II School for Information Technology, The Universit» of « dan, Amman, Jordan \\ $\{$ i.aljarah,hossam.faris\}@ju.edu.jo \\ ${ }^{d}$ Department of Computer Science, Birzeit University, POBo. 14, W, st Bank, Palestine \\ mmafarja@birzeit.edu \\ ${ }^{e}$ Department of Computer Science, Wenzhou Univers ty, I' ${ }^{2}$ thou 325035, China \\ chenhuiling.jlu@gmail.com
}

\begin{abstract}
In this paper, a novel population-based, nature-inspireu ntimization paradigm is proposed, which is called Harris Hawks Optimizer (HHO). The ma $\eta 11$ ation of HHO is the cooperative behavior and chasing style of Harris' hawks in nature allec surprise pounce. In this intelligent strategy, several hawks cooperatively pounce a prey fro ' ' ':fferent directions in an attempt to surprise it. Harris hawks can reveal a variety of chasi n nat arns based on the dynamic nature of scenarios and escaping patterns of the prey. This work athematically mimics such dynamic patterns and behaviors to develop an optimization alonrithm. The effectiveness of the proposed HHO optimizer is checked, through a comparison with sther ı. ature-inspired techniques, on 29 benchmark problems and several real-world engineering pro 'oms The statistical results and comparisons show that the HHO algorithm provides very pr mising and occasionally competitive results compared to well-established metaheuristic tec ${ }_{2}>{ }_{1}$,ues
\end{abstract}

Keywords:

Nature-inspired computing, Harris in wks optimization algorithm, Swarm intelligence, Optimization, Metaheuristi

\section{Introduction}

Many real-world probl $>$ ms in machine learning and artificial intelligence have generally a continuous, discrete, a nstrai ed or unconstrained nature $[1,2]$. Due to these characteristics, it is hard to tackle so ${ }^{*}$. claswes of problems using conventional mathematical programming approaches such as conjug; te gra ient, sequential quadratic programming, fast steepest, and quasi-Newton methods [3, 4]. S ver. types of research have verified that these methods are not efficient enough or always ef, 'in.' in dealing with many larger-scale real-world multimodal, non-continuous, and non-differentia e problems [5]. Accordingly, metaheuristic algorithms have been designed and utilized for tacklıng many problems as competitive alternative solvers, which is because of their

\footnotetext{
*Corresponding author: Huiling Chen (chenhuiling.jlu@gmail.com)
} 
simplicity and easy implementation process. In addition, the core operations of these methods do not rely on gradient information of the objective landscape or its mathemati al traits. However, the common shortcoming for the majority of metaheuristic algorithms is they often show a delicate sensitivity to the tuning of user-defined parameters. Another drawbuzk is that the metaheuristic algorithms may not always converge to the global optimur .. [ ]

In general, metaheuristic algorithms have two types [7]; single solutı, sased (i.g. Simulated Annealing (SA) [8]) and population-based (i.g. Genetic Algorithm (GA, [9]). As the name indicates, in the former type, only one solution is processed during the op imization phase, while in the latter type, a set of solutions (i.e. population) are evolved in ack iteration of the optimization process. Population-based techniques can often find an optimal os uboptimal solution that may be same with the exact optimum or located in its neighl orhoos. Population-based metaheuristic (P-metaheuristics) techniques mostly mimic natural pı nor ena $[10,11,12,13]$. These algorithms start the optimization process by generating a et ( $r$-nulation) of individuals, where each individual in the population represents a candidate solu : $\mathrm{il}$ to he optimization problem. The population will be evolved iteratively by replacing the curı pupulation with a newly generated population using some often stochastic operators $[14,15]$. The optimization process is proceeded until satisfying a stopping criteria (i.e. maximum nui her $f$ : erations) $[16,17]$.

Based on the inspiration, P-metaheuristics can be cate rized in four main groups $[18,19]$ (see Fig. 1): Evolutionary Algorithms (EAs), Physics-b sed, Human-based, and Swarm Intelligence (SI) algorithms. EAs mimic the biological evoluti marv beilaviors such as recombination, mutation, and selection. The most popular EA is the GA tha $r$ imics the Darwinian theory of evolution [20]. Other popular examples of EAs are Differenti. F, Fo tion (DE) [21], Genetic Programming (GP) [20], and Biogeography-Based Optimizer (BBO) 24 . Physics-based algorithms are inspired by the physical laws. Some examples of these algoı ' Force Optimization (CFO) [24], and Gravitational Search Algorithm (GSA) [25]. Salcedo-Sanz [26] has deeply reviewed several physir wan optimizers. The third category of P-metaheuristics includes the set of algorithms that m. mic so ne human behaviors. Some examples of the humanbased algorithms are Tabu Search ( $\mathrm{IS}$ ) $L^{-1}$, Socio Evolution and Learning Optimization (SELO) [28], and Teaching Learning Based $\mathrm{Jp}^{+}$mi ation(TLBO) [29]. As the last class of P-metaheuristics, SI algorithms mimic the social $r$ ehav $>$ r (e.g. decentralized, self-organized systems) of organisms living in swarms, flocks, or he $\mathrm{u}\lceil 30,31]$. For instance, the birds flocking behaviors is the main inspiration of the Particle Swarm ( In PSO, each particle in th $\&$ sv arm represents a candidate solution to the optimization problem. In the optimization process, ch particle is updated with regard to the position of the global best particle and its own (lo al) best position. Ant Colony Optimization (ACO) [33], Cuckoo Search (CS) [34], and Artificia. B e C slony (ABC) are other examples of the SI techniques.

Regardless of the variety of these algorithms, there is a common feature: the searching steps have two phases: $\epsilon$ cplorat on (diversification) and exploitation (intensification) [26]. In the exploration phase, the ${ }^{1} \mathrm{~m}$. thm should utilize and promote its randomized operators as much as possible to dee 1 y exp 'ore various regions and sides of the feature space. Hence, the exploratory behaviors of a ell-de, igned optimizer should have an enriched-enough random nature to efficiently alloc ${ }^{+n}$ more randomly-generated solutions to different areas of the problem topography during early s, ir s of the searching process [35]. The exploitation stage is normally performed after the exploration, hase. In this phase, the optimizer tries to focus on the neighborhood of betterquality solutions located inside the feature space. It actually intensifies the searching process in a local region instead of all-inclusive regions of the landscape. A well-organized optimizer should be capable of making a reasonable, fine balance between the exploration and exploitation tenden- 


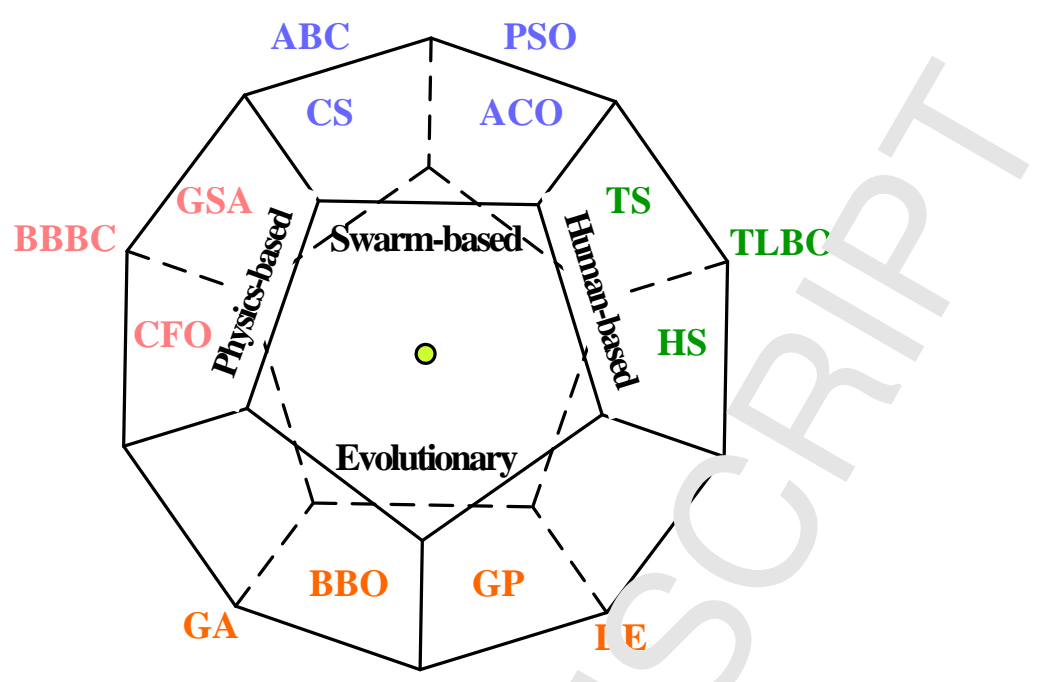

Figure 1: Classification of meta-heuristic techniques ( $m_{1}$ ta-heuristic diamond)

cies. Otherwise, the possibility of being trapped in lnnal n. ima (LO) and immature convergence drawbacks increases.

We have witnessed a growing interest and $\varepsilon$ vm noss in the successful, inexpensive, efficient application of EAs and SI algorithms in recent yea. . However, referring to No Free Lunch (NFL) theorem [36], all optimization algorithms pre sea so-far show an equivalent performance on average if we apply them to all possible optimizar on tasks. According to NFL theorem, we cannot theoretically consider an algorithm as a gene 9.1 -purpose universally-best optimizer. Hence, NFL theorem encourages searching for developing more efficient optimizers. As a result of NFL theorem, besides the widespread studies on the ticar', performance aspects and results of traditional EAs and SI algorithms, new optimizers wi: speci ic global and local searching strategies are emerging in recent years to provide more var ety of c ioices for researchers and experts in different fields.

In this paper, a new nature-in nir $d$ or cimization technique is proposed to compete with other optimizers. The main idea bet nd $t_{1}$ proposed optimizer is inspired from the cooperative behaviors of one of the most int $\mathrm{H}_{0}$ 'nt birds, Harris' Hawks, in hunting escaping preys (rabbits in most cases) [37]. For this pumse, a new mathematical model is developed in this paper. Then, a stochastic metaheuristic is desi ,ned based on the proposed mathematical model to tackle various optimization problems.

The rest of this reser $\mathrm{ch}$ is organized as follows. Section 2 represents the background inspiration and info about the coope tivr life of Harris' hawks. Section 3 represents the mathematical model and computational rocerures of the HHO algorithm. The results of HHO in solving different benchmark and rea. world ase studies are presented in Section 4 Finally, Section 6 concludes the work with some rofu ${ }_{1}$, spectives.

\section{Backgrounc}

In 1997, Lc ' ' ', Lefebvre proposed an approach to measure the avian "IQ" based on the observed innovations in $\mathrm{ft}$ ling behaviors [38]. Based on his studies [38, 39, 40, 41], the hawks can be listed amongst the most intelligent birds in nature. The Harris' hawk (Parabuteo unicinctus) is a wellknown bird of prey that survives in somewhat steady groups found in southern half of Arizona, USA [37]. Harmonized foraging involving several animals for catching and then, sharing the slain 
animal has been persuasively observed for only particular mammalian carnivores. The Harris's hawk is distinguished because of its unique cooperative foraging activities ${ }^{+}$gether with other family members living in the same stable group while other raptors usual, attack to discover and catch a quarry, alone. This avian desert predator shows evolved innovativ team chasing capabilities in tracing, encircling, flushing out, and eventually attacki $g$ t ie potential quarry. These smart birds can organize dinner parties consisting of several indivia 's in the non-breeding season. They are known as truly cooperative predators in the raptor "ealni. As reported by Bednarz [37] in 1998, they begin the team mission at morning tr ${ }_{11 l_{\xi}} \mathrm{ht}$, with leaving the rest roosts and often perching on giant trees or power poles inside their hor ıe realm. They know their family members and try to be aware of their moves during the att $\sim \mathrm{k}$. 'Then assembled and party gets started, some hawks one after the other make short tou s and then, land on rather high perches. In this manner, the hawks occasionally will perform . "les pfrog" motion all over the target site and they rejoin and split several times to activel sea $^{-h}$ for the covered animal, which is usually a rabbit ${ }^{2}$.

The main tactic of Harris' hawks to capture a prey is _.rpise pounce", which is also known as "seven kills" strategy. In this intelligent strategy, everal lawks try to cooperatively attack from different directions and simultaneously converg on a dected escaping rabbit outside the cover. The attack may rapidly be completed by capturin the surprised prey in few seconds, but occasionally, regarding the escaping capabilities an' hehaviors of the prey, the seven kills may include multiple, short-length, quick dives neark - the prey during several minutes. Harris' hawks can demonstrate a variety of chasing styles depe. $\mathrm{d}$ nt on the dynamic nature of circumstances and escaping patterns of a prey. A switchins nctic occurs when the best hawk (leader) stoops at the prey and get lost, and the chase will be ontinued by one of the party members. These switching activities can be observed in differ 'nt, su wations because they are beneficial for confusing the escaping rabbit. The main advantage of thuse cooperative tactics is that the Harris' hawks can pursue the detected rabbit to ex' au $^{\prime}$ 'on, which increases its vulnerability. Moreover, by perplexing the escaping prey, it car 'nt rec ver its defensive capabilities and finally, it cannot escape from the confronted team br siege :" se one of the hawks, which is often the most powerful and experienced one, effortlessly c apt $\mathrm{r}$ res ' he tired rabbit and shares it with other party members. Harris' hawks and their main br 'navı" "s an be seen in nature, as captured in Fig. 2.

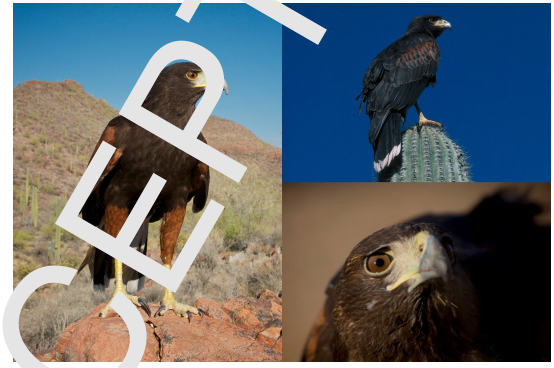

(a) Parabuteo unicinctus

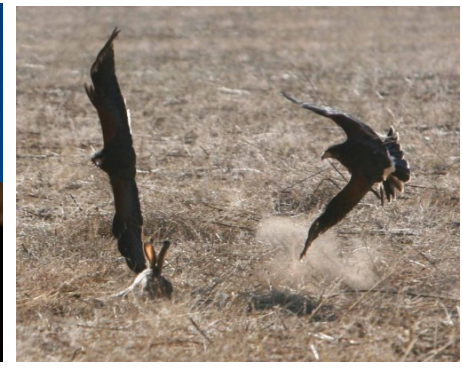

(b) Surprise pounce

Figure 2: Harris's hawk and their behaviors ${ }^{3}$

\footnotetext{
${ }^{2}$ Interested readers can refer to the following documentary videos: (a) https://bit.ly/2Qew2qN, (b) https: //bit.1y/2qsh8Cl, (c) https://bit.1y/2P7OMvH, (d) https://bit.ly/2DosJdS

${ }^{3}$ These images were obtained from (a) https://bit.1y/2qAsODb (b) https://bit.ly/2zBFo91
} 


\section{Harris hawks optimization (HHO)}

In this section, we model the exploratory and exploitative phases of th proposed HHO inspired by the exploring a prey, surprise pounce, and different attacking straiegies of Harris hawks. HHO is a population-based, gradient-free optimization technique; hence, $\sim$, n be applied to any optimization problem subject to a proper formulation. Figure 3 shows . 1 r iases of HHO, which are described in the next subsections.

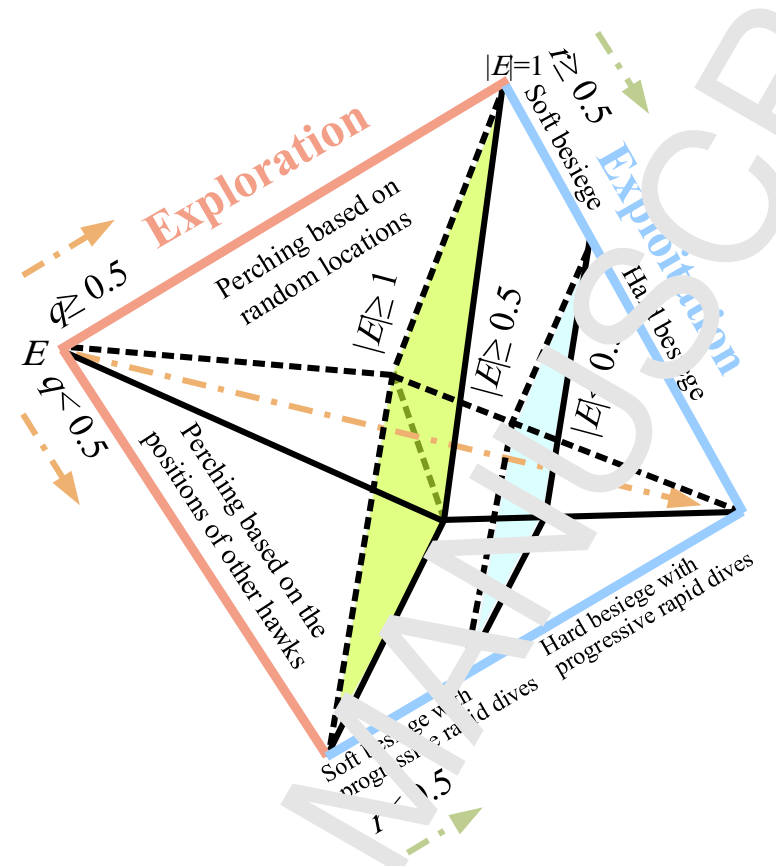

Figur s: - ifferent phases of HHO

\subsection{Exploration phase}

In this part, the exploratio mec $\mathrm{c}_{-}$ism of HHO is proposed. If we consider the nature of Harris' hawks, they can track aı d detect the prey by their powerful eyes, but occasionally the prey cannot be seen easily. $\mathrm{H} \wedge$ nce, the hawks wait, observe, and monitor the desert site to detect a prey maybe after several sou s. In HHO, the Harris' hawks are the candidate solutions and the best candidate solution in eac step is considered as the intended prey or nearly the optimum. In HHO, the Harris' hawk per 'h randomly on some locations and wait to detect a prey based on two strategies. If we conside. in $\mathrm{f}$ qual chance $q$ for each perching strategy, they perch based on the positions of other fo . nllly meınbers (to be close enough to them when attacking) and the rabbit, which is modeled in Eq. ( ) for the condition of $q<0.5$, or perch on random tall trees (random locations inside thr $g_{\perp}, \mathrm{s}$ home range), which is modeled in Eq. (1) for condition of $q \geq 0.5$.

$$
X\left(\bullet\llcorner 1)=\left\{\begin{array}{cl}
X_{\text {rand }}(t)-r_{1}\left|X_{\text {rand }}(t)-2 r_{2} X(t)\right| & q \geq 0.5 \\
\left(X_{\text {rabbit }}(t)-X_{m}(t)\right)-r_{3}\left(L B+r_{4}(U B-L B)\right) & q<0.5
\end{array}\right.\right.
$$

where $X\left(t+1\right.$, is the position vector of hawks in the next iteration $t, X_{\text {rabbit }}(t)$ is the position of rabbit, $X(t)$ is the current position vector of hawks, $r_{1}, r_{2}, r_{3}, r_{4}$, and $q$ are random numbers inside $(0,1)$, which are updated in each iteration, $L B$ and $U B$ show the upper and lower bounds of variables, $X_{\text {rand }}(t)$ is a randomly selected hawk from the current population, and $X_{m}$ is the average 
position of the current population of hawks. We proposed a simple model to generate random locations inside the group's home range $(L B, U B)$. The first rule generates s slutions based on a random location and other hawks. In second rule of Eq. (1), we have the diff i nce of the location of best so far and the average position of the group plus a randomly-scaler comp nent based on range of variables, while $r_{3}$ is a scaling coefficient to further increase th: ra idom nature of rule once $r_{4}$ takes close values to 1 and similar distribution patterns may occu. in this rule, we add a randomly scaled movement length to the $L B$. Then, we considered a ranc $m$ scaling coefficient for the component to provide more diversification trends and explore $d$. ste ent regions of the feature space. It is possible to construct different updating rules, but we $\mathrm{i}^{\mathrm{i}} \mathrm{li}_{\mathrm{i}} \mathrm{d}$ the simplest rule, which is able to mimic the behaviors of hawks. The average position of h $2 w k_{s}:_{s}$ attained using Eq. (2):

$$
X_{m}(t)=\frac{1}{N} \sum_{i=1}^{N} X_{i}(t)
$$

where $X_{i}(t)$ indicates the location of each hawk in iteration ' and $N$ denotes the total number of hawks. It is possible to obtain the average location in 'ifferent ways, but we utilized the simplest rule.

\subsection{Transition from exploration to exploital' $n$}

The HHO algorithm can transfer from explc w.'n to exploitation and then, change between different exploitative behaviors based on the esca ${ }_{1}$. . $g$ energy of the prey. The energy of a prey decreases considerably during the escaping be " $a$ ior. To model this fact, the energy of a prey is modeled as:

$$
E=: F_{n}\left(1-\frac{t}{T}\right)
$$

where $E$ indicates the escaping energy or ' $E_{0}$ is the initial state of its energy. $-H H O E_{0}$ randomly changes inside the interval $(-1,1)$ at each iteration. When the value of $E_{0}$ a " sases from 0 to -1, the rabbit is physically flagging, whilst when the value of $E_{0}$ inc sasr $s$ fr $>m$ to 1 , it means that the rabbit is strengthening. The dynamic escaping energy $E$ has $\mathrm{d}$ creasing trend during the iterations. When the escaping energy $|E| \geq 1$, the hawks sea ¿ different regions to explore a rabbit location, hence, the HHO performs the exploration phase, ana when $|E|<1$, the algorithm try to exploit the neighborhood of the solutions during the exp jitation steps. In short, exploration happens when $|E| \geq 1$, while exploitation happens in law "steps when $|E|<1$. The time-dependent behavior of $E$ is also demonstrated in Fig. 4

\subsection{Exploitation - hase}

In this phase, t te Har is' hawks perform the surprise pounce (seven kills as called in [37])

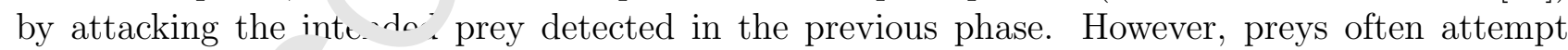
to escape from dang ous situations. Hence, different chasing styles occur in real situations. According to th escar ng behaviors of the prey and chasing strategies of the Harris' hawks, four possible stra ${ }^{2}$ mies are proposed in the HHO to model the attacking stage.

The preys vays try to escape from threatening situations. Suppose that $r$ is the chance of a prey in successfu "ly escaping $(r<0.5)$ or not successfully escaping $(r \geq 0.5)$ before surprise pounce. Whatever the prey does, the hawks will perform a hard or soft besiege to catch the prey. It means that they will encircle the prey from different directions softly or hard depending on the retained energy of the prey. In real situations, the hawks get closer and closer to the intended prey to 


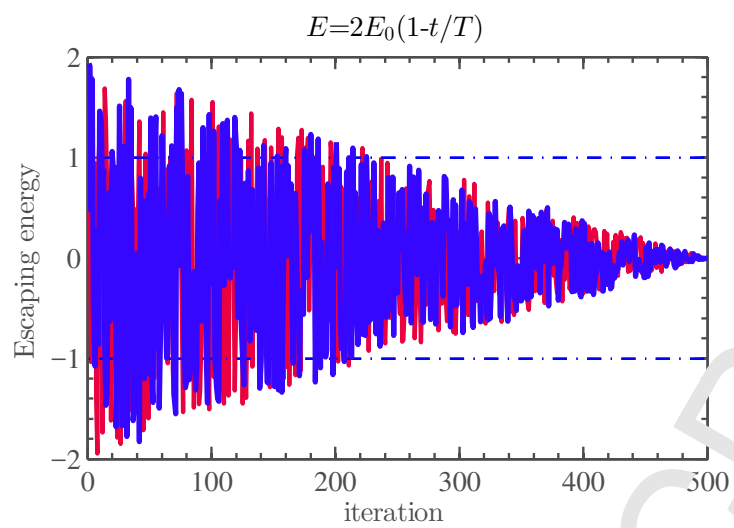

Figure 4: Behavior of $E$ during two runs and 50 iter „ons

increase their chances in cooperatively killing the rabbit $k_{*}$ perfor .ing the surprise pounce. After several minutes, the escaping prey will lose more and more es 'rgy; then, the hawks intensify the besiege process to effortlessly catch the exhausted prey. To $r$ odel this strategy and enable the HHO to switch between soft and hard besiege processes, the $E$ parameter is utilized.

In this regard, when $|E| \geq 0.5$, the soft besiege ' xprusio, and when $|E|<0.5$, the hard besiege occurs.

\subsubsection{Soft besiege}

When $r \geq 0.5$ and $|E| \geq 0.5$, the rabbit s' 1 has enough energy, and try to escape by some random misleading jumps but finally it carnnt L ' ring these attempts, the Harris' hawks encircle it softly to make the rabbit more exhausted $a_{\iota .} d$ then perform the surprise pounce. This behavior is modeled by the following rules:

$$
\begin{gathered}
X(t+1)=\wedge X(+)-E\left|J X_{\text {rabbit }}(t)-X(t)\right| \\
\Delta \cdot(t)=X_{\text {rabbit }}(t)-X(t)
\end{gathered}
$$

where $\Delta X(t)$ is the difference $r$ etwee ne position vector of the rabbit and the current location in iteration $t, r_{5}$ is a random un anside $(0,1)$, and $J=2\left(1-r_{5}\right)$ represents the random jump strength of the rabbit throu '.- ' u the escaping procedure. The $J$ value changes randomly in each iteration to simulate the $\mathrm{n}$.tur of rabbit motions.

\subsubsection{Hard besiege}

When $r \geq 0.5$ and $\mid \perp^{\prime}<0$, the prey is so exhausted and it has a low escaping energy. In addition, the Harris' alawk hardly encircle the intended prey to finally perform the surprise pounce. In this situation, th curre it positions are updated using Eq. (6):

$$
X(t+1)=X_{\text {rabbit }}(t)-E|\Delta X(t)|
$$

A simple oxampic of this step with one hawk is depicted in Fig. 5.

\subsubsection{Soft $b$, siege with progressive rapid dives}

When still $|E| \geq 0.5$ but $r<0.5$, the rabbit has enough energy to successfully escape and still a soft besiege is constructed before the surprise pounce. This procedure is more intelligent than the previous case. 


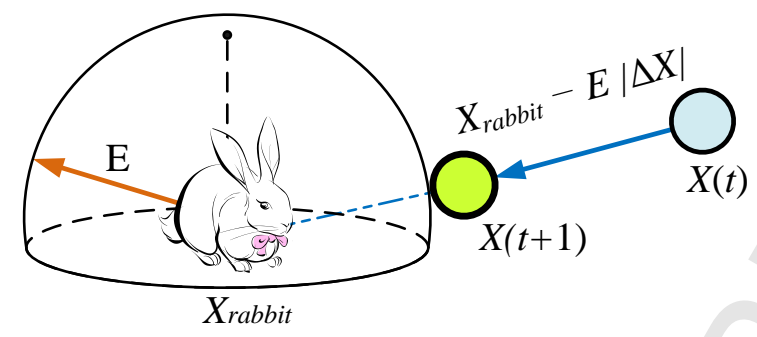

Figure 5: Example of overall vectors in the case of hà ${ }^{1} 1$ esiege

To mathematically model the escaping patterns of the prey ar. leapt og movements (as called in [37]), the levy flight (LF) concept is utilized in the HHO algor vitm. I ne LF is utilized to mimic the real zigzag deceptive motions of preys (particularity rabbits $d$.rin , escaping phase and irregular, abrupt, and rapid dives of hawks around the escaping pre, Act . ly, hawks perform several team rapid dives around the rabbit and try to progressively correc their location and directions with regard to the deceptive motions of prey. This mechanis. is: so supported by real observations in other competitive situations in nature. It has been cu firmed that LF-based activities are the optimal searching tactics for foragers/predators $i_{i}$ ' Ion-aestructive foraging conditions [42, 43]. In addition, it has been detected the LF-based pattern an be detected in the chasing activities of animals like monkeys and sharks [44, 45, 46, 7]. 1 within this phase of $\mathrm{HHO}$ technique.

Inspired by real behaviors of hawks, we sur ou n that they can progressively select the best possible dive toward the prey when they in $t_{1}$ catch the prey in the competitive situations. Therefore, to perform a soft besiege, we suppos $d$ that the hawks can evaluate (decide) their next move based on the following rule in Ea (7):

$$
Y=X_{\text {rabbu }}(t)-E\left|J X_{\text {rabbit }}(t)-X(t)\right|
$$

Then, they compare the possible $\mathrm{i}$ s' $i$ of such a movement to the previous dive to detect that will it be a good dive or not. If it $\mathrm{w}$ s not + ssonable (when they see that the prey is performing more deceptive motions), they also sar ${ }^{+}$o perform irregular, abrupt, and rapid dives when approaching the rabbit. We supposed th they will dive based on the LF-based patterns using the following rule:

$$
Z=Y+S \times L F(D)
$$

where $D$ is the dimens ,n , $\mathrm{p}$ sblem and $S$ is a random vector by size $1 \times D$ and LF is the levy flight function, which is caı " ated using Eq. (9) [48]:

$$
L_{, H}(x)=0.01 \times \frac{u \times \sigma}{|v|^{\frac{1}{\beta}}}, \sigma=\left(\frac{\Gamma(1+\beta) \times \sin \left(\frac{\pi \beta}{2}\right)}{\left.\Gamma\left(\frac{1+\beta}{2}\right) \times \beta \times 2^{\left(\frac{\beta-1}{2}\right)}\right)}\right)^{\frac{1}{\beta}}
$$

where $u, v \varepsilon$ - $\cdots n$ ndom values inside $(0,1), \beta$ is a default constant set to 1.5.

Hence, the fi.lal strategy for updating the positions of hawks in the soft besiege phase can be performed by $\mathrm{E}_{4}$ (10):

$$
X(t+1)= \begin{cases}Y & \text { if } F(Y)<F(X(t)) \\ Z & \text { if } F(Z)<F(X(t))\end{cases}
$$


where $Y$ and $Z$ are obtained using Eqs.(7) and (8).

A simple illustration of this step for one hawk is demonstrated in Fig. 6. Note that the position history of LF-based leapfrog movement patterns during some iterat: ${ }_{\checkmark}<$ are also recorded and shown in this illustration. The colored dots are the location footprints of LF- Jased patterns in one trial and then, the HHO reaches to the location $Z$. In each step. onl the better position $Y$ or $Z$ will be selected as the next location. This strategy is applied to a." ,earch agents.

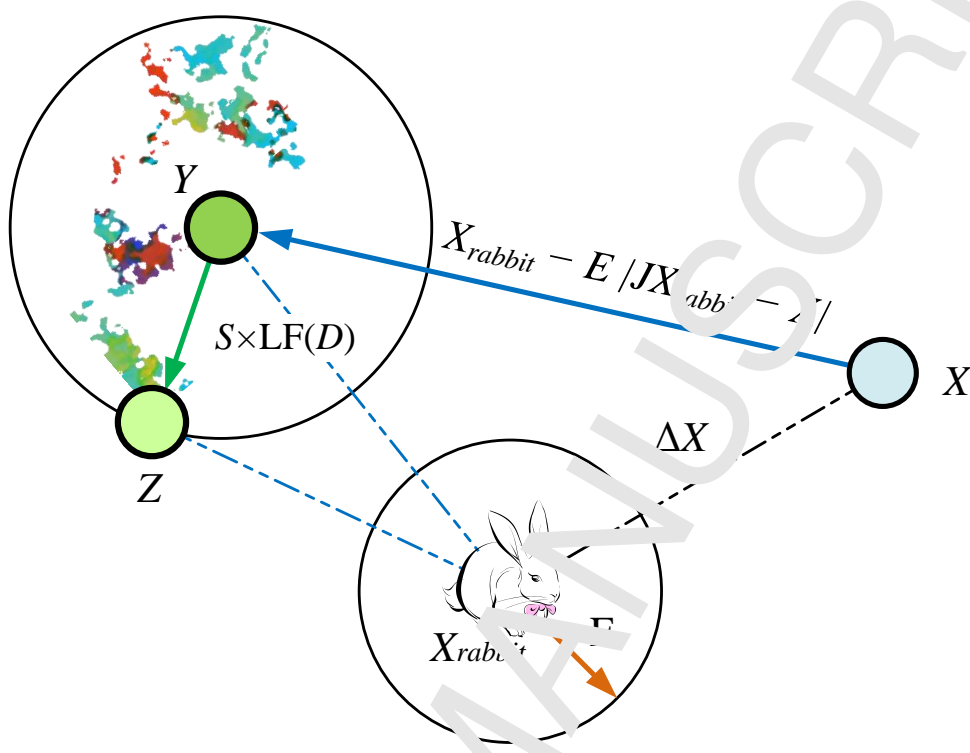

Figure 6: Example of overall vectors in 'e caun of soft besiege with progressive rapid dives

\subsubsection{Hard besiege with progre sive $\mathrm{r}$ เpid dives}

When $|E|<0.5$ and $r<0.5$, the iabbı, ${ }^{h}$ ss not enough energy to escape and a hard besiege is constructed before the surprise pc $\mathrm{Inc}^{\prime}$ to atch and kill the prey. The situation of this step in the prey side is similar to that in the sol nes age, but this time, the hawks try to decrease the distance of their average location with $t^{*}$. escaping prey. Therefore, the following rule is performed in hard besiege condition:

$$
\therefore(t+1)= \begin{cases}Y & \text { if } F(Y)<F(X(t)) \\ Z & \text { if } F(Z)<F(X(t))\end{cases}
$$

where $Y$ and $Z$ are sbtr ned using new rules in Eqs.(12) and (13).

$$
\begin{gathered}
Y=X_{\text {rabbit }}(t)-E\left|J X_{\text {rabbit }}(t)-X_{m}(t)\right| \\
Z=Y+S \times L F(D)
\end{gathered}
$$

where $X_{m}(t)$ is Jbtain d using Eq. (2). A simple example of this step is demonstrated in Fig. 7. Note that the cc'ned lots are the location footprints of LF-based patterns in one trial and only

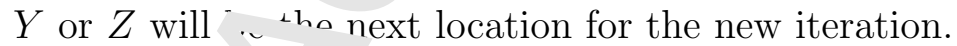

\subsection{Pseudoce te of $\mathrm{HHO}$}

The pseudocode of the proposed HHO algorithm is reported in Algorithm 1. 


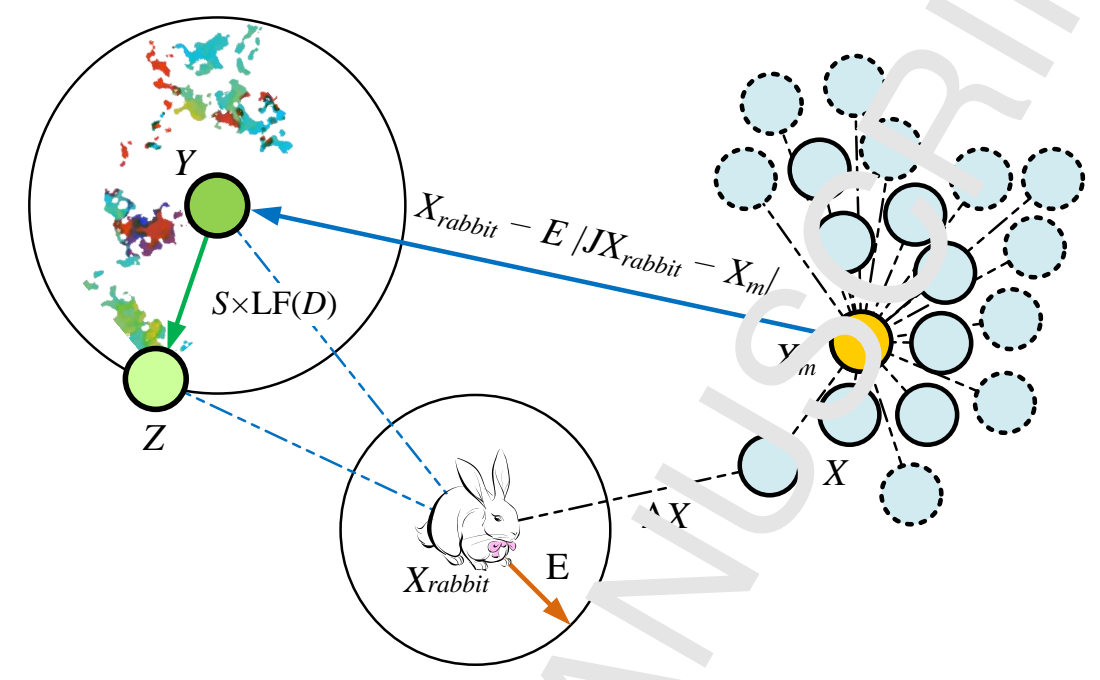

(a) The prncess . 2D space

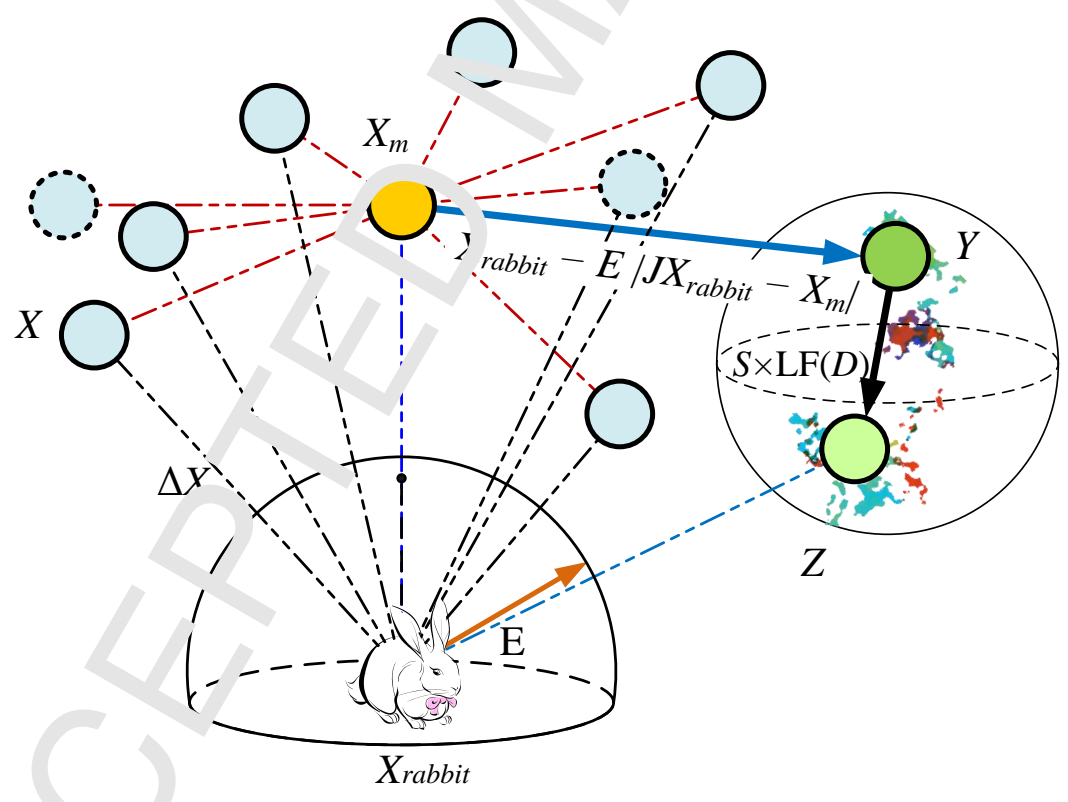

(b) The process in 3D space

Figure 7: Example of ove' 1 ll vectors in the case of hard besiege with progressive rapid dives in 2D and 3D space. 


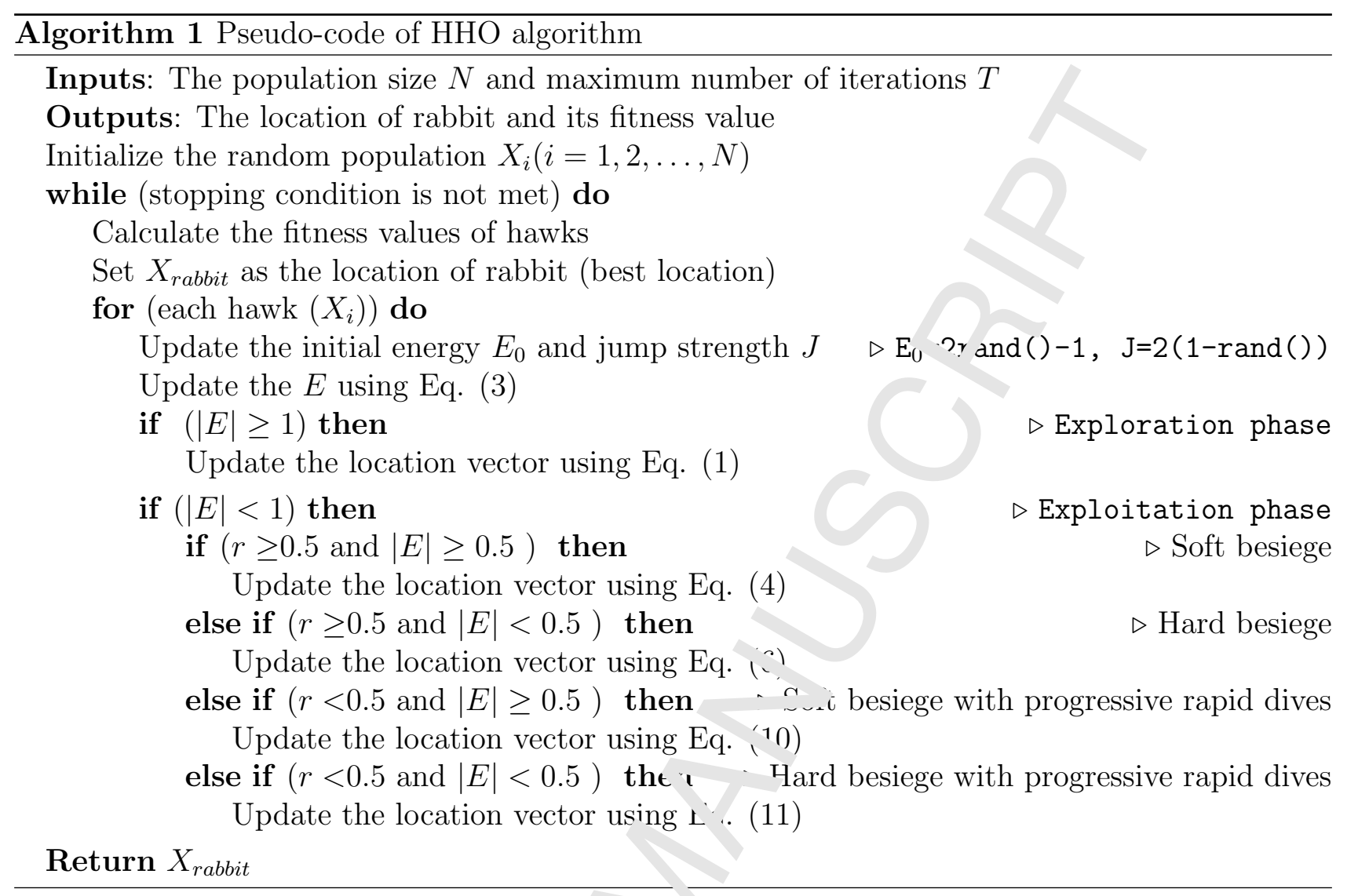

\subsection{Computational complexity}

Note that the computational comp' sxity $\iota^{\complement}$ the HHO mainly depends on three processes: initialization, fitness evaluation, and updatı. of 'awks. Note that with $N$ hawks, the computational complexity of the initialization pr $\mathrm{uces}^{3}$ is $\mathcal{O}(N)$. The computational complexity of the updating mechanism is $\mathrm{O}(T \times N)+\mathrm{O}(T, N \times D$, which is composed of searching for the best location and updating the location vec or of a. hawks, where $T$ is the maximum number of iterations and $D$ is the dimension of $\mathrm{s}_{1}^{\prime}$ ech problems. Therefore, computational complexity of HHO is $\mathrm{O}(N \times(T+T D+1))$.

\section{Experimental resı its an' discussions}

\subsection{Benchmark set a d r ompared algorithms}

In order to inves Igate the efficacy of the proposed HHO optimizer, a well-studied set of diverse benchmark function are s lected from literature $[49,50]$. This benchmark set covers three main groups of bench nark landscapes: unimodal (UM), multimodal (MM), and composition (CM). The UM functi ins (F. - F7) with unique global best can reveal the exploitative (intensification) capacities of difte. nt optimizers, while the MM functions (F8-F23) can disclose the exploration (diversificatı $\eta$ ) ... LO avoidance potentials of algorithms. The mathematical formulation and characteristics $: \mathrm{UM}$ and MM problems are shown in Tables 16, 17, and 18 in Appendix A. The third group probıems (F24-F29) are selected from IEEE CEC 2005 competition [51] and covers hybrid composite, rotated and shifted MM test cases. These CM cases are also utilized in many papers and can expose the performance of utilized optimizers in well balancing the exploration 
and exploitation inclinations and escaping from LO in dealing with challenging problems. Details of the CM test problems are also reported in Table 19 in Appendix A. Figure 8 demonstrates three of composition test problems.

The results and performance of the proposed HHO is compared with other v. all-established optimization techniques such as the GA [22], BBO [22], DE [22], PSO [`.2], JS [34], TLBO [29], BA/BAT [52], FPA [53], FA [54], GWO [55], and MFO [56] algorithms be d on the best, worst, standard deviation (STD) and average of the results (AVG). These algon. ' $\mathrm{ms}$ cover both recently proposed techniques such as MFO, GWO, CS, TLBO, BAT, FPA, a ^d . TA and also, relatively the most utilized optimizers in the field like the GA, DE, PSO, and B.' ‘lgorıthms.

As recommended by Derrac et al. [57], the non-parametric Wiln $x o n$ _ªtistical test with $5 \%$ degree of significance is also performed along with experimental as sessme ts to detect the significant differences between the attained results of different techniques.

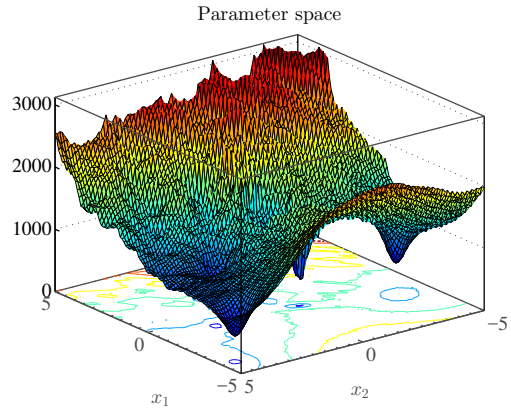

(a) F26

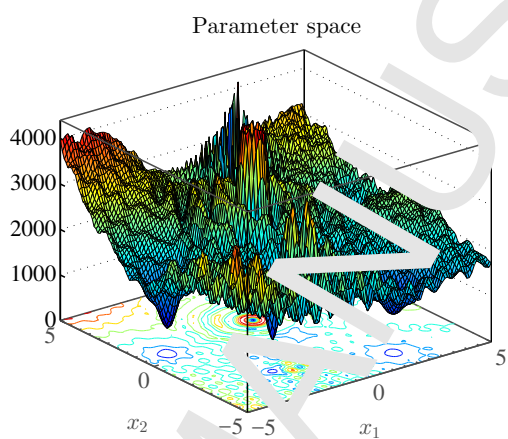

$(\llcorner)$ เ 28

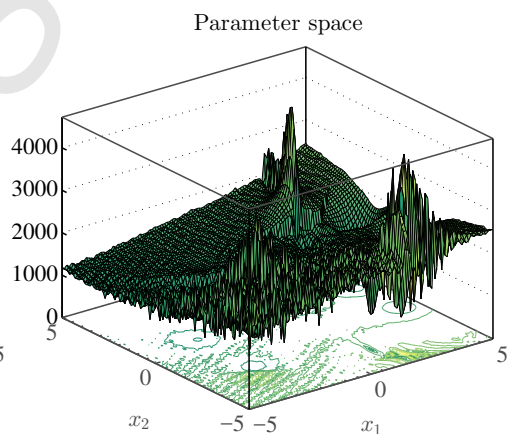

(c) F29

Figure 8: Demonstratioı of composition test functions

\subsection{Experimental setup}

All algorithms were implemente 1 unde Matlab 7.10 (R2010a) on a computer with a Windows 7 64-bit professional and 64 GB I AN. T'e swarm size and maximum iterations of all optimizers are set to 30 and 500, respectiv sly. $\Sigma_{\perp}{ }^{\prime \prime}$ iesults are recorded and compared based on the average performance of optimizers ove $u^{\prime}$ independent runs.

The settings of GA, PSC DE anu BBO algorithms are same with those set by Dan Simon in the original work of BBO 22]. while for the BA [52], FA [58], TLBO [29], GWO [55], FPA [53], CS [34], and MFO [56], the - rameters are same with the recommended settings in the original works. The used param ster, are also reported in Table 1.

\subsection{Qualitative $r$ alts $i$ HHO}

The qualitative esults of HHO for several standard unimodal and multimodal test problems are demonstrated in $1:$ - $y$-11. These results include four well-known metrics: search history, the trajectory of th first awk, average fitness of population, and convergence behavior. In addition, the escaping ent oy of the rabbit is also monitored during iterations. The search history diagram

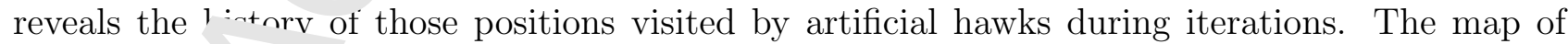
the trajectory $c$. the first hawk monitors how the first variable of the first hawk varies during the steps of the rocess. The average fitness of hawks monitors how the average fitness of whole population varies during the process of optimization. The convergence metric also reveals how the fitness value of the rabbit (best solution) varies during the optimization. Note that the diagram of escaping energy demonstrates how the energy of rabbit varies during the simulation. 

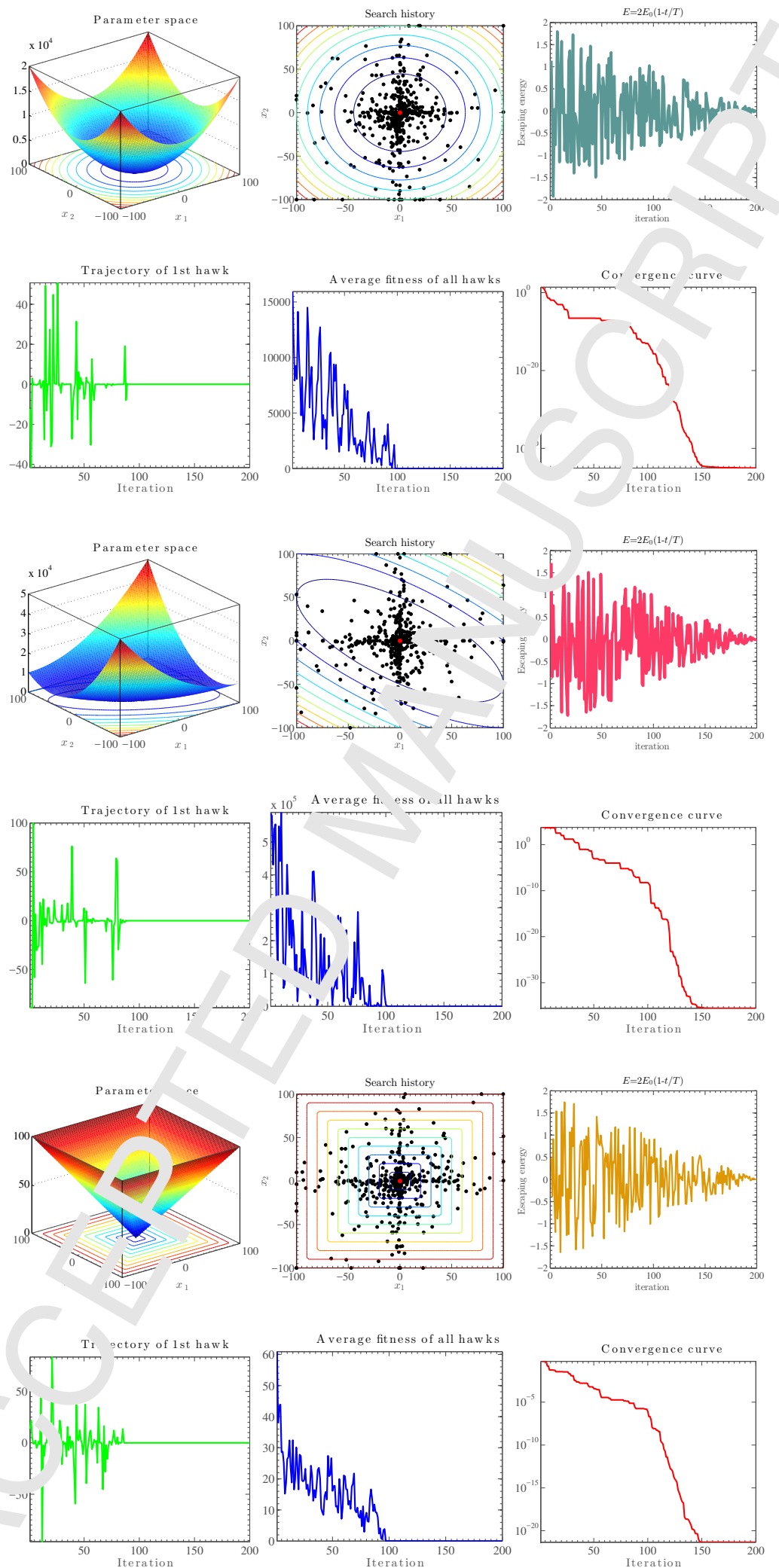

Figure 9: Qualitative results for unimodal F1, F3, and F4 problems 

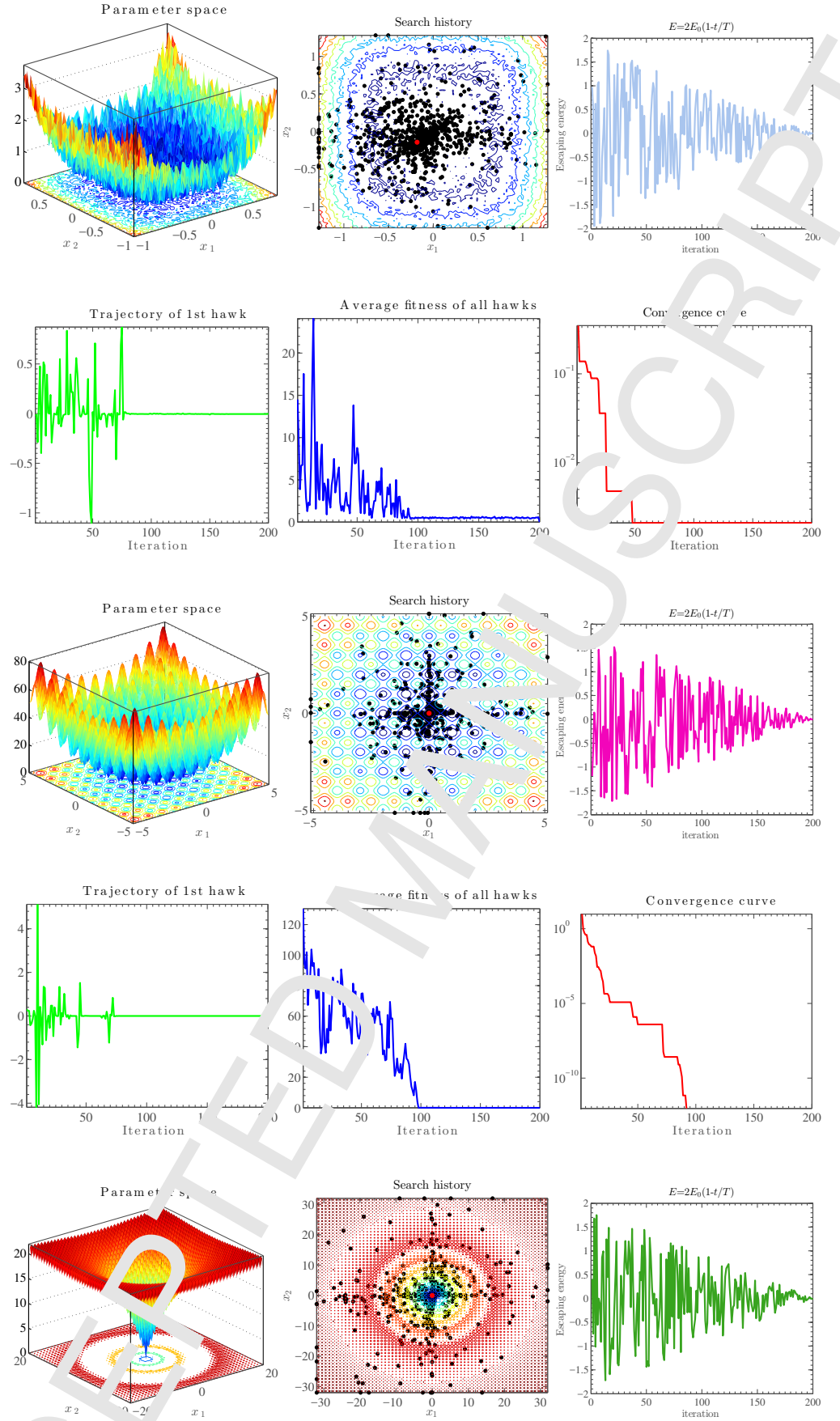

Tra ctory of 1 st hawk
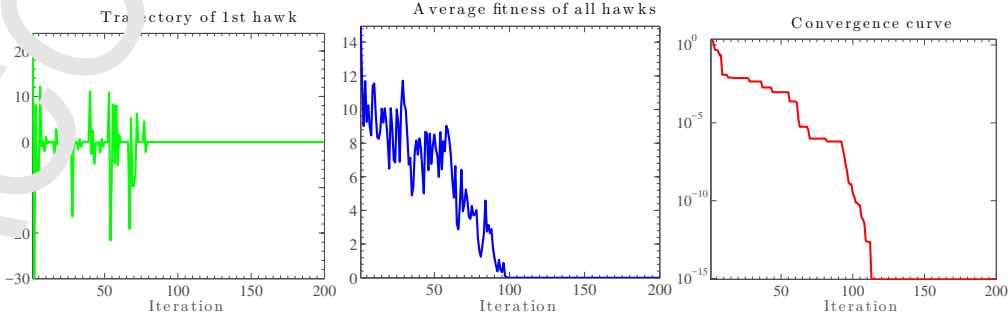

Figure 10: Qualitative results for F7, F9, and F10 problems 
Table 1: The parameter settings

\begin{tabular}{|c|c|c|}
\hline Algorithm & Parameter & Valv \\
\hline \multirow[t]{2}{*}{$\mathrm{DE}$} & Scaling factor & 0.5 \\
\hline & Crossover probability & 0.5 \\
\hline \multirow[t]{4}{*}{ PSO } & Topology fully connected & \\
\hline & Inertia factor & \\
\hline & $c_{1}$ & 1 \\
\hline & $c_{2}$ & 1 \\
\hline TLBO & Teaching factor $T$ & $1,-$ \\
\hline GWO & Convergence constant $a$ & $0]$ \\
\hline MFO & $\begin{array}{l}\text { Convergence constant } a \\
\text { Spiral factor } b\end{array}$ & {$[-1]$} \\
\hline $\mathrm{CS}$ & Discovery rate of alien solutions $p_{a}$ & 0.25 \\
\hline \multirow[t]{4}{*}{$\mathrm{BA}$} & $Q_{\min }$ Frequency minimum & 0 \\
\hline & $Q_{\max }$ Frequency maximum & 2 \\
\hline & $A$ Loudness & 0.5 \\
\hline & $r$ Pulse rate & 0.5 \\
\hline \multirow[t]{3}{*}{ FA } & $\alpha$ & 0.5 \\
\hline & $\beta$ & 0.2 \\
\hline & $\gamma$ & 1 \\
\hline FPA & Probability switch $p$ & 0.8 \\
\hline \multirow[t]{5}{*}{$\mathrm{BBO}$} & Habitat modification probabiı.. & 1 \\
\hline & Immigration probabı ty $11 \ldots$ & {$[0,1]$} \\
\hline & Step size & 1 \\
\hline & Max immigration. ' and 'Tax emigration (E) & 1 \\
\hline & Mutation probabilı! $\quad$ & 0.005 \\
\hline
\end{tabular}
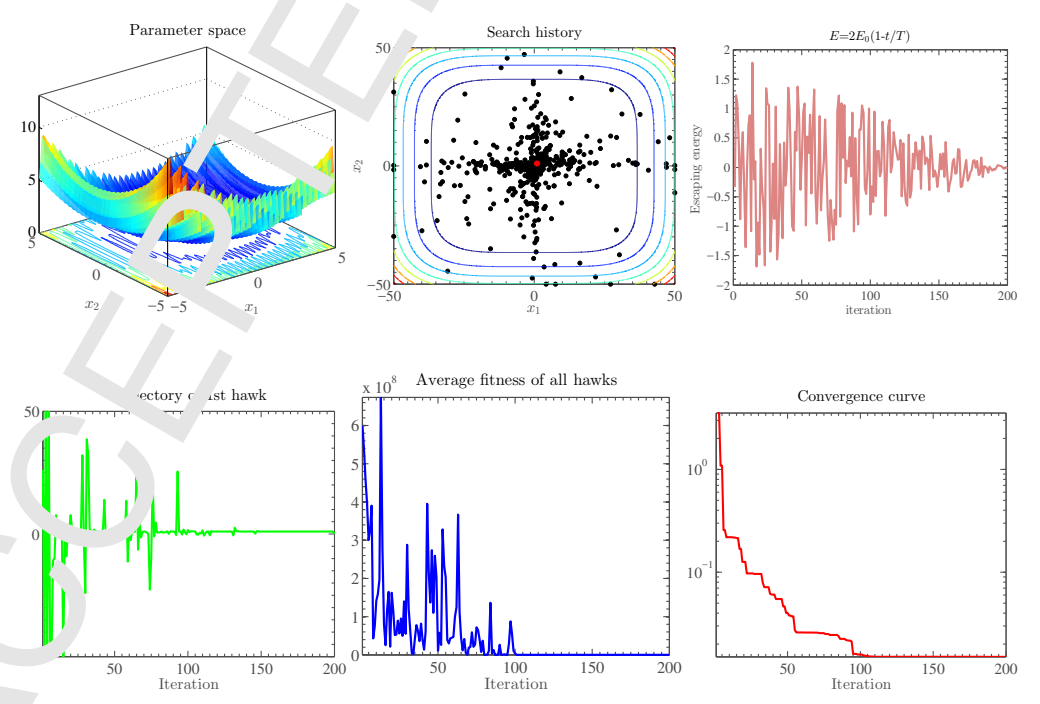

Figure 11: Qualitative results for F13 problem 
From the history of sampled locations in Figs. 9-11, it can be observed that the HHO reveals a similar pattern in dealing with different cases, in which the hawks attempts $t$, initially boost the diversification and explore the favorable areas of solution space and then $\epsilon_{s_{1}}$ loit the vicinity of the best locations. The diagram of trajectories can help us to comprehend the seat hing behavior of the foremost hawk (as a representative of the rest of hawks). By th $\mathrm{s}$ I etric, we can check if the foremost hawk faces abrupt changes during the early phases and $y_{-}$iual variations in the concluding steps. Referring to Van Den Bergh and Engelbrecht [59], thes actrvities can guarantee that a P-metaheuristic finally convergences to a position and exploj to ? target region.

As per trajectories in Figs. 9-11, we see that the foremost haw'. atr it the searching procedure with sudden movements. The amplitude of these variations cover mor than $50 \%$ of the solution space. This observation can disclose the exploration propensiti s of th proposed HHO. As times passes, the amplitude of these fluctuations gradually decreases. This point guarantees the transition of HHO from exploratory trends to exploitative ster s. F-nntually, the motion pattern of the first hawk becomes very stable which shows that the $\mathrm{Hl}^{-}{ }^{-}$is e ploiting the promising regions during the concluding steps. By monitoring the average to ass ut the population, the next measure, we can notice the reduction patterns in fitness va'res whe I the HHO enriches the excellence of the randomized candidate hawks. Based on the dia ${ }^{\text {ram }}{ }^{2}$ monstrated in Figs. 9-11, the HHO can enhance the quality of all hawks during half of the 'ºrations and there is an accelerating decreasing pattern in all curves. Again, the amplituc of variations of fitness results decreases by more iteration. Hence, the HHO can dynamicall! fnons on more promising areas during iterations. According to convergence curves in Fig. Figs. 9-1. which shows the average fitness of best hawk found so far, we can detect accelerated decre ${ }^{\prime} \mathrm{n}$ \& ttterns in all curves, especially after half of the iteration. We can also detect the estimated nonent that the HHO shift from exploration to exploitation. In this regard, it is observed hav ue HHO can reveal an accelerated convergence trend.

\subsection{Scalability analysis}

In this section, a scalability asse smen ${ }^{\circ}$ utilized to investigate the impact of dimension on the results of HHO. This test has bee ut 'izer' in the previous studies and it can reveal the impact of dimensions on the quality of sol tion $f_{C}$. the HHO optimizer to recognize its efficacy not only for problems with lower dimensior s' 'וt also for higher dimension tasks. In addition, it reveals how a P-metaheuristic can preserve its seaı hing advantages in higher dimensions. For this experiment, the HHO is utilized to tacl e $\mathrm{t}$ e scalable UM and MM F1-F13 test cases with 30, 100, 500, and 1000 dimensions. The averac error AVG and STD of the attained results of all optimizers over 30 independent runs an 15 r 0 iterations are recorded and compared for each dimension. Table 2 reveals the results of $\mathrm{H}_{-}{ }^{-T} \mathrm{C}$ ver sus other methods in dealing with F1-F13 problems with different dimensions. The sca' wility s sults for all techniques are also illustrated in Fig. 12. Note that the detailed results of $\varepsilon$ l techi iques are reported in the next parts.

As it can be seeı in iable 2, the HHO can expose excellent results in all dimensions and its performance remain's consistently superior when realizing cases with many variables. As per curves in Fig. 12, it $\mathrm{s}$ observed that the optimality of results and the performance of other methods sig : firantly degrade by increasing the dimensions. This reveals that HHO is capable of maintainin $_{\varepsilon}$ ' good balance between the exploratory and exploitative tendencies on problems with many varia les. 


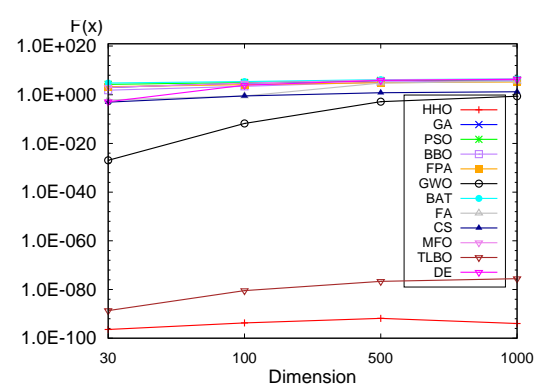

(a) F1

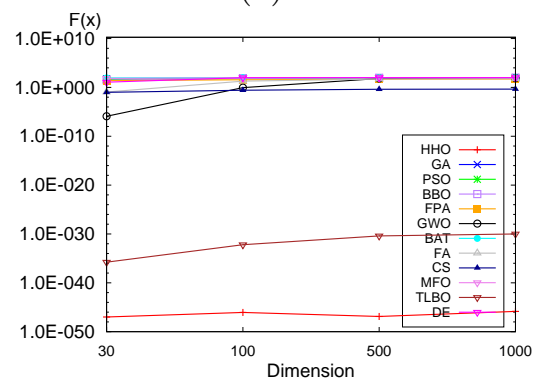

(d) F4

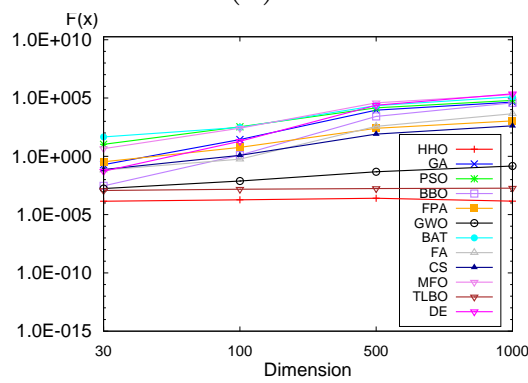

(g) F7

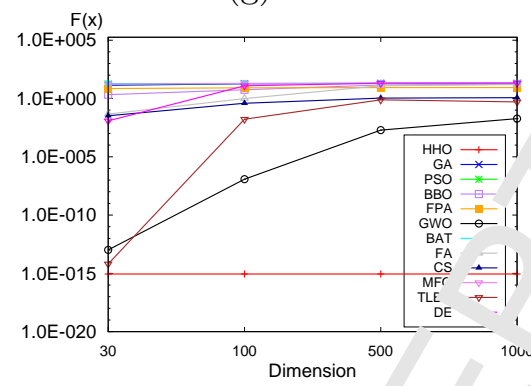

(j) F10

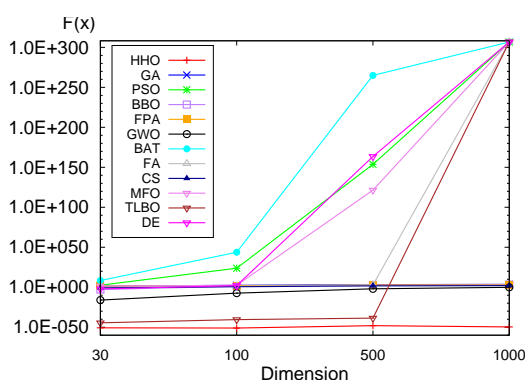

(b) F2

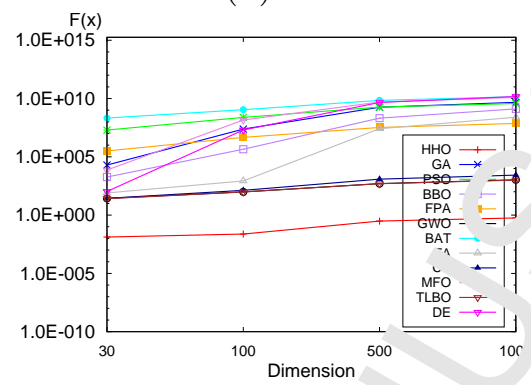

(e) F5

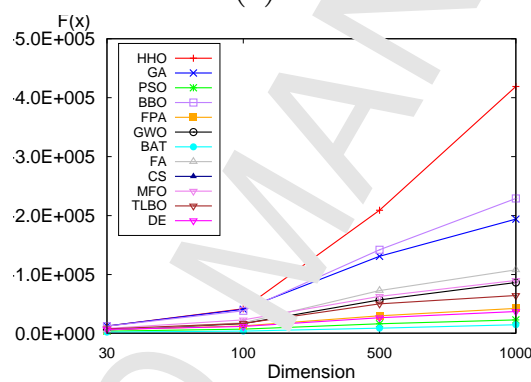

(h) F8

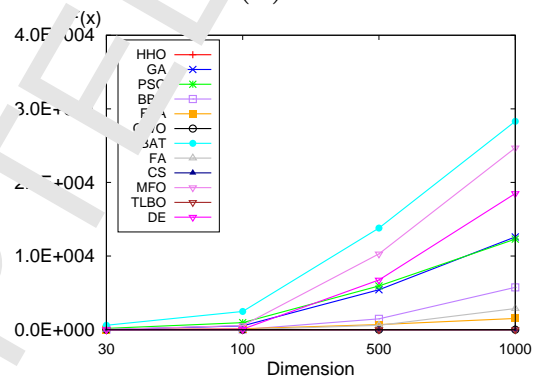

(k) F11

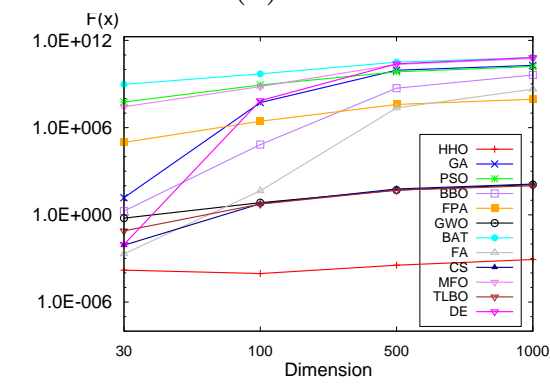

(m) F13

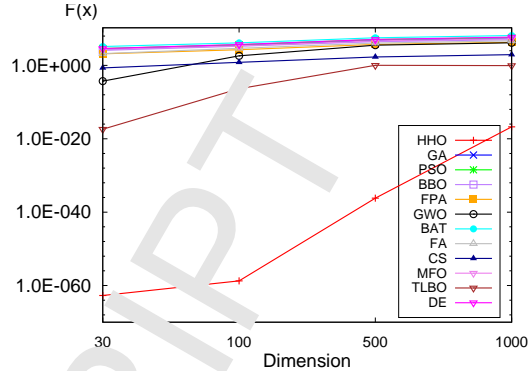

(c) F3

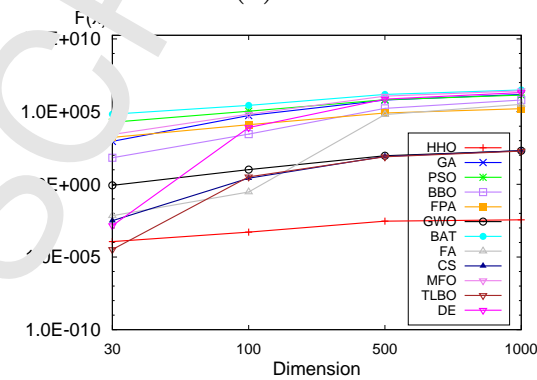

(f) F6

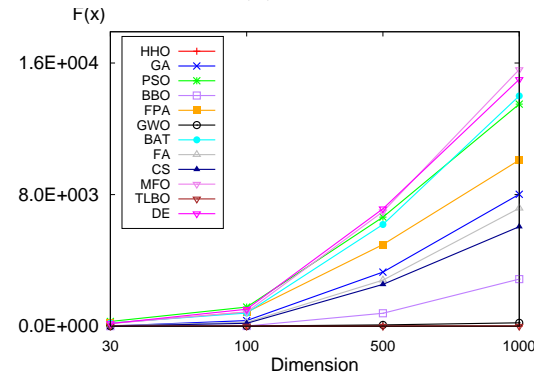

(i) F9

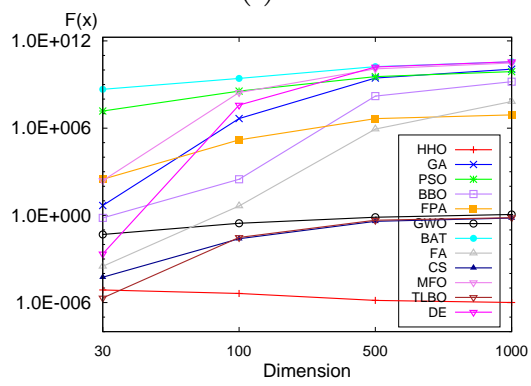

(l) F12

(II) $\mathrm{F} 13$

Figure 12: Scalability results of the HHO versus other methods in dealing with the F1-F13 cases with different dimensions 
Table 2: Results of HHO for different dimensions of scalable F1-F13 problems

\begin{tabular}{|c|c|c|c|c|c|}
\hline Problem/D & Metric & 30 & 100 & 500 & 1000 \\
\hline \multirow{2}{*}{ F1 } & AVG & $3.95 \mathrm{E}-97$ & $1.91 \mathrm{E}-94$ & $1.46 \mathrm{E}-92$ & $1.06 \mathrm{E}-94$ \\
\hline & STD & $1.72 \mathrm{E}-96$ & 8.66E-94 & 8.01E-92 & $4.97 \mathrm{E}-94$ \\
\hline \multirow{2}{*}{$\mathrm{F} 2$} & AVG & $1.56 \mathrm{E}-51$ & $9.98 \mathrm{E}-52$ & $7.87 \mathrm{E}-49$ & $2.52 \mathrm{E}-50$ \\
\hline & STD & $6.98 \mathrm{E}-51$ & $2.66 \mathrm{E}-51$ & $3.11 \mathrm{E}-48$ & $5.02 \mathrm{E}-50$ \\
\hline \multirow{2}{*}{ F3 } & AVG & $1.92 \mathrm{E}-63$ & $1.84 \mathrm{E}-59$ & $6.54 \mathrm{E}-37$ & $1.79 \mathrm{E}-17$ \\
\hline & STD & 1.05E-62 & $1.01 \mathrm{E}-58$ & $3.58 \mathrm{E}-36$ & $9.81 \mathrm{E}-17$ \\
\hline \multirow{2}{*}{$\mathrm{F} 4$} & AVG & $1.02 \mathrm{E}-47$ & $8.76 \mathrm{E}-47$ & $1.29 \mathrm{E}-47$ & $1.43 \mathrm{E}-46$ \\
\hline & STD & $5.01 \mathrm{E}-47$ & $4.79 \mathrm{E}-46$ & $4.11 \mathrm{E}-47$ & $7.74 \mathrm{E}-18$ \\
\hline \multirow{2}{*}{ F5 } & AVG & $1.32 \mathrm{E}-02$ & $2.36 \mathrm{E}-02$ & $3.10 \mathrm{E}-01$ & $\overline{5.73},-\overline{01}$ \\
\hline & STD & $1.87 \mathrm{E}-02$ & $2.99 \mathrm{E}-02$ & $3.73 \mathrm{E}-01$ & 1. $\angle \mathrm{E}+\mathrm{O}^{\mathrm{r}}$ \\
\hline \multirow{2}{*}{ F6 } & AVG & $1.15 \mathrm{E}-04$ & $5.12 \mathrm{E}-04$ & $2.94 \mathrm{E}-03$ & $3.61 \mathrm{~L}=$ \\
\hline & STD & $1.56 \mathrm{E}-04$ & $6.77 \mathrm{E}-04$ & $3.98 \mathrm{E}-03$ & 3E-03 \\
\hline \multirow{2}{*}{ F7 } & AVG & $1.40 \mathrm{E}-04$ & $1.85 \mathrm{E}-04$ & $2.51 \mathrm{E}-04$ & $\overline{1.41 \mathrm{E}-\mathrm{c}}$ \\
\hline & STD & $1.07 \mathrm{E}-04$ & $4.06 \mathrm{E}-04$ & $2.43 \mathrm{E}-04$ & $1.63 \mathrm{E}-04$ \\
\hline \multirow{2}{*}{ F8 } & AVG & $-1.25 \mathrm{E}+04$ & $-4.19 \mathrm{E}+04$ & $-2.09 \mathrm{E}+05$ & $\overline{119 \mathrm{~F}}$ J5 \\
\hline & STD & $1.47 \mathrm{E}+02$ & $2.82 \mathrm{E}+00$ & $2.84 \mathrm{~F},-\mathrm{U}_{\perp}$ & 1.03E+02 \\
\hline \multirow{2}{*}{ F9 } & AVG & $0.00 \mathrm{E}+00$ & $0.00 \mathrm{E}+00$ & $0.00 \overline{+00}$ & $0 .{ }^{\mathrm{TE}+00}$ \\
\hline & STD & $0.00 \mathrm{E}+00$ & $0.00 \mathrm{E}+00$ & $0.00 \mathrm{~L} \quad J$ & $0 . \mathrm{J}^{\mathrm{E}}+00$ \\
\hline \multirow{2}{*}{ F10 } & AVG & $8.88 \mathrm{E}-16$ & $8.88 \mathrm{E}-16$ & $\overline{8} \cdot{ }^{\mathrm{T}}-16$ & $.8 \overline{8 \mathrm{E}-16}$ \\
\hline & STD & $4.01 \mathrm{E}-31$ & $4.01 \mathrm{E}-31$ & $4.01 \mathrm{E}-\mathrm{c}$ & $4.01 \mathrm{E}-31$ \\
\hline \multirow{2}{*}{ F11 } & AVG & $0.00 \mathrm{E}+00$ & $0.00 \mathrm{E}+00$ & $0.00 \mathrm{E}+0 \mathrm{C}$ & $0.00 \mathrm{E}+00$ \\
\hline & STD & $0.00 \mathrm{E}+00$ & $0.00 \mathrm{E}+\mathrm{\cap} 0$ & c. $\cdot \mathrm{E}+\mathrm{f}$ & $0.00 \mathrm{E}+00$ \\
\hline \multirow{2}{*}{ F12 } & AVG & $7.35 \mathrm{E}-06$ & $4.23 \mathrm{E}-06$ & $1.41 \mathrm{E}-\mathrm{U} 6$ & $1.02 \mathrm{E}-06$ \\
\hline & STD & $1.19 \mathrm{E}-05$ & $5.25 \mathrm{E}-06$ & $1.2{ }^{\top}-06$ & $1.16 \mathrm{E}-06$ \\
\hline \multirow{2}{*}{ F13 } & AVG & $1.57 \mathrm{E}-04$ & כיוי ביט & $3.44 \mathrm{E}-04$ & $8.41 \mathrm{E}-04$ \\
\hline & STD & $2.15 \mathrm{E}-04$ & $1.26 \mathrm{E}-\mathrm{O}_{4}$ & $4.75 \mathrm{E}-04$ & $1.18 \mathrm{E}-03$ \\
\hline
\end{tabular}

\subsection{Quantitative results of HHO and "“cus ion}

In this section, the results of $\mathrm{HHO}$ are $\mathrm{com}_{\mathrm{t}}$ are $\mathrm{l}$ with those of other optimizers for different dimensions of F1-F13 test problems in aan tun to F14-F29 MM and CM test cases. Note that the results are presented for 30,100, 500, a.id 1000 dimensions of the scalable F1-F13 problems. Tables 3-6 show the obtained $r_{\text {sut.'s }}$ for HHO versus other competitors in dealing with scalable functions. Table 8 also reve 'ls the serformance of algorithms in dealing with F14-F29 test problems. In order to investig te thr -gnificant differences between the results of proposed $\mathrm{HHO}$ versus other optimizers, $\mathrm{Wi}^{\prime} \mathrm{ox} \cap \mathrm{n}$ r $\_$kk-sum test with $5 \%$ degree is carefully performed here [57]. Tables 20,21, 22, 23, and 24 ? ppendix B show the attained p-values of the Wilcoxon rank-sum test with $5 \%$ signific .. $^{-e}$.

As per result in Table 3, the Hh` can obtain the best results compared to other competitors on F1-F5, F7, and F9-F1? pr blems. The results of HHO are considerably better than other algorithms in dealing with $\_1 \%$ of these 30-dimensional functions, demonstrating the superior performance of this opti niz^r. necording to p-values in Table 20, it is detected that the observed differences in the resul a e st ttistically meaningful for all cases. From Table 4, when we have a 100-dimensional sear -. spa the HHO can considerably outperform other techniques and attain the best results for $92.3 \%$ of F1-F13 problems. It is observed that the results of HHO are again remarkably better th. $n n^{+}$.er techniques. With regard to p-values in Table 21, it is detected that the solutions of $\mathrm{HHC}$ are significantly better than those realized by other techniques in almost all cases. From Table ;, we see that the HHO can attain the best results in terms of AVG and STD in dealino witı 12 test cases with 500 dimensions. By considering p-values in Table 22, it is recognized th, t, ne $\mathrm{HHO}$ can significantly outperform other optimizers in all cases. As per results in Table 6, sim. arly to what we observed in lower dimensions, it is detected that the HHO has still a remarkably superior performance in dealing with F1-F13 test functions compared to GA, PSO, DE, BBO, CS, GWO, MFO, TLBO, BAT, FA, and FPA optimizers. The statistical results in Table 23 also verify the significant gap between the results of HHO and other optimizers in 
almost all cases. It is seen that the HHO has reached the best global optimum for F9 and F11 cases in any dimension.

Table 3: Results of benchmark functions (F1-F13), with 30 dimensıons.

\begin{tabular}{|c|c|c|c|c|c|c|c|c|c|c|c|c|c|}
\hline \multicolumn{2}{|c|}{ Benchmark } & $\mathrm{HHO}$ & GA & PSO & BBO & FPA & GWO & BAT & $\mathrm{FA}$ & CS & MFC & T $\overline{B O}$ & $\mathrm{DE}$ \\
\hline \multirow{2}{*}{$\mathrm{F} 1$} & AVG & $3.95 \mathrm{E}-97$ & $1.03 \mathrm{E}+03$ & $1.83 \mathrm{E}+04$ & $7.59 \mathrm{E}+01$ & $2.01 \mathrm{E}+03$ & $1.18 \mathrm{E}-27$ & $6.59 \mathrm{E}+04$ & $7.11 \mathrm{E}-03$ & $9.06 \mathrm{E}-04$ & $1.0 \pm \overline{3}$ & $.1 \overline{7 \mathrm{E}-89}$ & $1.33 \mathrm{E}-03$ \\
\hline & & & & $3.01 \mathrm{E}+03$ & $2.75 \mathrm{E}+01$ & & & $7.51 \mathrm{E}+03$ & $3.21 \mathrm{E}-03$ & $4.55 \mathrm{E}-04$ & & & \\
\hline \multirow{2}{*}{$\mathrm{F} 2$} & AVG & $1.56 \mathrm{E}-51$ & $2.47 \mathrm{E}+01$ & $3.58 \mathrm{E}+02$ & $1.36 \mathrm{E}-03$ & $3.22 \mathrm{E}+01$ & $9.71 \mathrm{E}-17$ & $2.71 \mathrm{E}+08$ & $4.34 \mathrm{E}-01$ & $1.49 \mathrm{E}-01$ & З.. $\triangle 01$ & 2. 45 & $6.83 \mathrm{E}-03$ \\
\hline & STD & $6.98 \mathrm{E}-51$ & $5.68 \mathrm{E}+00$ & $1.35 \mathrm{E}+03$ & $7.45 \mathrm{E}-03$ & $5.55 \mathrm{E}+00$ & $5.60 \mathrm{E}-17$ & $1.30 \mathrm{E}+09$ & $1.84 \mathrm{E}-01$ & $2.79 \mathrm{E}-02$ & $2.06 \mathrm{E}_{\top}$ & $3.11 \mathrm{E}-45$ & $2.06 \mathrm{E}-03$ \\
\hline \multirow{2}{*}{ F3 } & $\mathrm{AV}$ & & & & & & & & & & $3 \mathrm{E}+04$ & $1 \mathrm{E}-18$ & +04 \\
\hline & STD & $1.05 \mathrm{E}-62$ & $3.44 \mathrm{E}+03$ & $8.21 \mathrm{E}+03$ & $2.69 \mathrm{E}-$ & $5.59 \mathrm{E}+02$ & 2.03E-04 & 4.72E+04 & $6.72 \mathrm{E}+02$ & $5.69 \mathrm{E}-1$ & & $8.04 \mathrm{E}-18$ & $5.37 \mathrm{E}+03$ \\
\hline \multirow{2}{*}{$\mathrm{F} 4$} & AVG & $1.02 \mathrm{E}-47$ & $5.17 \mathrm{E}+01$ & $4.39 \mathrm{E}+01$ & $3.02 \mathrm{E}+01$ & $2.38 \mathrm{E}+01$ & $1.24 \mathrm{E}-06$ & $8.51 \mathrm{E}+01$ & $1.11 \mathrm{E}-01$ & $9.65 \mathrm{E}$ & $\overline{7}, \widehat{\mathrm{E}+01}$ & $1.68 \mathrm{E}-36$ & $1.15 \mathrm{E}+01$ \\
\hline & STD & $5.01 \mathrm{E}-4$ & $05 \mathrm{E}+01$ & $E+00$ & $4.39 \mathrm{E}+00$ & $E+00$ & $1.94 \mathrm{E}-06$ & $2.95 \mathrm{E}+00$ & $4.75 \mathrm{E}-02$ & $1.94 \mathrm{E}-\mathrm{U}_{-}$ & $.06 \mathrm{E}+00$ & $1.47 \mathrm{E}-36$ & $2.37 \mathrm{E}+00$ \\
\hline \multirow{2}{*}{ F5 } & AVG & & & & & & & & 01 & $2.76 \mathrm{~F}+01$ & $-F+03$ & & \\
\hline & STD & $1.87 \mathrm{E}-02$ & $1.31 \mathrm{E}+04$ & $6.25 \mathrm{E}+06$ & $9.40 \mathrm{E}+02$ & $1.75 \mathrm{E}+05$ & $7.78 \mathrm{E}-01$ & $4.17 \mathrm{E}+07$ & $7.39 \mathrm{E}+01$ &, $1 \mathrm{E}-11$ & $2.26 \mathrm{~L}\ulcorner 04$ & $4.26 \mathrm{E}-01$ & $1.01 \mathrm{E}+02$ \\
\hline \multirow{2}{*}{ F6 } & AVG & $15 \mathrm{E}-04$ & $01 \mathrm{E}+02$ & $7 \mathrm{E}+04$ & $71 \mathrm{E}+01$ & $0 \mathrm{E}+03$ & $8.44 \mathrm{E}-01$ & $6.69 \mathrm{E}+04$ & $6.94 \mathrm{E}-03$ & $3.13 \mathrm{E}-03$ & $68 \mathrm{E}+03$ & $3.29 \mathrm{E}-05$ & $1.44 \mathrm{E}-03$ \\
\hline & STD & $1.56 \mathrm{E}-04$ & $2.84 \mathrm{E}+02$ & $2.92 \mathrm{E}+03$ & $2.20 \mathrm{E}+01$ & $3.13 \mathrm{E}+02$ & $3.18 \mathrm{E}-01$ & $5.87 \mathrm{E}+03$ & $3.61 \mathrm{E}-03$ & 1. $.30 \mathrm{E}-03$ & $34 \mathrm{E}+03$ & $8.65 \mathrm{E}-05$ & $5.38 \mathrm{E}-04$ \\
\hline \multirow{2}{*}{ F7 } & AVG & & & & & & & & & 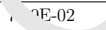 & $.50 \mathrm{E}+00$ & & \\
\hline & STD & $1.07 \mathrm{E}-04$ & $1.50 \mathrm{E}-01$ & $3.05 \mathrm{E}+00$ & $1.83 \mathrm{E}-03$ & $0 \mathrm{E}-01$ & $1.06 \mathrm{E}-03$ & $7.82 \mathrm{E}+00$ & $4.23 \mathrm{E}-r$ & $2.2 \perp_{\perp}$ & $9.21 \mathrm{E}+00$ & 3.63E-04 & $1.37 \mathrm{E}-02$ \\
\hline \multirow{2}{*}{ F8 } & AVG & $-1.25 \mathrm{E}+04$ & $-1.26 \mathrm{E}+04$ & $-3.86 \mathrm{E}+03$ & $-1.24 \mathrm{E}+04$ & $.45 \mathrm{E}+03$ & $-5.97 \mathrm{E}+03$ & $-2.33 \mathrm{E}+03$ & $\overline{-5.5} \Sigma \overline{+03}$ & $-\sim \sim \mathrm{E}+19$ & $-8.48 \mathrm{E}+03$ & $-7.76 \mathrm{E}+03$ & $-6.82 \mathrm{E}+03$ \\
\hline & STD & & & $2.49 \mathrm{E}+02$ & & +02 & $7.10 \mathrm{E}+02$ & $2.96 \mathrm{E}$ & & $.76 \mathrm{E} \quad 20$ & $7.98 \mathrm{E}+02$ & $1.04 \mathrm{E}+03$ & $3.94 \mathrm{I}$ \\
\hline \multirow{2}{*}{ F9 } & AVG & $00 \mathrm{E}+00$ & $9.04 \mathrm{E}+00$ & $2.87 \mathrm{E}+02$ & $0.00 \mathrm{E}+00$ & $1.82 \mathrm{E}+02$ & $2.19 \mathrm{E}+00$ & $1.92 \mathrm{E}+02$ & & $1.51 \mathrm{E} \quad \overline{1}$ & $1.59 \mathrm{E}+02$ & $1.40 \mathrm{E}+01$ & $1.58 \mathrm{E}+02$ \\
\hline & STD & $0.00 \mathrm{E}+00$ & $4.58 \mathrm{E}+00$ & $1.95 \mathrm{E}+01$ & $0.00 \mathrm{E}+00$ & $1.24 \mathrm{E}+01$ & $3.69 \mathrm{E}+00$ & $3.56 \mathrm{E}+01$ & 1. $12 \mathrm{E}+01$ & $1.2^{5}+00$ & $3.21 \mathrm{E}+01$ & $5.45 \mathrm{E}+00$ & $1.17 \mathrm{E}+01$ \\
\hline \multirow{2}{*}{ F10 } & AVG & & & & & & & & & $3.29 \overline{\mathrm{E}-02}$ & $1.74 \mathrm{E}+01$ & & \\
\hline & STD & 4. & & & & & & & $1.20 \mathrm{E}-\mathrm{u}_{\mathrm{L}}$ & 7.93E-03 & $4.95 \mathrm{E}+00$ & $1.79 \mathrm{E}-15$ & E-03 \\
\hline \multirow{2}{*}{ F11 } & AVG & $0.00 \mathrm{E}+00$ & $01 \mathrm{E}+01$ & $1.70 \mathrm{E}+02$ & $1.46 \mathrm{E}+00$ & $1.73 \mathrm{E}+01$ & $4.76 \mathrm{E}-03$ & $6.01 \mathrm{~F} \quad 2$ & $4.23 \mathrm{E}-03$ & $4.29 \mathrm{E}-05$ & $3.10 \mathrm{E}+01$ & $0.00 \mathrm{E}+00$ & $3.52 \mathrm{E}-02$ \\
\hline & STD & $0.00 \mathrm{E}+00$ & $2.43 \mathrm{E}+00$ & $3.17 \mathrm{E}+01$ & $1.69 \mathrm{E}-01$ & $3.63 \mathrm{E}+00$ & $8.57 \mathrm{E}-03$ & $5.50 \mathrm{E}+\mathrm{v}$. & 1.29E-03 & $2.00 \mathrm{E}-05$ & $5.94 \mathrm{E}+01$ & $0.00 \mathrm{E}+00$ & E-02 \\
\hline \multirow{2}{*}{ F12 } & AVG & & & & & & & & & & & & \\
\hline & STD & $1.19 \mathrm{E}-05$ & $1.56 \mathrm{E}+00$ & $9.88 \mathrm{E}+06$ & $2.62 \mathrm{E}-01$ & $1.04 \mathrm{E}+03$ & $2.12 \mathrm{E}-02$ & $1.54 \mathrm{E}+\mathrm{c}$ & $1.76 \mathrm{E}-04$ & $4.96 \mathrm{E}-05$ & $1.21 \mathrm{E}+03$ & $7.45 \mathrm{E}-06$ & $1.70 \mathrm{E}-03$ \\
\hline \multirow{2}{*}{ F13 } & AVG & 04 & & & & & $5.96 \mathrm{E}-01$ & $9.40 \mathrm{E}+08$ & ए-03 & $8.19 \mathrm{E}-03$ & $2.73 \mathrm{E}+07$ & $7.89 \mathrm{E}-02$ & $9.12 \mathrm{E}-03$ \\
\hline & STD & $5 \mathrm{E}-04$ & $4.52 \mathrm{E}+00$ & & E-01 & $1.46 \mathrm{E}+05$ & $2.23 \mathrm{E}-01$ & L.ViLtuo & y. $62 \mathrm{E}-04$ & $6.74 \mathrm{E}-03$ & $1.04 \mathrm{E}+08$ & $8.78 \mathrm{E}-02$ & 1.16E-02 \\
\hline
\end{tabular}

Table 4: Results of benchmark functic.'(F1-F13), with 100 dimensions.

\begin{tabular}{|c|c|c|c|c|c|c|c|c|c|c|c|c|c|}
\hline Ben & nark & $\mathrm{HHO}$ & GA & PSO & BBO & FPA & & $\overline{\mathrm{B}} \mathrm{AT}$ & FA & CS & MFO & TLBO & $\mathrm{DE}$ \\
\hline F1 & $\begin{array}{l}\text { AVG } \\
\text { STD }\end{array}$ & $\begin{array}{l}1.91 \mathrm{E}-94 \\
8.66 \mathrm{E}-94\end{array}$ & $\begin{array}{l}5.41 \mathrm{E}+04 \\
1.42 \mathrm{E}+04\end{array}$ & $\begin{array}{l}1.06 \mathrm{E}+05 \\
8.47 \mathrm{E}+03\end{array}$ & $\begin{array}{l}2.85 \mathrm{E}+03 \\
4.49 \mathrm{E}+02\end{array}$ & $\begin{array}{l}1.39 \mathrm{~F}+04 \\
2.71 \quad \ldots\end{array}$ & $\begin{array}{l}1.59 \mathrm{~L} \\
\mathrm{2}\end{array}$ & $\begin{array}{l}2.72 \mathrm{E}+05 \\
1.42 \mathrm{E}+04\end{array}$ & $\begin{array}{l}3.05 \mathrm{E}-01 \\
5.60 \mathrm{E}-02\end{array}$ & $\begin{array}{l}3.17 \mathrm{E}-01 \\
5.28 \mathrm{E}-02\end{array}$ & $\begin{array}{l}6.20 \mathrm{E}+04 \\
1.25 \mathrm{E}+04\end{array}$ & $\begin{array}{l}3.62 \mathrm{E}-81 \\
4.14 \mathrm{E}-81\end{array}$ & $\begin{array}{l}8.26 \mathrm{E}+03 \\
1.32 \mathrm{E}+03\end{array}$ \\
\hline F2 & $\begin{array}{l}\text { AVG } \\
\text { STD }\end{array}$ & $\begin{array}{l}9.98 \mathrm{E}-52 \\
2.66 \mathrm{E}-51\end{array}$ & $\begin{array}{l}2.53 \mathrm{E}+02 \\
1.41 \mathrm{E}+01\end{array}$ & $\begin{array}{l}6.06 \mathrm{E}+23 \\
2.18 \mathrm{E}+24\end{array}$ & $\begin{array}{l}1.59 \mathrm{E}+01 \\
3.74 \mathrm{E}+00\end{array}$ & $\begin{array}{l}1.01 \mathrm{E}+0< \\
9.36 \mathrm{E}+00\end{array}$ & $\begin{array}{l}\text { 131E- } 08 \\
1 . \quad 08\end{array}$ & $\begin{array}{l}6.00 \mathrm{E}+43 \\
1.18 \mathrm{E}+44\end{array}$ & $\begin{array}{l}1.45 \mathrm{E}+01 \\
6.73 \mathrm{E}+00\end{array}$ & $\begin{array}{l}4.05 \mathrm{E}+00 \\
3.16 \mathrm{E}-01\end{array}$ & $\begin{array}{l}2.46 \mathrm{E}+02 \\
4.48 \mathrm{E}+01\end{array}$ & $\begin{array}{l}3.27 \mathrm{E}-41 \\
2.75 \mathrm{E}-41\end{array}$ & $\begin{array}{l}1.21 \mathrm{E}+02 \\
2.33 \mathrm{E}+01\end{array}$ \\
\hline F3 & $\begin{array}{l}\text { AVG } \\
\text { STD }\end{array}$ & $\begin{array}{l}1.84 \mathrm{E}-59 \\
1.01 \mathrm{E}-58\end{array}$ & $\begin{array}{l}2.53 \mathrm{E}+05 \\
5.03 \mathrm{E}+04\end{array}$ & $\begin{array}{l}4.22 \mathrm{E}+05 \\
7.08 \mathrm{E}+04\end{array}$ & $\begin{array}{l}1.70 \mathrm{E}+05 \\
2.02 \mathrm{E}+0^{4}\end{array}$ & $\begin{array}{r}1.89 \mathrm{E}+04 \\
\mathrm{r}_{+}+03\end{array}$ & $\begin{array}{l}4.09 \mathrm{E}+02 \\
2.77 \mathrm{E}+02\end{array}$ & $\begin{array}{l}1.43 \mathrm{E}+06 \\
6.21 \mathrm{E}+05\end{array}$ & $\begin{array}{l}4.65 \mathrm{E}+04 \\
6.92 \mathrm{E}+03\end{array}$ & $\begin{array}{l}6.88 \mathrm{E}+00 \\
1.02 \mathrm{E}+00\end{array}$ & $\begin{array}{l}2.15 \mathrm{E}+05 \\
4.43 \mathrm{E}+04\end{array}$ & $\begin{array}{l}4.33 \mathrm{E}-07 \\
8.20 \mathrm{E}-07\end{array}$ & $\begin{array}{l}5.01 \mathrm{E}+05 \\
5.87 \mathrm{E}+04\end{array}$ \\
\hline $\mathrm{F} 4$ & $\begin{array}{l}\text { AVG } \\
\text { STD }\end{array}$ & $\begin{array}{l}8.76 \mathrm{E}-47 \\
4.79 \mathrm{E}-46\end{array}$ & & $\begin{array}{l}6.07 \mathrm{E}+01 \\
3.05 \mathrm{E}+00\end{array}$ & $\begin{array}{ll}7.08 \mathrm{E}-1 & 1 \\
4.73 \mathrm{~F}^{-} & 00\end{array}$ & $\begin{array}{l}3.51 \mathrm{E}+ \\
3.37 \mathrm{E}+0 .\end{array}$ & $\begin{array}{l}8.89 \mathrm{E}-01 \\
9.30 \mathrm{E}-01\end{array}$ & & & & & $\begin{array}{l}6.36 \mathrm{E}-33 \\
6.66 \mathrm{E}-33\end{array}$ & $\begin{array}{l}9.62 \mathrm{E}+01 \\
1.00 \mathrm{E}+00\end{array}$ \\
\hline F5 & $\begin{array}{l}\text { AVG } \\
\text { STD }\end{array}$ & $\begin{array}{l}2.36 \mathrm{E}-02 \\
2.99 \mathrm{E}-02\end{array}$ & $\begin{array}{l}2.37 \mathrm{E}+07 \\
8.43 \mathrm{E}+06\end{array}$ & $\begin{array}{l}2.42 \mathrm{E}+08 \\
4.02 \mathrm{E}+07\end{array}$ & $\begin{array}{l}4.47 \mathrm{E}+\mathrm{c} \\
2 \quad 3 \mathrm{E}+05\end{array}$ & $\begin{array}{l}4.64 \mathrm{E}+\mathrm{C} \\
-\mathrm{E}-j\end{array}$ & $\begin{array}{l}9.79 \mathrm{E}+01 \\
6.75 \mathrm{E}-01\end{array}$ & $\begin{array}{l}1.10 \mathrm{E}+09 \\
9.47 \mathrm{E}+07\end{array}$ & $\begin{array}{l}8.46 \mathrm{E}+02 \\
8.13 \mathrm{E}+02\end{array}$ & $\begin{array}{l}1.33 \mathrm{E}+02 \\
7.34 \mathrm{E}+00\end{array}$ & $\begin{array}{l}1.44 \mathrm{E}+08 \\
7.50 \mathrm{E}+07\end{array}$ & $\begin{array}{l}9.67 \mathrm{E}+01 \\
7.77 \mathrm{E}-01\end{array}$ & $\begin{array}{l}1.99 \mathrm{E}+07 \\
5.80 \mathrm{E}+06\end{array}$ \\
\hline F6 & $\begin{array}{l}\text { AVG } \\
\text { STD }\end{array}$ & & & & $\begin{array}{r}.85 \mathrm{E}+{ }^{\prime} \\
4.07 \mathrm{E}-2\end{array}$ & $\begin{array}{l}1.26 \mathrm{E}-\overline{-04} \\
2 .^{\circ} \mathrm{E}+03\end{array}$ & $\begin{array}{l}1.03 \mathrm{E}+01 \\
1.05 \mathrm{E}+00\end{array}$ & & & & & & $\begin{array}{l}8.07 \mathrm{E}+03 \\
1.64 \mathrm{E}+03\end{array}$ \\
\hline F7 & $\begin{array}{l}\text { AVG } \\
\text { STD }\end{array}$ & $\begin{array}{l}.85 \mathrm{E}-04 \\
4.06 \mathrm{E}-04\end{array}$ & $\begin{array}{l}2.73 \mathrm{E}+01 \\
4.45 \mathrm{E}+01\end{array}$ & $\begin{array}{l}3.41 \mathrm{E}+02 \\
8.74 \mathrm{E}-1\end{array}$ & $\begin{array}{cc}5.5 & +00 \\
5.1 & 00\end{array}$ & $\begin{array}{r}5 \overline{\mathrm{E}+00} \\
.16 \mathrm{E}+00\end{array}$ & $\begin{array}{l}7.60 \mathrm{E}-03 \\
2.66 \mathrm{E}-03\end{array}$ & $\begin{array}{l}3.01 \mathrm{E}+02 \\
2.66 \mathrm{E}+01\end{array}$ & $\begin{array}{l}5.65 \mathrm{E}-01 \\
1.64 \mathrm{E}-01\end{array}$ & $\begin{array}{l}1.21 \mathrm{E}+00 \\
2.65 \mathrm{E}-01\end{array}$ & $\begin{array}{l}2.56 \mathrm{E}+02 \\
8.91 \mathrm{E}+01\end{array}$ & $\begin{array}{l}1.50 \mathrm{E}-03 \\
5.39 \mathrm{E}-04\end{array}$ & $\begin{array}{l}1.96 \mathrm{E}+01 \\
5.66 \mathrm{E}+00\end{array}$ \\
\hline F8 & $\begin{array}{l}\text { AVG } \\
\text { STD }\end{array}$ & $\begin{array}{l}-4.19 \mathrm{E}+04 \\
2.82 \mathrm{E}+00\end{array}$ & $\begin{array}{l}-4.10 \mathrm{E}+04 \\
1.14 \mathrm{E}+02\end{array}$ & $\begin{array}{l}-7.3 . \\
4.7\end{array}$ & $\begin{array}{l}-3.85 \mathrm{E}+\mathrm{v} \\
2.80 \mathrm{E}+02\end{array}$ & $\begin{array}{l}1.28 \mathrm{E}+04 \\
4.64 \mathrm{E}+02\end{array}$ & $\begin{array}{l}-1.67 \mathrm{E}+04 \\
2.62 \mathrm{E}+03\end{array}$ & $\begin{array}{l}-4.07 \mathrm{E}+03 \\
9.37 \mathrm{E}+02\end{array}$ & $\begin{array}{l}-1.81 \mathrm{E}+04 \\
3.23 \mathrm{E}+03\end{array}$ & $\begin{array}{l}\mathbf{- 2 . 8 4 \mathrm { E } + 1 8} \\
6.91 \mathrm{E}+18\end{array}$ & $\begin{array}{l}-2.30 \mathrm{E}+04 \\
1.98 \mathrm{E}+03\end{array}$ & $\begin{array}{l}-1.71 \mathrm{E}+04 \\
3.54 \mathrm{E}+03\end{array}$ & $\begin{array}{l}-1.19 \mathrm{E}+04 \\
5.80 \mathrm{E}+02\end{array}$ \\
\hline F9 & $\begin{array}{l}\text { AVG } \\
\text { STD }\end{array}$ & $\begin{array}{l}\mathbf{0 . 0 0 E}+\mathbf{0 0} \\
0.00 \mathrm{E}+00\end{array}$ & $\begin{array}{l}3.39 \mathrm{E}+02 \\
4.17 \mathrm{E}+01\end{array}$ & $\begin{array}{l}.6 \mathrm{E}+03 \\
5.74 \mathrm{E}+01\end{array}$ & $\begin{array}{r}11 \mathrm{E}+00 \\
2.7 \mathrm{r} \quad 00\end{array}$ & $\begin{array}{l}8.47 \mathrm{E}+02 \\
4.01 \mathrm{E}+01\end{array}$ & $\begin{array}{l}1.03 \mathrm{E}+01 \\
9.02 \mathrm{E}+00\end{array}$ & $\begin{array}{l}7.97 \mathrm{E}+02 \\
6.33 \mathrm{E}+01\end{array}$ & $\begin{array}{l}2.36 \mathrm{E}+02 \\
2.63 \mathrm{E}+01\end{array}$ & $\begin{array}{l}1.72 \mathrm{E}+02 \\
9.24 \mathrm{E}+00\end{array}$ & $\begin{array}{l}8.65 \mathrm{E}+02 \\
8.01 \mathrm{E}+01\end{array}$ & $\begin{array}{l}1.02 \mathrm{E}+01 \\
5.57 \mathrm{E}+01\end{array}$ & $\begin{array}{l}1.03 \mathrm{E}+03 \\
4.03 \mathrm{E}+01\end{array}$ \\
\hline F10 & $\begin{array}{l}\text { AVG } \\
\text { STD }\end{array}$ & $\begin{array}{l}8.88 \mathrm{E}-16 \\
4.01 \mathrm{E}-31\end{array}$ & $\begin{array}{l}1.82 \mathrm{E}+01 \\
4.35 \mathrm{E}-0^{1}\end{array}$ & $\begin{array}{ll}1.3 & +01 \\
2.04 & 01\end{array}$ & $\begin{array}{l}5.57 \mathrm{E}+00 \\
4.72 \mathrm{E}-01\end{array}$ & $\begin{array}{l}8.21 \mathrm{E}+00 \\
1.14 \mathrm{E}+00\end{array}$ & $\begin{array}{l}1.20 \mathrm{E}-07 \\
5.07 \mathrm{E}-08\end{array}$ & & & & & $\begin{array}{l}1.66 \mathrm{E}-02 \\
9.10 \mathrm{E}-02\end{array}$ & $\begin{array}{l}1.22 \mathrm{E}+01 \\
8.31 \mathrm{E}-01\end{array}$ \\
\hline F11 & $\begin{array}{l}\text { AVG } \\
\text { STD }\end{array}$ & $\begin{array}{l}0.00 \mathrm{E}+00 \\
0.00 \mathrm{E}+00\end{array}$ & $\begin{array}{l}5.14 \mathrm{E} \\
1.05 \mathrm{E}+02\end{array}$ & 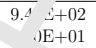 & $\begin{array}{l}2.24 \mathrm{E}+01 \\
4.35 \mathrm{E}+00\end{array}$ & $\begin{array}{l}1.19 \mathrm{E}+02 \\
2.00 \mathrm{E}+01\end{array}$ & $\begin{array}{l}4.87 \mathrm{E}-03 \\
1.07 \mathrm{E}-02\end{array}$ & $\begin{array}{l}2.47 \mathrm{E}+03 \\
1.03 \mathrm{E}+02\end{array}$ & $\begin{array}{l}1.19 \mathrm{E}-01 \\
2.34 \mathrm{E}-02\end{array}$ & $\begin{array}{l}4.56 \mathrm{E}-03 \\
9.73 \mathrm{E}-04\end{array}$ & $\begin{array}{l}5.60 \mathrm{E}+02 \\
1.23 \mathrm{E}+02\end{array}$ & $\begin{array}{l}\mathbf{0 . 0 0 E}+00 \\
0.00 \mathrm{E}+00\end{array}$ & $\begin{array}{l}7.42 \mathrm{E}+01 \\
1.40 \mathrm{E}+01\end{array}$ \\
\hline $\mathrm{F} 12$ & $\begin{array}{l}\text { AVG } \\
\text { STD }\end{array}$ & $\begin{array}{l}4.23 \mathrm{E}-06 \\
5.25 \mathrm{E}-06\end{array}$ & $\left\{\begin{array}{r}4.5+06 \\
2 \mathrm{E}+0 \mathrm{f}\end{array}\right.$ & $\begin{array}{l}3.54 \mathrm{~L} \\
8.75 \mathrm{E}+07\end{array}$ & $\begin{array}{l}3.03 \mathrm{E}+02 \\
1.48 \mathrm{E}+03\end{array}$ & $\begin{array}{l}1.55 \mathrm{E}+05 \\
1.74 \mathrm{E}+05\end{array}$ & $\begin{array}{l}2.87 \mathrm{E}-01 \\
6.41 \mathrm{E}-02\end{array}$ & $\begin{array}{l}2.64 \mathrm{E}+09 \\
2.69 \mathrm{E}+08\end{array}$ & $\begin{array}{l}4.45 \mathrm{E}+00 \\
1.32 \mathrm{E}+00\end{array}$ & $\begin{array}{l}2.47 \mathrm{E}-02 \\
5.98 \mathrm{E}-03\end{array}$ & $\begin{array}{l}2.82 \mathrm{E}+08 \\
1.45 \mathrm{E}+08\end{array}$ & $\begin{array}{l}3.03 \mathrm{E}-02 \\
1.02 \mathrm{E}-02\end{array}$ & $\begin{array}{l}3.90 \mathrm{E}+07 \\
1.88 \mathrm{E}+07\end{array}$ \\
\hline $\mathrm{F} 13$ & $\begin{array}{l}\text { AVG } \\
\text { STD }\end{array}$ & $\begin{array}{l}9.13 \mathrm{E}-05 \\
1.26 \mathrm{E}-04\end{array}$ & & $\begin{array}{l}8.5 \mathrm{f}+08 \\
2 .^{4} \mathrm{E}+08\end{array}$ & & & & & & & & $\begin{array}{l}5.47 \mathrm{E}+00 \\
8.34 \mathrm{E}-01\end{array}$ & $\begin{array}{l}7.19 \mathrm{E}+07 \\
2.73 \mathrm{E}+07\end{array}$ \\
\hline
\end{tabular}

In order to furth r chec the efficacy of HHO, we recorded the running time taken by optimizers to find the solutions $\urcorner_{\imath r}$ T 1 -F13 problems with 1000 dimensions and the results are exposed in Table 7. As per :esult in Table 7, we detect that the HHO shows a reasonably fast and competitive performance in : ndins the best solutions compared to other well-established optimizers even for high dimens inal unimodal and multimodal cases. Based on average running time on 13 problems, the HHO pert ${ }^{r r}$ is faster than BBO, PSO, GA, CS, GWO, and FA algorithms. These observations are also in accor 'ance with the computational complexity of HHO.

The results in Table 8 verify that HHO provides superior and very competitive results on F14-F23 fixed dimension MM test cases. The results on F16-F18 are very competitive and all algorithms have attained high-quality results. Based on results in Table 8, the proposed HHO has 
Table 5: Results of benchmark functions (F1-F13), with 500 dimf to. ns.

\begin{tabular}{|c|c|c|c|c|c|c|c|c|c|c|c|c|c|}
\hline \multicolumn{2}{|c|}{ Benchmark } & $\mathrm{HHO}$ & GA & PSO & $\mathrm{BBO}$ & FPA & GWO & BAT & FA & CS & MFO & $\overline{\mathrm{LBO}}$ & $\mathrm{DE}$ \\
\hline \multirow[t]{2}{*}{ F1 } & AVG & $1.46 \mathrm{E}-92$ & $6.06 \mathrm{E}+05$ & $6.42 \mathrm{E}+05$ & $1.60 \mathrm{E}+05$ & $8.26 \mathrm{E}+04$ & $1.42 \mathrm{E}-03$ & $1.52 \mathrm{E}+06$ & $6.30 \mathrm{E}+04$ & $6.80 \mathrm{E}+00$ & $5 \mathrm{E}+06$ & $\mathrm{~F}_{-}-77$ & $7.43 \mathrm{E}+05$ \\
\hline & & $0.01 \mathrm{~L}$ & 1.01L+07 & $\mathrm{E}+04$ & $9.16 \mathrm{E}+03$ & $2 \mathrm{E}+04$ & $3.99 \mathrm{E}-04$ & $3.58 \mathrm{E}+04$ & $8.47 \mathrm{E}+03$ & $4.93 \mathrm{E}-01$ & $3.54_{2}$ & $1.94 \perp .77$ & $3.67 \mathrm{E}+04$ \\
\hline F2 & $\begin{array}{l}\text { AVG } \\
\text { STD }\end{array}$ & $\begin{array}{l}7.87 \mathrm{E}-49 \\
3.11 \mathrm{E}-48\end{array}$ & $\begin{array}{l}1.94 \mathrm{E}+03 \\
7.03 \mathrm{E}+01\end{array}$ & $\begin{array}{l}6.08 \mathrm{E}+09 \\
1.70 \mathrm{E}+10\end{array}$ & $\begin{array}{l}5.95 \mathrm{E}+02 \\
1.70 \mathrm{E}+01\end{array}$ & $\begin{array}{l}5.13 \mathrm{E}+02 \\
4.84 \mathrm{E}+01\end{array}$ & $\begin{array}{l}1.10 \mathrm{E}-02 \\
1.93 \mathrm{E}-03\end{array}$ & $\begin{array}{l}8.34 \mathrm{E}+09 \\
1.70 \mathrm{E}+10\end{array}$ & $\begin{array}{l}7.13 \mathrm{E}+02 \\
3.76 \mathrm{E}+01\end{array}$ & $\begin{array}{l}4.57 \mathrm{E}+01 \\
2.05 \mathrm{E}+0 \mathrm{r}\end{array}$ & $\begin{array}{r}10 \mathrm{E}+08 \\
\text { 1. } \quad \mathrm{E}+09\end{array}$ & $\begin{array}{r}21 \mathrm{E}-39 \\
1.03 \mathrm{E}-39\end{array}$ & $\begin{array}{l}3.57 \mathrm{E}+09 \\
1.70 \mathrm{E}+10\end{array}$ \\
\hline \multirow{2}{*}{ F3 } & AVG & $6.54 \mathrm{E}-37$ & $5.79 \mathrm{E}+06$ & $1.13 \mathrm{E}+07$ & $2.98 \mathrm{E}+06$ & $5.34 \mathrm{E}+05$ & $3.34 \mathrm{E}+05$ & $3.37 \mathrm{E}+07$ & $1.19 \mathrm{E}+06$ & $\overline{2.03 \mathrm{E}+2} 2$ & & $1.06 \mathrm{E}+00$ & $1.20 \mathrm{E}+07$ \\
\hline & STD & 3. & & $3 \mathrm{E}+06$ & $\mathrm{E}+05$ & $4 \mathrm{E}+05$ & $7.95 \mathrm{E}+04$ & $41 \mathrm{E}+07$ & $3 \mathrm{E}+05$ & & $12 \mathrm{E}+06$ & $3.70 \mathrm{E}+00$ & 1.49E+06 \\
\hline \multirow{2}{*}{$\mathrm{F} 4$} & AVG & $1.29 \mathrm{E}$ & $9.59 \mathrm{E}+01$ & $8.18 \mathrm{E}+01$ & $9.35 \mathrm{E}+01$ & $4.52 \mathrm{E}+01$ & $6.51 \mathrm{E}+01$ & $9.82 \mathrm{E}+01$ & $5.00 \mathrm{E}+01$ & $4.06 \mathrm{E}-01$ & $88 \mathrm{E}+01$ & $4.02 \mathrm{E}-31$ & $9.92 \mathrm{E}+01$ \\
\hline & STD & $4.11 \mathrm{E}-47$ & $1.20 \mathrm{E}+00$ & $1.49 \mathrm{E}+00$ & $9.05 \mathrm{E}-01$ & $4.28 \mathrm{E}+00$ & $5.72 \mathrm{E}+00$ & $3.32 \mathrm{E}-01$ & $1.73 \mathrm{E}+00$ & & 4.1. $\urcorner_{1}$ & $2.67 \mathrm{E}-31$ & $2.33 \mathrm{E}-01$ \\
\hline \multirow{2}{*}{ F5 } & AVG & $3.10 \mathrm{E}-01$ & $1.79 \mathrm{E}+09$ & $1.84 \mathrm{E}+09$ & $2.07 \mathrm{E}+08$ & $3.30 \mathrm{E}+07$ & $4.98 \mathrm{E}+02$ & $6.94 \mathrm{E}+09$ & $2.56 \mathrm{E}+07$ & $.21 \mathrm{E}+03$ & $5.01 \mathrm{E}+09$ & $4.97 \mathrm{E}+02$ & $4.57 \mathrm{E}+09$ \\
\hline & STD & 3.73E-01 & $4.11 \mathrm{E}+08$ & $1.11 \mathrm{E}+08$ & $2.08 \mathrm{E}+07$ & $8.76 \mathrm{E}+06$ & $5.23 \mathrm{E}-01$ & $2.23 \mathrm{E}+08$ & $6.14 \mathrm{E}+06$ & $7.04 \mathrm{E}+01$ & . $50 \mathrm{E}+08$ & $3.07 \mathrm{E}-01$ & \\
\hline \multirow{2}{*}{ F6 } & AVG & $2.94 \mathrm{E}$ & $6.27 \mathrm{E}-$ & 05 & & & $9.22 \mathrm{E}-$ & $1.53 \mathrm{E}+06$ & $6.30 \mathrm{E}+04$ & $7 \mathrm{E}+01$ & $16 \mathrm{E}+06$ & $7.82 \mathrm{E}+01$ & $7.23 \mathrm{E}+05$ \\
\hline & STD & $3.98 \mathrm{E}-03$ & $7.43 \mathrm{E}+04$ & $3.29 \mathrm{E}+04$ & $8.23 \mathrm{E}+03$ & $9.32 \mathrm{E}+03$ & $2.15 \mathrm{E}+00$ & $3.37 \mathrm{E}+04$ & $8.91 \mathrm{E}+03$ & & $.48 \mathrm{E}+04$ & $2.50 \mathrm{E}+00$ & $3.28 \mathrm{E}+04$ \\
\hline \multirow{2}{*}{ F7 } & AVG & $2.51 \mathrm{E}-04$ & $9.10 \mathrm{E}+03$ & $1.43 \mathrm{E}+04$ & $2.62 \mathrm{E}+03$ & $2.53 \mathrm{E}+02$ & $4.67 \mathrm{E}-02$ & $2.23 \mathrm{E}+04$ & $3.71^{\top}-02$ & $8.05 \mathrm{E}+01$ & $3.84 \mathrm{E}+04$ & $1.71 \mathrm{E}-03$ & $2.39 \mathrm{E}+04$ \\
\hline & STD & $2.43 \mathrm{E}-0$ & $2.20 \mathrm{E}+03$ & $1.51 \mathrm{E}+03$ & $3.59 \mathrm{E}+02$ & $6.28 \mathrm{E}+01$ & $1.12 \mathrm{E}-02$ & $1.15 \mathrm{E}+03$ & $6.7+01$ & .. 01 & $2.24 \mathrm{E}+03$ & $4.80 \mathrm{E}-04$ & $2.72 \mathrm{E}+03$ \\
\hline \multirow{2}{*}{ F8 } & AVG & $-2.09 \mathrm{E}+05$ & $-1.31 \mathrm{E}+05$ & $-1.65 \mathrm{E}+04$ & $-1.42 \mathrm{E}+05$ & $-3.00 \mathrm{E}+04$ & $-5.70 \mathrm{E}+04$ & $-9.03 \mathrm{E}+03$ & -7.. $\overline{\mathrm{E}+\mathrm{r}}$ & $-\overline{-2.101}-\overline{-17}$ & $-6.29 \mathrm{E}+04$ & $-5.02 \mathrm{E}+04$ & $-2.67 \mathrm{E}+04$ \\
\hline & STD & $2.84 \mathrm{E}+01$ & $2.31 \mathrm{E}+04$ & $9.99 \mathrm{E}+02$ & $1.98 \mathrm{E}+03$ & $1.14 \mathrm{E}+03$ & $3.12 \mathrm{E}+03$ & $2.12 \mathrm{E}+03$ & $1.15 \mathrm{~b}, \cdots t$ & $1.14 \mathrm{~F} \quad 18$ & $5.71 \mathrm{E}+03$ & $1.00 \mathrm{E}+04$ & $1.38 \mathrm{E}+03$ \\
\hline \multirow{2}{*}{ F9 } & AVG & $00 \mathrm{E}+00$ & $29 \mathrm{E}+03$ & $6.63 \mathrm{E}+$ & $7.86 \mathrm{E}+02$ & $4.96 \mathrm{E}+03$ & $7.84 \mathrm{E}+01$ & $6.18 \mathrm{E}+03$ & $F_{1}+03$ & +03 & $6.96 \mathrm{E}+03$ & +00 & $7.14 \mathrm{E}+03$ \\
\hline & STD & $00 \mathrm{E}+00$ & $1.96 \mathrm{E}+02$ & $7 \mathrm{E}+02$ & $42 \mathrm{E}+01$ & $64 \mathrm{E}+01$ & $3.13 \mathrm{E}+01$ & $20 \mathrm{E}+02$ & $1.42 \mathrm{~L}$ & $5.21 \mathrm{E}+01$ & $1.48 \mathrm{E}+02$ & $0.00 \mathrm{E}+00$ & $1.05 \mathrm{E}+02$ \\
\hline \multirow{2}{*}{ F10 } & AVG & $8.88 \mathrm{E}-16$ & $1.96 \mathrm{E}+01$ & $1.97 \mathrm{E}+01$ & $1.44 \mathrm{E}+01$ & $8.55 \mathrm{E}+00$ & $1.93 \mathrm{E}-03$ & $2.04 \mathrm{E}+01$ & $1.24 \mathrm{E}+01$ & $1.07 \mathrm{E}+00$ & $2.03 \mathrm{E}+01$ & $7.62 \mathrm{E}-01$ & $2.06 \mathrm{E}+01$ \\
\hline & STD & & & 1.04 & & $8.66 \mathrm{~F}$ & & & Q-01 & & $1.48 \mathrm{E}-01$ & $2.33 \mathrm{E}+00$ & E-01 \\
\hline \multirow{2}{*}{ F11 } & AVG & & & & & & & & ${ }^{2}+{ }^{\prime} r$ & & $1.03 \mathrm{E}+04$ & & \\
\hline & STD & $0.00 \mathrm{E}+00$ & $7.32 \mathrm{E}+02$ & $3.19 \mathrm{E}+02$ & $8.10 \mathrm{E}+01$ & $8.17 \mathrm{E}+01$ & $3.50 \mathrm{E}-02$ & $3.19 \mathrm{~L} \quad$ & $7.33 \mathrm{E}+01$ & $2.30 \mathrm{E}-03$ & $4.43 \mathrm{E}+02$ & $0.00 \mathrm{E}+00$ & $2.97 \mathrm{E}+02$ \\
\hline \multirow{2}{*}{ F12 } & AVG & 41E-06 & $2.79 \mathrm{E}$ & & & $4.50 \mathrm{~F}$ & 7.42E-0 & 110 & $7 \mathrm{E}+05$ & $3.87 \mathrm{E}-01$ & $1.20 \mathrm{E}+10$ & 4.61E- -1 & 10 \\
\hline & STD & & & & & & 4.38 & & $\Delta+05$ & $2.47 \mathrm{I}$ & $6.82 \mathrm{E}+08$ & $2.40 \mathrm{E}-02$ & +09 \\
\hline \multirow{2}{*}{ F13 } & AVG & 44E-04 & & & $5.13 \mathrm{E}$ & $3.94 \mathrm{E}$ & $5.06 \mathrm{E}+01$ & ${ }^{7} \mathrm{E}+10$ & $2.29 \mathrm{E}+07$ & & $2.23 \mathrm{E}+10$ & $4.98 \mathrm{E}+01$ & $2.42 \mathrm{E}+10$ \\
\hline & STD & & & & $6.59 \mathrm{E}+07$ & $1.87 \mathrm{E}+07$ & $1.30 \mathrm{E}+00$ & $9.6 \delta_{\perp} \quad x$ & $9.46 \mathrm{E}+06$ & & $1.13 \mathrm{E}+09$ & $9.97 \mathrm{E}-03$ & $6.39 \mathrm{E}+09$ \\
\hline
\end{tabular}

Table 6: Results of $\mathrm{r}$ unch marn functions (F1-F13), with 1000 dimensions.

\begin{tabular}{|c|c|c|c|c|c|c|c|c|c|c|c|c|c|}
\hline \multicolumn{2}{|c|}{ Benchmark } & $\mathrm{HHO}$ & GA & PSO & 1 & $2 \overline{\mathrm{A}}$ & GWO & BAT & FA & CS & MFO & TLBO & $\mathrm{DE}$ \\
\hline \multirow[t]{2}{*}{ F1 } & AVG & 1.06E-94 & $1.36 \mathrm{E}+06$ & 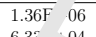 & $6.51 \mathrm{E}_{\mathrm{T}}$ & $1.70 \mathrm{E}+05$ & $2.42 \mathrm{E}-01$ & $3.12 \mathrm{E}+06$ & $3.20 \mathrm{E}+05$ & $1.65 \mathrm{E}+01$ & $2.73 \mathrm{E}+06$ & $2.73 \mathrm{E}-76$ & $2.16 \mathrm{E}+06$ \\
\hline & & & & & $106 \mathrm{~F}$ & $2.991 \mathrm{~K}$ & & & -04 & & $4.10 \mathrm{E}+04$ & & $3.39 \mathrm{E}+00$ \\
\hline $\mathrm{F} 2$ & $\begin{array}{l}\text { AVG } \\
\text { STD }\end{array}$ & $\begin{array}{l}\text { 2.52E-50 } \\
5.02 \mathrm{E}-50\end{array}$ & $\begin{array}{l}4.29 \mathrm{E}+03 \\
8.86 \mathrm{E}+01\end{array}$ & $\begin{array}{l}1.5 \mathrm{E}+1 \mathrm{~L} \\
1.79 \mathrm{E}+10\end{array}$ & $\begin{aligned} 90 E+00 \\
2 . \quad+01\end{aligned}$ & $\begin{array}{l}8.346+02 \\
8.96 \mathrm{E}+01\end{array}$ & $\begin{array}{l}7.11 \mathrm{E}-01 \\
4.96 \mathrm{E}-01\end{array}$ & $1.79 \mathrm{E}+10$ & $\begin{array}{l}.79 \mathrm{E}+10 \\
1.79 \mathrm{E}+10\end{array}$ & $\begin{array}{l}1.02 \mathrm{EE}+02 \\
3.49 \mathrm{E}+00\end{array}$ & $\begin{array}{l}.79 \mathrm{E}+10 \\
1.79 \mathrm{E}+10\end{array}$ & $\begin{array}{l}1.79 \mathrm{E}+10 \\
1.79 \mathrm{E}+10\end{array}$ & $\begin{array}{l}1.79 \mathrm{E}+10 \\
1.79 \mathrm{E}+10\end{array}$ \\
\hline \multirow{2}{*}{ F3 } & AVG & 17 & $29 \mathrm{E}+07$ & $5+07$ & $9.92 \mathrm{E}+06$ & $1.95 \mathrm{E}+06$ & $1.49 \mathrm{E}+06$ & +08 & +06 & & & & +07 \\
\hline & STD & $81 \mathrm{E}-17$ & $3.93 \mathrm{E}+0^{\prime}$ & $1.16 \quad-07$ & $1.48 \mathrm{E}+06$ & $4.20 \mathrm{E}+05$ & $2.43 \mathrm{E}+05$ & $4.76 \mathrm{E}+07$ & $7.19 \mathrm{E}+05$ & +02 & +06 & $1.33 \mathrm{E}+00$ & $14 \mathrm{E}+06$ \\
\hline \multirow{2}{*}{$\mathrm{F} 4$} & $\mathrm{AVG}$ & $1.43 \mathrm{E}-46$ & $9.79 \mathrm{E}-1$ & $\overline{8.9^{\circ}}, \overline{01}$ & $9.73 \mathrm{E}+01$ & $5.03 \mathrm{E}+01$ & $7.94 \mathrm{E}+01$ & $9.89 \mathrm{E}+01$ & $6.06 \mathrm{E}+01$ & $4.44 \mathrm{E}-01$ & $9.96 \mathrm{E}+01$ & $1.01 \mathrm{E}-30$ & $9.95 \mathrm{E}+01$ \\
\hline & STD & 7.74E-46 & $7.16 \mathrm{E}-0 \mathrm{1}$ & $? \quad E+00$ & $7.62 \mathrm{E}-01$ & $5.37 \mathrm{E}+00$ & $2.77 \mathrm{E}+00$ & $2.22 \mathrm{E}-01$ & $2.69 \mathrm{E}+00$ & $2.24 \mathrm{E}-02$ & $1.49 \mathrm{E}-01$ & $5.25 \mathrm{E}-31$ & $1.43 \mathrm{E}-01$ \\
\hline \multirow{2}{*}{ F5 } & AVG & 5.73 & $4.7+09$ & 3.. -09 & $1.29 \mathrm{E}+09$ & $7.27 \mathrm{E}+07$ & -03 & +10 & $2.47 \mathrm{E}+08$ & +03 & & & $8+10$ \\
\hline & STD & $1.40 \mathrm{E}+00$ & $9 \quad 3 \mathrm{E}+08$ & $2.76 \mathrm{E}+.3$ & $6.36 \mathrm{E}+07$ & $1.84 \mathrm{E}+07$ & $3.07 \mathrm{E}+01$ & $3.20 \mathrm{E}+08$ & $3.24 \mathrm{E}+07$ & $1.27 \mathrm{E}+02$ & $3.15 \mathrm{E}+08$ & $2.01 \mathrm{E}-01$ & $3.06 \mathrm{E}+08$ \\
\hline \multirow[b]{2}{*}{ F6 } & AVG & $3.61 \mathrm{E}-03$ &.$\overline{52 \mathrm{E}+r}$ & $1.38^{\Gamma}+06$ & $6.31 \mathrm{E}+05$ & $1.60 \mathrm{E}+05$ & $2.03 \mathrm{E}+02$ & $3.11 \mathrm{E}+06$ & $3.18 \mathrm{E}+05$ & $2.07 \mathrm{E}+02$ & +06 & $1.93 \mathrm{E}+02$ & $2.04 \mathrm{E}+06$ \\
\hline & STD & $5.38 \mathrm{E}-$ & & $6.0+04$ & $1.82 \mathrm{E}+04$ & $1.86 \mathrm{E}+04$ & $2.45 \mathrm{E}+00$ & $6.29 \mathrm{E}+04$ & $2.47 \mathrm{E}$ & $4.12 \mathrm{E}+00$ & $4.56 \mathrm{E}$ & $2.35 \mathrm{E}+00$ & \\
\hline \multirow{2}{*}{ F7 } & $\mathrm{AVC}$ & & $\overline{4} .45 \mathrm{~L}$ & F.$\overline{\mathrm{E}+04}$ & $3.84 \mathrm{E}+04$ & & & & & & & & +05 \\
\hline & STD & $1.63 \mathrm{E}-\mathrm{r}$ & $8.40 \mathrm{E}+0$ & $.16 \mathrm{E}+03$ & $2.91 \mathrm{E}+03$ & $3.49 \mathrm{E}+02$ & $3.28 \mathrm{E}-02$ & $3.93 \mathrm{E}+03$ & $4.00 \mathrm{E}+02$ & $8.22 \mathrm{E}+01$ & $6.19 \mathrm{E}+03$ & $5.79 \mathrm{E}-04$ & $3.52 \mathrm{E}+04$ \\
\hline \multirow{2}{*}{ F8 } & AVG & $-4.19+\overline{05}$ & -1.94 .05 & $-2.30 \mathrm{E}+04$ & $-2.29 \mathrm{E}+05$ & $-4.25 \mathrm{E}+04$ & $-8.64 \mathrm{E}+04$ & $-1.48 \mathrm{E}+04$ & $-1.08 \mathrm{E}+05$ & $-9.34 \mathrm{E}+14$ & $-9.00 \mathrm{E}+04$ & $-6.44 \mathrm{E}+04$ & $-3.72 \mathrm{E}+04$ \\
\hline & STD & & $9.74 \mathrm{E}+3$ & $1.70 \mathrm{E}+03$ & $76 \mathrm{E}+03$ & 1.47 & +04 & & & & & & \\
\hline \multirow{2}{*}{ F9 } & AVG & $0.00 \perp \overline{00}$ & $8.02 \mathrm{E}\lrcorner 3$ & $1.35 \mathrm{E}+04$ & $2.86 \mathrm{E}+03$ & $1.01 \mathrm{E}+04$ & $2.06 \mathrm{E}+02$ & $1.40 \mathrm{E}+04$ & $7.17 \mathrm{E}+03$ & $6.05 \mathrm{E}+03$ & $1.56 \mathrm{E}+04$ & $0.00 \mathrm{E}+00$ & $1.50 \mathrm{E}+04$ \\
\hline & STD & $0.00 \mathrm{E}+\mathrm{l}$ & $2 n 1^{\prime} \quad 02$ & $1.83 \mathrm{E}+02$ & $9.03 \mathrm{E}+01$ & $1.57 \mathrm{E}+02$ & $4.81 \mathrm{E}+01$ & $1.85 \mathrm{E}+02$ & $1.88 \mathrm{E}+02$ & $1.41 \mathrm{E}+02$ & $1.94 \mathrm{E}+02$ & $0.00 \mathrm{E}+00$ & $1.79 \mathrm{E}+02$ \\
\hline \multirow{2}{*}{ F10 } & & & & & $67 \mathrm{E}+$ & & & & & & & & \\
\hline & St & 4. & $2.55 \mathrm{E}-01$ & $1.24 \mathrm{E}-01$ & 8.63E- 02 & $9.10 \mathrm{E}-0$ & $2.74 \mathrm{E}-03$ & 2.23E-02 & $2.42 \mathrm{E}-01$ & $5.90 \mathrm{E}-02$ & $2.16 \mathrm{E}-01$ & $1.94 \mathrm{E}+00$ & $1.06 \mathrm{E}-01$ \\
\hline \multirow{2}{*}{ F11 } & $\overline{\mathrm{AV}}$ & $0.00 \mathrm{E}+00$ & $1.26 \mathrm{E}+04$ & $1.23 \mathrm{E}+04$ & $75 \mathrm{E}+03$ & $52 \mathrm{E}+03$ & $6.58 \mathrm{E}-02$ & +04 & $2.87 \mathrm{E}+$ & & +04 & $1.07 \mathrm{E}-16$ & $1.85 \mathrm{E}+04$ \\
\hline & ST & 0.00 & $1.63 \mathrm{E}+03$ & $\mathrm{E}+02$ & $78 \mathrm{E}+02$ & $2.66 \mathrm{E}+02$ & 8.82 & $21 \mathrm{E}+02$ & $1.78 \mathrm{E}+02$ & 3.58 & $4.51 \mathrm{E}+02$ & $2.03 \mathrm{E}-17$ & $22 \mathrm{E}+03$ \\
\hline \multirow{2}{*}{$\mathrm{F} 1^{\circ}$} & AVG & & & & $1.56 \mathrm{E}$ & & & & & & & & \\
\hline & & 1 16E-06 & & $6.72 \mathrm{E}+08$ & $46 \mathrm{E}+08$ & $3.46 \mathrm{E}+06$ & $1.82 \mathrm{E}-01$ & $1.11 \mathrm{E}+09$ & & & & $1.90 \mathrm{E}-02$ & $7.67 \mathrm{E}+08$ \\
\hline \multirow{2}{*}{ F13 } & $4 \mathrm{~V}$ & & & $1.58 \mathrm{E}+10$ & & & & & & & & & \\
\hline & & & & $1.56 \mathrm{E}+09$ & $2.54 \mathrm{E}+08$ & $3.65 \mathrm{E}+07$ & $1.11 \mathrm{E}+01$ & $1.40 \mathrm{E}+09$ & $7.91 \mathrm{E}+07$ & $1.48 \mathrm{E}+00$ & $1.76 \mathrm{E}+09$ & $1.31 \mathrm{E}-02$ & $2.26 \mathrm{E}+09$ \\
\hline
\end{tabular}


Table 7: Comparison of average running time results (seconds) over 30 runs for larger-scale problems with 1000 variables

\begin{tabular}{|c|c|c|c|c|c|c|c|c|c|c|c|c|c|}
\hline ID & Mertic & $\mathrm{HHO}$ & GA & PSO & BBO & FPA & GWO & BAT & FA & CS & MFO & & $\mathrm{DE}$ \\
\hline \multirow[t]{2}{*}{ F1 } & AVG & $2.03 \mathrm{E}+00$ & 3.27E+01 & $8.29 \mathrm{E}+01$ & $1.17 \mathrm{E}+02$ & $2.13 \mathrm{E}+00$ & $4.47 \mathrm{E}+00$ & $1.60 \mathrm{E}+00$ & $5.62 \mathrm{E}+00$ & $5.47 \mathrm{E}+00$ & $3.23 \mathrm{E}+00$ & 2. $21 \mathrm{E}+00$ & 2. $\quad++00$ \\
\hline & STD & $4 \mathrm{E}-01$ & $13 \mathrm{E}+00$ & $04 \mathrm{E}+00$ & $04 \mathrm{E}+00$ & $2.62 \mathrm{E}-01$ & $2.64 \mathrm{E}-01$ & $2.08 \mathrm{E}-01$ & 4.42E-01 & $4.00 \mathrm{E}-01$ & $2.06 \mathrm{E}-01$ & 2 ६2E-01 & $2.70 \mathrm{E}-J 1$ \\
\hline \multirow[t]{2}{*}{$\mathrm{F} 2$} & AVG & $1.70 \mathrm{E}+00$ & $41 \mathrm{E}+01$ & $8.28 \mathrm{E}+01$ & $1.16 \mathrm{E}+02$ & $2.09 \mathrm{E}+00$ & $4.37 \mathrm{E}+00$ & $1.61 \mathrm{E}+00$ & $2.57 \mathrm{E}+00$ & $5.50 \mathrm{E}+00$ & $3.25 \mathrm{E}+0 \mathrm{r}$ & & $2.28 \mathrm{E}+00$ \\
\hline & STD & $37 \mathrm{E}-02$ & $5 \mathrm{E}+00$ & $8 \mathrm{E}+00$ & $6.28 \mathrm{E}+00$ & $8.64 \mathrm{E}-02$ & $1.29 \mathrm{E}-01$ & $1.02 \mathrm{E}-01$ & 3.93E-01 & $3.48 \mathrm{E}-01$ & & 1.19! 01 & $1.16 \mathrm{E}-01$ \\
\hline \multirow[t]{2}{*}{ F3 } & AVG & $7 \mathrm{E}+02$ & $2 \mathrm{E}+02$ & $0 \mathrm{E}+02$ & $1.65 \mathrm{E}+02$ & $5.10 \mathrm{E}+01$ & $5.20 \mathrm{E}+01$ & $5.23 \mathrm{E}+01$ & $3.70 \mathrm{E}+01$ & $1.02 \mathrm{E}+02$ & $5.11 \mathrm{E}$, & $9.7^{r}+01$ & $5.04 \mathrm{E}+01$ \\
\hline & STD & & & & & & & & & & & ? $\quad E+00$ & $1.98 \mathrm{E}+00$ \\
\hline \multirow[t]{2}{*}{$\mathrm{F} 4$} & AVG & & $4 \mathrm{E}+01$ & $24 \mathrm{E}+01$ & $1.18 \mathrm{E}+02$ & $1.90 \mathrm{E}+00$ & & & & & & +00 & $2.21 \mathrm{E}+00$ \\
\hline & STD & & $3 \mathrm{E}+00$ & $91 \mathrm{E}+00$ & $5.48 \mathrm{E}+00$ & $5.83 \mathrm{E}-02$ & $1.36 \mathrm{E}-01$ & $1.02 \mathrm{E}-01$ & & & & $1.05 \mathrm{E}-\mathrm{c}$ & $8.73 \mathrm{E}-02$ \\
\hline \multirow[t]{2}{*}{ F5 } & AVG & & & & & & & & & & $3.31 \mathrm{E}+0 \mathrm{u}$ & $3 \mathrm{E}+00$ & $2.38 \mathrm{E}+00$ \\
\hline & STD & & & & & & & & & & & & $1.30 \mathrm{E}-01$ \\
\hline \multirow[t]{2}{*}{ F6 } & AVG & & & & & & & & & & $3.131 \mathrm{~s}$ & $1.89 \mathrm{E}+00$ & $2.19 \mathrm{E}+00$ \\
\hline & STD & & & & & & & & & & $1.00 \quad-01$ & $3 \mathrm{E}-02$ & $1.02 \mathrm{E}-01$ \\
\hline \multirow[t]{2}{*}{ F7 } & AVG & & $6 \mathrm{E}+01$ & & +02 & -00 & & & & & $5.5 \mathrm{E}+00$ & $7.23 \mathrm{E}+00$ & $4.95 \mathrm{E}+00$ \\
\hline & STD & & & & & & & & & & ${ }^{1} \mathrm{E}-01$ & $1.31 \mathrm{E}-01$ & $1.43 \mathrm{E}-01$ \\
\hline \multirow[t]{2}{*}{ F8 } & AVG & & & & & & & & & & 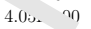 & $3.84 \mathrm{E}+00$ & $\mathrm{E}+00$ \\
\hline & STD & & $8 E+00$ & $0 \mathrm{E}+00$ & $5.52 \mathrm{E}+00$ & & & & & & $120 \mathrm{E}-01$ & & \\
\hline \multirow[t]{2}{*}{ F9 } & AVG & & & & & & & & & & & $2.70 \mathrm{E}+00$ & $\mathrm{E}+00$ \\
\hline & & & & & & & & & & & & & \\
\hline \multirow[t]{2}{*}{ F10 } & AVG & & & & & & & & & & $4 \div \quad \mathrm{E}+00$ & & \\
\hline & STD & & & & & & & & & & $\angle 1 \mathrm{E}-01$ & $5.39 \mathrm{E}-01$ & $3.01 \mathrm{E}-01$ \\
\hline \multirow[t]{2}{*}{ F11 } & AVG & & & & & & & & $6.03 \mathrm{~F}$ u & $6.43 \mathrm{E}+00$ & & $3.03 \mathrm{E}+00$ & \\
\hline & STD & & & & & & & & $2.55,-01$ & & & & $5 \mathrm{E}-02$ \\
\hline \multirow[t]{2}{*}{ F12 } & AVG & & & & & & & & & $1.90 \mathrm{E}-11$ & & $1.53 \mathrm{E}+01$ & $9.14 \mathrm{E}+00$ \\
\hline & STD & & & & & & & & & $\begin{array}{lll}3.53 \mathrm{E} & \text { ग0 }\end{array}$ & & $2.54 \mathrm{E}+00$ & $1.14 \mathrm{E}+00$ \\
\hline \multirow[t]{2}{*}{$\mathrm{F} 13$} & AVG & & & & & & & & $? 4 \mathrm{E}+00$ & $1.8^{9}+01$ & $9.66 \mathrm{E}+00$ & $1.46 \mathrm{E}+01$ & $9.34 \mathrm{E}-$ \\
\hline & STD & & & & & & & & & & $3.91 \mathrm{E}-01$ & $2.24 \mathrm{E}+00$ & \\
\hline
\end{tabular}

always achieved to the best results on F14-F23 probı ns in comparison with other approaches. Based on results for F24-F29 hybrid CM functions in T^h. 3, the HHO is capable of achieving to high-quality solutions and outperforming other compe ${ }^{\cdot{ }^{+}}{ }^{\circ}$ ors. The p-values in Table 24 also confirm the meaningful advantage of HHO compared to vin nntimizers for the majority of cases.

Table 8: Results of beょ \% marı functions (F14-F29)

\begin{tabular}{|c|c|c|c|c|c|c|c|c|c|c|c|c|c|}
\hline \multicolumn{2}{|c|}{ Benchmark } & HHO & GA & PSO & BBO & FPA & GWO & BAT & FA & CS & MFO & TLBO & $\mathrm{DE}$ \\
\hline F14 & $\begin{array}{l}\text { AVG } \\
\text { STD }\end{array}$ & $\begin{array}{l}9.98 \mathrm{E}-01 \\
9.23 \mathrm{E}-01\end{array}$ & $\begin{array}{l}9.98 \mathrm{E}-01 \\
4.52 \mathrm{E}-16\end{array}$ & $\begin{array}{l}1.39 \mathrm{E}+00 \\
4.60 \mathrm{E}-01\end{array}$ & $\begin{array}{l}9.98 \mathrm{E}-01 \\
4.52 \mathrm{E}-16\end{array}$ & $\begin{array}{l}9.98 \mathrm{E}-\mathrm{t}_{-} \\
2.00 \mathrm{E}-04\end{array}$ & 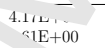 & $\begin{array}{l}1.27 \mathrm{E}+01 \\
6.96 \mathrm{E}+00\end{array}$ & $\begin{array}{l}3.51 \mathrm{E}+00 \\
2.16 \mathrm{E}+00\end{array}$ & $\begin{array}{l}1.27 \mathrm{E}+01 \\
1.81 \mathrm{E}-15\end{array}$ & $\begin{array}{l}2.74 \mathrm{E}+00 \\
1.82 \mathrm{E}+00\end{array}$ & $\begin{array}{l}9.98 \mathrm{E}-01 \\
4.52 \mathrm{E}-16\end{array}$ & $\begin{array}{l}1.23 \mathrm{E}+00 \\
9.23 \mathrm{E}-01\end{array}$ \\
\hline & AVG & $3.10 \mathrm{E}-04$ & $3.33 \mathrm{E}-02$ & $1.61 \mathrm{E}-03$ & $1.66 \mathrm{E}-02$ & $6.88 \mathrm{E}-04$ & $6.24\llcorner\quad 3$ & $3.00 \mathrm{E}-02$ & $1.01 \mathrm{E}-03$ & $3.13 \mathrm{E}-04$ & $2.35 \mathrm{E}-03$ & $1.03 \mathrm{E}-03$ & $5.63 \mathrm{E}-04$ \\
\hline & STD & $1.97 \mathrm{E}-04$ & $2.70 \mathrm{E}-02$ & $4.60 \mathrm{E}-04$ & $8.60 \mathrm{E}-03$ & $1.55 \mathrm{E}-04$ & $1.25 \mathrm{E}-02$ & $3.33 \mathrm{E}-02$ & $4.01 \mathrm{E}-04$ & $2.99 \mathrm{E}-05$ & $4.92 \mathrm{E}-03$ & $3.66 \mathrm{E}-03$ & $2.81 \mathrm{E}-04$ \\
\hline F16 & $\begin{array}{l}\text { AVG } \\
\text { STD }\end{array}$ & $\begin{array}{l}\mathbf{- 1 . 0 3 E + 0 0} \\
6.78 \mathrm{E}-16\end{array}$ & $\begin{array}{l}-3.78 \mathrm{E}-01 \\
3.42 \mathrm{E}-01 \\
\end{array}$ & $\begin{array}{l}-1.03 \mathrm{E}+00 \\
2.95 \mathrm{E}-03\end{array}$ & $\begin{array}{l}-8.30 \mathrm{E}-01 \\
3.16 \mathrm{E}-0^{\prime}\end{array}$ & $\begin{array}{ll}-1.00 \\
6.78 \mathrm{E}-1 \mathrm{c}\end{array}$ & 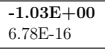 & $\begin{array}{l}-6.87 \mathrm{E}-01 \\
8.18 \mathrm{E}-01 \\
\end{array}$ & 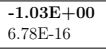 & $\begin{array}{l}\mathbf{- 1 . 0 3 \mathrm { E } + 0 0} \\
6.78 \mathrm{E}-16\end{array}$ & $\begin{array}{l}\mathbf{- 1 . 0 3 E}+\mathbf{0 0} \\
6.78 \mathrm{E}-16\end{array}$ & 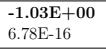 & $\begin{array}{l}\mathbf{- 1 . 0 3 E + 0 0} \\
6.78 \mathrm{E}-16\end{array}$ \\
\hline F17 & $\begin{array}{l}\text { AVG } \\
\text { STD }\end{array}$ & $\begin{array}{l}3.98 \mathrm{E}-01 \\
2.54 \mathrm{E}-06\end{array}$ & $\begin{array}{l}5.24 \mathrm{E}-01 \\
6.06 \mathrm{E}-02\end{array}$ & $\begin{array}{l}4.00 \mathrm{E}-01 \\
1.39 \mathrm{E}-03\end{array}$ & $\begin{array}{l}5.49 \mathrm{E} \\
6.05 \mathrm{E}-\mathrm{u}_{2}\end{array}$ & $\begin{array}{l}3.98 \mathrm{E}-01 \\
1.69 \mathrm{E}-16\end{array}$ & $\begin{array}{l}3.98 \mathrm{E}-01 \\
1.69 \mathrm{E}-16\end{array}$ & $\begin{array}{l}3.98 \mathrm{E}-01 \\
1.58 \mathrm{E}-03\end{array}$ & $\begin{array}{l}\text { 3.98E-01 } \\
1.69 \mathrm{E}-16\end{array}$ & $\begin{array}{l}3.98 \mathrm{E}-01 \\
1.69 \mathrm{E}-16\end{array}$ & $\begin{array}{l}3.98 \mathrm{E}-01 \\
1.69 \mathrm{E}-16\end{array}$ & $\begin{array}{l}3.98 \mathrm{E}-01 \\
1.69 \mathrm{E}-16\end{array}$ & $\begin{array}{l}3.98 \mathrm{E}-01 \\
1.69 \mathrm{E}-16\end{array}$ \\
\hline F18 & $\begin{array}{l}\text { AVG } \\
\text { STD }\end{array}$ & 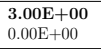 & $\begin{array}{l}3.00 \mathrm{E}+00 \\
0.00 \mathrm{E}+00\end{array}$ & $\begin{array}{l}3.10 \mathrm{E}+00 \\
7.60 \mathrm{E}-02\end{array}$ & $\begin{array}{ll}\text { 3. } & \mathrm{E}+\mathbf{0 0} \\
& 0 \mathrm{E}+00\end{array}$ & $\begin{array}{l}\mathbf{T}_{+}, \overline{\mathrm{T}} \\
0.00 \mathrm{e}\end{array}$ & $\begin{array}{l}3.00 \mathrm{E}+00 \\
4.07 \mathrm{E}-05\end{array}$ & $\begin{array}{l}1.47 \mathrm{E}+01 \\
2.21 \mathrm{E}+01 \\
\end{array}$ & $\begin{array}{l}\mathbf{3 . 0 0 \mathrm { E } + 0 0} \\
0.00 \mathrm{E}+00\end{array}$ & $\begin{array}{l}.00 \mathrm{E}+00 \\
0.00 \mathrm{E}+00\end{array}$ & 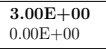 & $\begin{array}{l}\mathbf{3 . 0 0 \mathrm { E } + 0 0} \\
0.00 \mathrm{E}+00\end{array}$ & $\begin{array}{l}\mathbf{3 . 0 0 E}+\mathbf{0 0} \\
0.00 \mathrm{E}+00\end{array}$ \\
\hline F19 & $\begin{array}{l}\text { AVG } \\
\text { STD }\end{array}$ & $\begin{array}{l}-3.86 \mathrm{E}+00 \\
2.44 \mathrm{E}-03\end{array}$ & $\begin{array}{l}-3.42 \mathrm{E}+00 \\
3.03 \mathrm{E}-01 \\
\end{array}$ & 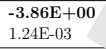 & $\begin{array}{l}3 \overline{78 \mathrm{E}+} \\
1.26 \mathrm{E}-\end{array}$ & $\begin{array}{l}-3.86 \mathrm{E}+00 \\
3.1-15\end{array}$ & $\begin{array}{l}-3.86 \mathrm{E}+00 \\
3.14 \mathrm{E}-03\end{array}$ & $\begin{array}{l}-3.84 \mathrm{E}+00 \\
1.41 \mathrm{E}-01 \\
\end{array}$ & $\begin{array}{l}-3.86 \mathrm{E}+00 \\
3.16 \mathrm{E}-15\end{array}$ & $\begin{array}{l}\mathbf{- 3 . 8 6 \mathrm { E } + 0 0} \\
3.16 \mathrm{E}-15 \\
\end{array}$ & $\begin{array}{l}-3.86 \mathrm{E}+00 \\
1.44 \mathrm{E}-03\end{array}$ & $\begin{array}{l}-3.86 \mathrm{E}+00 \\
3.16 \mathrm{E}-15\end{array}$ & $\begin{array}{l}-3.86 \mathrm{E}+00 \\
3.16 \mathrm{E}-15\end{array}$ \\
\hline F20 & $\begin{array}{l}\text { AVG } \\
\text { STD }\end{array}$ & $\begin{array}{l}-3.322 \\
0.137406 \\
\end{array}$ & $\begin{array}{l}-1.61351 \\
0.46049 \\
\end{array}$ & $\begin{array}{c}-3.11088 \\
0.02912 \\
\end{array}$ & $\begin{array}{l}-2 . \\
0.3578\end{array}$ & $\begin{array}{c}.2951 \\
.019514 \\
\end{array}$ & $\begin{array}{l}-3.25866 \\
0.064305 \\
\end{array}$ & $\begin{array}{l}-3.2546 \\
0.058943 \\
\end{array}$ & $\begin{array}{c}-3.28105 \\
0.063635 \\
\end{array}$ & $\begin{array}{l}-3.322 \\
1.77636 \mathrm{E}-15\end{array}$ & $\begin{array}{c}-3.23509 \\
0.064223 \\
\end{array}$ & $\begin{array}{l}-3.24362 \\
0.15125 \\
\end{array}$ & $\begin{array}{c}-3.27048 \\
0.058919 \\
\end{array}$ \\
\hline F21 & $\begin{array}{l}\text { AVG } \\
\text { STD }\end{array}$ & $\begin{array}{l}-\mathbf{- 1 0 . 1 4 5 1} \\
0.885673\end{array}$ & $\begin{array}{l}-6.66177 \\
3.732521\end{array}$ & $\begin{array}{ll}-4.14^{-1} \\
0.9^{7}, 78\end{array}$ & $\begin{array}{l}-8.31508 \\
2.883867\end{array}$ & $\begin{array}{l}-5.21514 \\
0.008154\end{array}$ & $\begin{array}{l}-8.64121 \\
2.563356\end{array}$ & $\begin{array}{l}-4.2661 \\
2.554009\end{array}$ & $\begin{array}{l}-7.67362 \\
3.50697\end{array}$ & $\begin{array}{l}-5.0552 \\
1.77636 \mathrm{E}-15\end{array}$ & $\begin{array}{l}-6.8859 \\
3.18186\end{array}$ & $\begin{array}{l}-8.64525 \\
1.76521\end{array}$ & $\begin{array}{l}-9.64796 \\
1.51572\end{array}$ \\
\hline $\mathrm{F} 22$ & $\begin{array}{l}\text { AVG } \\
\text { STD }\end{array}$ & $\begin{array}{l}-10.4015 \\
1.352375\end{array}$ & $\begin{array}{l}-5.58399 \\
2.605837\end{array}$ & 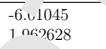 & $\begin{array}{l}-2.798 \\
2.597<\checkmark 8\end{array}$ & $\begin{array}{l}-5.34373 \\
0.053685\end{array}$ & $\begin{array}{l}-10.4014 \\
0.000678\end{array}$ & $\begin{array}{l}-5.60638 \\
3.022612\end{array}$ & $\begin{array}{l}-9.63827 \\
2.293901\end{array}$ & $\begin{array}{l}5.0877 \\
8.88178 \mathrm{E}-16\end{array}$ & $\begin{array}{l}-8.26492 \\
3.076809\end{array}$ & $\begin{array}{l}-10.2251 \\
0.007265\end{array}$ & $\begin{array}{c}-9.74807 \\
1.987703\end{array}$ \\
\hline F23 & $\begin{array}{l}\text { AVG } \\
\text { STD }\end{array}$ & $\begin{array}{l}\mathbf{- 1 0 . 5 3 6 4} \\
0.927655 \\
\end{array}$ & $\begin{array}{l}-4.69882 \\
3.256702 \\
\end{array}$ & $\begin{array}{l}-4.72, \\
1.7426\end{array}$ & $\begin{array}{l}-6.2351 \\
3.78462 \\
\end{array}$ & $\begin{array}{l}-5.29437 \\
0.356377\end{array}$ & $\begin{array}{l}-10.0836 \\
1.721889\end{array}$ & $\begin{array}{l}-3.97284 \\
3.008279 \\
\end{array}$ & $\begin{array}{l}-9.75489 \\
2.345487\end{array}$ & $\begin{array}{l}-5.1285 \\
1.77636 \mathrm{E}-15\end{array}$ & $\begin{array}{l}-7.65923 \\
3.576927 \\
\end{array}$ & $\begin{array}{l}-10.0752 \\
1.696222 \\
\end{array}$ & $\begin{array}{l}\mathbf{- 1 0 . 5 3 6 4} \\
8.88 \mathrm{E}-15\end{array}$ \\
\hline F24 & $\begin{array}{l}\text { AVG } \\
\text { STD }\end{array}$ & $\begin{array}{l}\mathbf{3 9 6 . 8 2 5 6} \\
79.58214 \\
\end{array}$ & $\begin{array}{l}626.838 \\
101.2255\end{array}$ & $\begin{array}{ll}768, & 75 \\
& 1641\end{array}$ & $\begin{array}{l}493.0129 \\
102.6058 \\
\end{array}$ & $\begin{array}{l}518.7886 \\
47.84199 \\
\end{array}$ & $\begin{array}{l}486.5743 \\
142.9028 \\
\end{array}$ & $\begin{array}{l}1291.474 \\
150.4189 \\
\end{array}$ & $\begin{array}{l}471.9752 \\
252.1018 \\
\end{array}$ & $\begin{array}{l}469.0141 \\
60.62538\end{array}$ & $\begin{array}{l}412.4627 \\
68.38819 \\
\end{array}$ & $\begin{array}{l}612.5569 \\
123.2403 \\
\end{array}$ & $\begin{array}{l}431.0767 \\
64.1864\end{array}$ \\
\hline F25 & $\begin{array}{l}\text { AVG } \\
\text { STD }\end{array}$ & $\begin{array}{l}910 \\
0\end{array}$ & $\begin{array}{lr}999 & , 98 \\
25 & 4366\end{array}$ & $\begin{array}{l}1184.4 \\
33.02676\end{array}$ & $\begin{array}{l}935.4693 \\
9.61349\end{array}$ & $\begin{array}{l}1023.799 \\
31.85965\end{array}$ & $\begin{array}{l}985.4172 \\
29.95368\end{array}$ & $\begin{array}{l}1463.423 \\
68.41612\end{array}$ & $\begin{array}{l}553.8902 \\
11.74911\end{array}$ & $\begin{array}{l}910.1008 \\
0.036659\end{array}$ & $\begin{array}{l}947.9322 \\
27.06628\end{array}$ & $\begin{array}{l}967.088 \\
27.39906\end{array}$ & $\begin{array}{l}917.6204 \\
1.052473\end{array}$ \\
\hline F26 & $\begin{array}{l}\text { AVG } \\
\text { STD }\end{array}$ & $\begin{array}{l}910 \\
0 \\
\end{array}$ & $\begin{array}{r}8.9091 \\
-\quad 781^{\circ} \\
\end{array}$ & $\begin{array}{l}1178 . .^{7} \\
35.2^{\prime}\end{array}$ & $\begin{array}{l}934.2718 \\
8.253209\end{array}$ & $\begin{array}{l}1018.002 \\
34.87908\end{array}$ & $\begin{array}{l}973.5362 \\
22.45008\end{array}$ & $\begin{array}{l}1480.683 \\
45.55006\end{array}$ & $\begin{array}{l}953.5493 \\
14.086\end{array}$ & $\begin{array}{l}910.1252 \\
0.047205\end{array}$ & $\begin{array}{l}940.1221 \\
21.68256\end{array}$ & $\begin{array}{l}983.774 \\
45.32275\end{array}$ & $\begin{array}{l}917.346 \\
0.897882 \\
\end{array}$ \\
\hline $\mathrm{F} 27$ & $\begin{array}{l}\text { AVG } \\
\text { STD }\end{array}$ & $\begin{array}{l}910 \\
0\end{array}$ & $\begin{array}{c}1002.1 \% \\
6.66321\end{array}$ & $\begin{array}{r}11 \\
\quad .088 \\
97978\end{array}$ & $\begin{array}{l}939.7644 \\
23.07814\end{array}$ & $\begin{array}{l}1010.392 \\
31.51188\end{array}$ & $\begin{array}{l}969.8538 \\
19.51721\end{array}$ & $\begin{array}{l}1477.919 \\
60.58827\end{array}$ & $\begin{array}{l}947.7667 \\
11.18408\end{array}$ & $\begin{array}{l}910.1233 \\
0.049732\end{array}$ & $\begin{array}{l}945.4266 \\
26.79031\end{array}$ & $\begin{array}{l}978.7344 \\
38.22729\end{array}$ & $\begin{array}{l}917.3067 \\
0.861945\end{array}$ \\
\hline F28 & $\begin{array}{l}\text { AVG } \\
\text { STD }\end{array}$ & $\begin{array}{ll}860.8^{\prime} & 5 \\
0.651: & 2\end{array}$ & $\begin{array}{l}1512.4 \\
94.6455 \mathrm{~s}\end{array}$ & $\begin{array}{l}1711.981 \\
35.18377\end{array}$ & $\begin{array}{l}1068.631 \\
201.9045\end{array}$ & $\begin{array}{l}1539.357 \\
42.93441\end{array}$ & $\begin{array}{l}1337.671 \\
191.0662\end{array}$ & $\begin{array}{l}1961.526 \\
58.46188\end{array}$ & $\begin{array}{l}1016.389 \\
270.6854\end{array}$ & $\begin{array}{l}1340.078 \\
134.183\end{array}$ & $\begin{array}{l}1455.918 \\
36.06884\end{array}$ & $\begin{array}{l}1471.879 \\
268.6238\end{array}$ & $\begin{array}{l}1553.993 \\
96.35255\end{array}$ \\
\hline F29 & $\begin{array}{l}\text { AVG } \\
\text { STD }\end{array}$ & $\begin{array}{l}558.9 \mathrm{e} \\
5.112352\end{array}$ & $\begin{array}{l}1937.396 \\
11.259^{\circ}\end{array}$ & $\begin{array}{l}2101.145 \\
29.74533\end{array}$ & $\begin{array}{l}1897.439 \\
8.823239\end{array}$ & $\begin{array}{l}2033.614 \\
30.2875\end{array}$ & $\begin{array}{l}1909.091 \\
6.567542\end{array}$ & $\begin{array}{l}2221.404 \\
35.54849\end{array}$ & $\begin{array}{l}1986.206 \\
18.88722\end{array}$ & $\begin{array}{l}1903.852 \\
185.7944\end{array}$ & $\begin{array}{l}1882.974 \\
6.528261\end{array}$ & $\begin{array}{l}1883.773 \\
3.493192\end{array}$ & $\begin{array}{l}1897.031 \\
4.203909\end{array}$ \\
\hline
\end{tabular}

\subsection{Engineeriı。' 'enchmark sets}

In this sec 'ic 1 , the proposed HHO is applied to six well-known benchmark engineering problems. Tackling , ngineering design tasks using P-metaheuristics is a well-regarded research direction in the previous works $[60,61]$. The results of HHO is compared to various conventional and modified optimizers proposed in previous studies. Table 9 tabulates the details of the tackled engineering design tasks. 
Table 9: Brief description of the tackled engineering design tasks. (D: dimension, CV: continuous variables, DV:Discrete variables, NC: Number of constraints, AC: Active constraints, F/S: ratio of the feasible solutions in the solution domain (F) to the whole search domain(S), OB: Objective.)

\begin{tabular}{|c|c|c|c|c|c|c|c|c|}
\hline No. & Name & $\mathrm{D}$ & $\mathrm{CV}$ & DV & $\mathrm{NC}$ & $\mathrm{AC}$ & $\mathrm{F} / \mathrm{S}$ & OB \\
\hline 1 & Three-bar truss & 2 & 2 & 0 & 3 & NA & NA & Minimizr we \\
\hline 2 & Tension/compression spring & 3 & 3 & 0 & 4 & 2 & 0.01 & Minimi e we: ht \\
\hline 3 & Pressure vessel & 4 & 2 & 2 & 4 & 2 & 0.40 & Minimize c t \\
\hline 4 & Welded beam & 4 & 4 & 0 & 7 & 2 & 0.035 & Minimı $_{\llcorner}$cost \\
\hline 5 & Multi-plate disc clutch brake & 5 & 0 & 5 & 8 & 1 & 0.700 & $\mathrm{M}: \ldots$ ize wt. jht \\
\hline 6 & Rolling element bearing & 10 & 9 & 1 & 9 & 4 & 0.015 & taxir su drnamic load \\
\hline
\end{tabular}

\subsubsection{Three-bar truss design problem}

This problem can be regarded as one of the most studied ce res ir previous works [62]. This problem can be described mathematically as follows:

$$
\begin{aligned}
& \text { Consider } \quad \vec{X}=\left[x_{1} x_{2}\right]=\left[A_{1} A_{2}\right] \text {, } \\
& \text { Minimise } f(\vec{X})=\left(2 \sqrt{2} X_{1}+v_{\curvearrowright}\right) \wedge 1 \text {, } \\
& \text { Subject to } \quad g_{1}(\vec{X})=\frac{\sqrt{2} x_{1} x_{2}}{\sqrt{2} x^{2}+\gamma_{r_{1}} x_{2}} P-\sigma \leq 0 \text {, }
\end{aligned}
$$

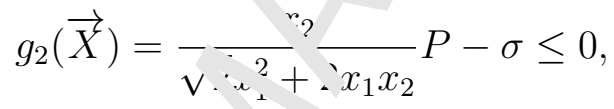

$$
\begin{aligned}
& g_{3}(\vec{X})=\frac{1}{\sqrt{2} r_{2}+x_{1}} P-\sigma \leq 0,
\end{aligned}
$$

Variable range $\quad 0 \leq x, x_{2} \leq 1$,

where $\quad 1=100 \mathrm{cr}, \quad P=2 \mathrm{KN} / \mathrm{cm}^{2}, \sigma=2 \mathrm{KN} / \mathrm{cm}^{2}$

Figure 13 demonstrates the sh pe f the formulated truss and the related forces on this structure. With regard to Fig. 13 and $\omega_{\text {- }}$ ' corr ulation, we have two parameters: the area of bars 1 and 3 and area of bar 2 . The objec ${ }^{+}$ve of thıs task is to minimize the total weight of the structure. In addition, this design case has seveı, ' constraints including stress, deflection, and buckling.

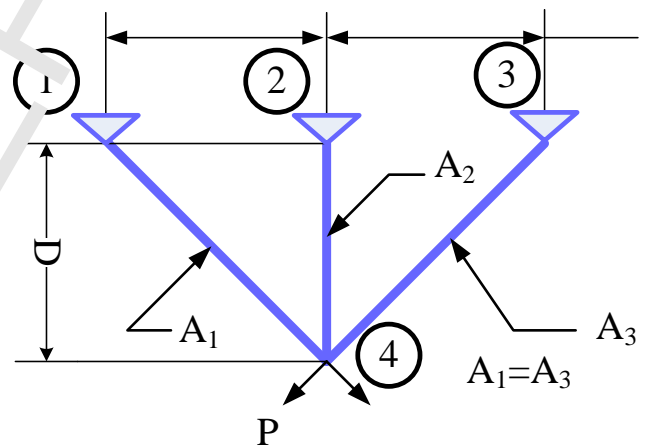

Figure 13: Three-bar truss design problem

The HHO is applied to this case based on 30 independent runs with 30 hawks and 500 iterations in each run. Since this benchmark case has some constraints, we need to integrate the HHO with 
a constraint handling technique. For the sake of simplicity, we used a barrier penalty approach [63] in the HHO. The results of HHO are compared to those reported for DF DS [64], MVO [65],

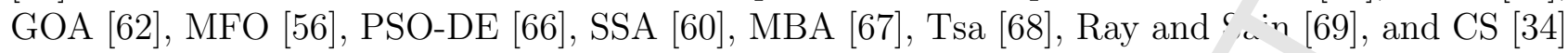
in previous literature. Table 10 shows the detailed results of the proposed HHO con-pared to other techniques. Based on the results in Table 10, it is observed that HHO car rer zal very competitive results compared to DEDS, PSO-DE, and SSA algorithms. Additionally, t ae HHO outperforms other optimizers significantly. The results obtained show that the HHO 1 r rapable of dealing with a constrained space.

Table 10: Comparison of results for three-bar truss desig. rroblem.

\begin{tabular}{|c|c|c|c|}
\hline \multirow[t]{2}{*}{ Algorithm } & \multicolumn{2}{|c|}{ Optimal values for variables } & \multirow[t]{2}{*}{$\overline{\mathrm{Op}} \overline{\text { mal weight }}$} \\
\hline & $x_{1}$ & $x_{2}$ & \\
\hline $\mathrm{HHO}$ & 0.788662816 & $0.40828313383^{n \curvearrowleft} 7$ & - - $3 . \overline{8958434}$ \\
\hline DEDS [64] & 0.78867513 & 0.40824828 & 263.8958434 \\
\hline MVO $[65]$ & 0.78860276 & $0.4084530700 \iota^{\urcorner n}$, & 263.8958499 \\
\hline GOA [62] & 0.788897555578973 & $0.4076195 i \cdot 1515 ?$ & 263.895881496069 \\
\hline $\mathrm{MFO}[56]$ & 0.788244771 & $0.409466905784, ' 1$ & 263.8959797 \\
\hline PSO-DE [66] & 0.7886751 & $0.40824 ?$ & 263.8958433 \\
\hline SSA $[60]$ & 0.788665414 & 0.408 .75784 .115 .7 & 263.8958434 \\
\hline $\mathrm{MBA}[67]$ & 0.7885650 & 0.408559 , & 263.8958522 \\
\hline Tsa [68] & 0.788 & $0 \cdots$ & 263.68 \\
\hline Ray and Sain [69] & 0.795 & 0.395 & 264.3 \\
\hline CS $[34]$ & 0.78867 & ก 1n902 & 263.9716 \\
\hline
\end{tabular}

\subsubsection{Tension/compression spring desig ?}

In this case, our intention is to minim ino th weight of a spring. Design variables for this case are wire diameter $(d)$, mean coil diamete. $(D)$, and the number of active coils $(N)$. For this case, the constraints on shear stress, suroe frequency, and minimum deflection should be satisfied during the weight optimization. The $r$ oject ${ }^{2} \mathrm{e}$ and constraints of this problem can be formulated as follows:

$$
\begin{aligned}
& \text { Cor sider } \vec{\sim}=\left[z_{1} z_{2} z_{3}\right]=[d D N], \\
& \text { Ninin. e } f(\vec{z})=\left(z_{3}+2\right) z_{2} z_{1}^{2}, \\
& \text { su. ject to } \\
& g_{1}(\vec{z})=1-\frac{z_{2}^{3} z_{3}}{71785 z_{1}^{4}} \leq 0, \\
& \neg(\vec{z})=\frac{4 z_{2}^{2}-z_{1} z_{2}}{12566\left(z_{2} z_{1}^{3}-z_{1}^{4}\right)}+\frac{1}{5108 z_{1}^{2}} \leq 0, \\
& g_{3}(\vec{z})=1-\frac{140.45 z_{1}}{z_{2}^{2} z_{3}} \leq 0 \\
& g_{4}(\vec{z})=\frac{z_{1}+z_{2}}{1.5}-1 \leq 0,
\end{aligned}
$$

There are eral optimizers previously applied to this case such as the SSA [60], TEO [70], MFO [56], SFS [ 1], GWO [55], WOA [18], method presented by Arora [72], GA2 [73], GA3 [74], method presented by Belegundu [75], CPSO [76], DEDS [64], GSA [25], DELC [77], HEAA [78], WEO [79], BA [80], ESs [81], Rank-iMDDE [82], CWCA [14], and WCA [61]. The results of HHO are compared to the aforementioned techniques in Table 11. 
Table 11: Comparison of results for tension/compression spring problem.

\begin{tabular}{|c|c|c|c|c|}
\hline Algorithms & $d$ & $D$ & $N$ & Optims $\overline{\operatorname{cost}}$ \\
\hline $\mathrm{HHO}$ & 0.051796393 & 0.359305355 & 11.138859 & $0.012^{r}$ ๘13 \\
\hline $\mathrm{SSA}[60]$ & 0.051207 & 0.345215 & 12.004032 & $0.01 \angle 0763$ \\
\hline TEO [70] & 0.051775 & 0.3587919 & 11.16839 & $0 . \Gamma-\angle 5$ \\
\hline $\mathrm{MFO}[56]$ & 0.051994457 & 0.36410932 & 10.868422 & ○ J126 39 \\
\hline SFS [71] & 0.051689061 & 0.356717736 & 11.288966 & $0.4 . ?, 65233$ \\
\hline GWO [55] & 0.05169 & 0.356737 & 11.28885 & n126u \\
\hline WOA [18] & 0.051207 & 0.345215 & 12.004032 & $0.0_{\perp} .763$ \\
\hline Arora [72] & 0.053396 & 0.399180 & 9.185400 & ^12730 \\
\hline GA2 [73] & 0.051480 & 0.351661 & $11.6322 \mathrm{C}$ & 0.012704 \\
\hline GA3 [74] & 0.051989 & 0.363965 & 10.890592 & ก.012681 \\
\hline Belegundu [75] & 0.05 & 0.315900 & 14.25000 & 0.012833 \\
\hline CPSO $[76]$ & 0.051728 & 0.357644 & 11.24543 & 0.012674 \\
\hline DEDS [64] & 0.051689 & 0.356717 & 11.28 ר 75.5 & 0.012665 \\
\hline GSA $[25]$ & 0.050276 & 0.323680 & .3 .525410 & 0.012702 \\
\hline DELC [77] & 0.051689 & 0.356717 & 11. 896 & 0.012665 \\
\hline HEAA [78] & 0.051689 & 0.356729 & $11.2882^{r} 3$ & 0.012665 \\
\hline WEO [79] & 0.051685 & 0.356630 & 11.294103 & 0.012665 \\
\hline $\mathrm{BA}[80]$ & 0.05169 & 0.35673 & 11.. 985 & 0.012665 \\
\hline $\operatorname{ESs}[81]$ & 0.051643 & 0.355360 & 1197926 & 0.012698 \\
\hline Rank-iMDDE [82] & 0.051689 & 0.3567171 ১ & $\perp 1.288999$ & 0.012665 \\
\hline CWCA [14] & 0.051709 & 0.35710734 & 11.270826 & 0.012672 \\
\hline WCA [61] & 0.05168 & $0.3565 \_$ & 11.30041 & 0.012665 \\
\hline
\end{tabular}

Table 11 shows that the proposed HHO can $a^{h}$ eve to high quality solutions very effectively when tackling this benchmark problem and in nos s the best design. It is evident that results of HHO are very competitive to those of SFS an, ${ }^{1} \mathrm{IEO}$.

\subsubsection{Pressure vessel design problem}

In this well-regarded case, we min inlz the fabrication cost and it has four parameters and constraints. The variables of this c. se are $\left(x_{1}-x_{4}\right): T_{s}\left(x_{1}\right.$, thickness of the shell), $T_{h}\left(x_{2}\right.$, thickness of the head), $r\left(x_{3}\right.$, inner radius, $L\left(x_{4}\right.$, length of the section without the head). The overall configuration of this prob $^{\top} \mathrm{m} / \mathrm{s}$ sh Jwn in Fig. 14. The formulation of this test case is as
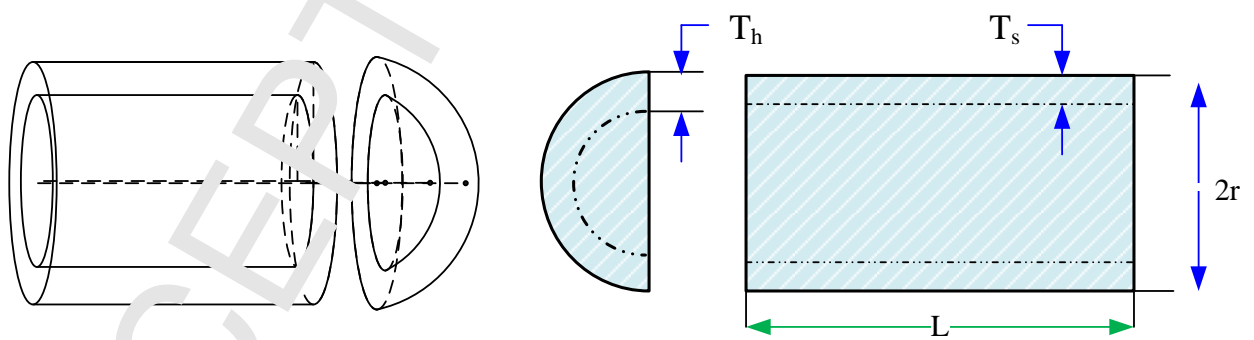

Figure 14: Pressure vessel problem 
follows:

$$
\begin{aligned}
& \text { Consider } \vec{z}=\left[z_{1} z_{2} z_{3} z_{4}\right]=\left[T_{s} T_{h} R L\right] \\
& \text { Minimize } f(\vec{z})=0.6224 z_{1} z_{3} z_{4}+1.7781 z_{2} z_{2}^{3}+3.1661 z_{1}^{2} z_{4}+19.84 z_{1}^{2} z_{3} \\
& \text { Subject to } \\
& g_{1}(\vec{z})=-z_{1}+0.0193 z_{3} \leq 0 \\
& g_{2}(\vec{z})=-z_{3}+0.00954 z_{3} \leq 0 \\
& g_{3}(\vec{z})=-\Pi z_{3}^{2} z_{4}-\frac{4}{3} \Pi z_{3}^{3}+1,296,000 \leq 0 \\
& g_{4}(\vec{z})=z_{4}-240 \leq 0
\end{aligned}
$$

The design space for this case is limited to: $0 \leq z_{1}, z_{2} \leq y 9,0 \leq z_{3}, z_{4} \leq 200$. The results of HHO are compared to those of GWO [55], GA [73], HPSO [8 , G QPSO [84], WEO [79], IACO

\begin{tabular}{|c|c|c|c|c|c|}
\hline Algorithms & $T_{s}\left(x_{1}\right)$ & 7 & $R\left(x_{3}\right)$ & $L\left(x_{4}\right)$ & Optimal cost \\
\hline $\mathrm{HHO}$ & 0.81758383 & 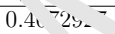 & 42.09174576 & 176.7196352 & 6000.46259 \\
\hline GWO [55] & 0.8125 & 0.434 & 42.089181 & 176.758731 & 6051.5639 \\
\hline GA $[73]$ & 0.812500 & . & 42.097398 & 176.654050 & 6059.9463 \\
\hline HPSO [83] & 0.812500 & ${ }^{137500}$ & 42.0984 & 176.6366 & 6059.7143 \\
\hline G-QPSO [84] & 0.812500 & $0.43 ، 0$ & 42.0984 & 176.6372 & 6059.7208 \\
\hline WEO [79] & 0.812500 & 0.437500 & 42.098444 & 176.636622 & 6059.71 \\
\hline IACO [85] & 0.812 u & 0.437500 & 42.098353 & 176.637751 & 6059.7258 \\
\hline $\mathrm{BA}[80]$ & 0.81 .500 & .437500 & 42.098445 & 176.636595 & 6059.7143 \\
\hline MFO [56] & 0.81 & .4375 & 42.098445 & 176.636596 & 6059.7143 \\
\hline CSS [86] & r 812500 & 0.437500 & 42.103624 & 176.572656 & 6059.0888 \\
\hline ESs [81] & J.812 30 & 0.437500 & 42.098087 & 176.640518 & 6059.7456 \\
\hline CPSO $[76]$ & $0.81 j 00$ & 0.437500 & 42.091266 & 176.746500 & 6061.0777 \\
\hline BIANCA [87] & $\checkmark 2500$ & 0.437500 & 42.096800 & 176.658000 & 6059.9384 \\
\hline MDDE [88] & 0.81 . ר & 0.437500 & 42.098446 & 176.636047 & 6059.701660 \\
\hline DELC [77] & 0.812500 & 0.437500 & 42.0984456 & 176.6365958 & 6059.7143 \\
\hline WOA [18] & 812500 & 0.437500 & 42.0982699 & 176.638998 & 6059.7410 \\
\hline GA3 [74] & 0.8 .2500 & 0.437500 & 42.0974 & 176.6540 & 6059.9463 \\
\hline Lagrangian multiplier (Ke nan $\left.{ }^{\circ} 18\right]$ & 1.125000 & 0.625000 & 58.291000 & 43.6900000 & 7198.0428 \\
\hline Branch-bound (Sandgrer [18] & 1.125000 & 0.625000 & 47.700000 & 117.701000 & 8129.1036 \\
\hline
\end{tabular}

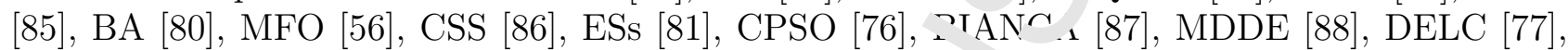
WOA [18], GA3 [74], Lagrangian multiplier (Kannan) [18], a d Branch-bound (Sandgren) [18]. Table 12 reports the optimum designs attained by HHC an $r$ listed optimizers. Inspecting the results in Table 12, we detected that the HHO is the bes optimizer in dealing with problems and can attain superior results compared to other tech.. rues.

Table 12: Comparison of results $\wedge^{` r} \mu_{1} \ldots$ re vessel design problem

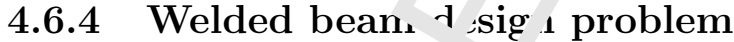

Purpose of the ${ }^{-11}-k_{n} w_{n} \Omega$ engineering case is to discover the best manufacturing cost with regard to a series of design constraints. A schematic view of this problem is illustrated in Fig. 15. The design variahles an tiickness of weld $(h)$, length $(l)$, height $(t)$, and thickness of the bar $(b)$.

This case ca 1 be $f_{l}$ "mulated as follows: 


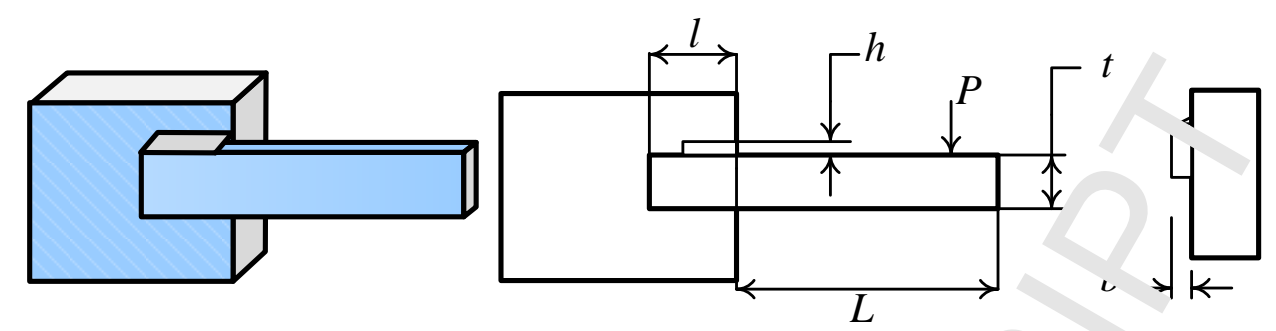

Figure 15: Welded beam design problem

Consider $\vec{z}=\left[z_{1}, z_{2}, z_{3}, z_{4}\right]=[h, l, t, b]$,

Minimize $f(\vec{z})=1.10471 z_{1}^{2} z_{2}+0.04811 z_{3} z_{4}\left(14.6, z_{2}\right)$

Subject to

$g_{1}(\vec{z})=\tau(\vec{z})-\tau_{\max } \leq 0$,

$g_{2}(\vec{z})=\sigma(\vec{z})-\sigma_{\max } \leq 0$,

$g_{3}(\vec{z})=\delta(\vec{z})-\delta_{\max } \leq 0$,

$g_{4}(\vec{z})=z_{1}-z_{4} \leq 0$,

$g_{5}(\vec{z})=P-P_{c}(\vec{z}) \leq 0$,

$g_{6}(\vec{z})=0.125-z_{1} \leq 0$,

$\left.g_{7}(\vec{z})=1.10471 z_{1}^{2}+0.04811 z_{3} \ldots \ldots \quad-z_{2}\right)-5.0 \leq 0$,

Variable range

$$
0.05 \leq z_{1} \leq 2.00, \quad r .25-z_{2} \leq 1.30, \quad 2.00 \leq z_{3} \leq 15.0,
$$

where

$$
\begin{aligned}
& \tau(\vec{z})=\sqrt{\tau^{\prime 2}+2 \tau^{\prime} \tau^{\prime \prime}} \frac{2}{2} \cdot \tau^{\prime \prime \prime}, \tau^{\prime}=\frac{P}{\sqrt{2} z_{1} z_{2}}, \tau^{\prime \prime}=\frac{M R}{J}, M=P\left(L+\frac{z_{2}}{2}\right), \\
& R=\sqrt{\frac{z_{2}^{2}}{4}+\left(\frac{z_{1}+3}{n}\right)^{2},} \cdot \dot{\omega}=2\left\{\sqrt{2} z_{1} z_{2}\left[\frac{z_{2}^{2}}{12}+\left(\frac{z_{1}+z_{3}}{2}\right)^{2}\right]\right\}, \sigma(\vec{z})=\frac{6 P L}{z_{4} z_{3}^{2}}, \\
& \delta(\vec{z})=\frac{4 P L^{3}}{E z^{3} z_{4}}, P_{c}(\stackrel{\vec{\sim}}{)})=\frac{4.013 E \sqrt{\frac{z_{3}^{2} z_{4}^{6}}{36}}}{L^{2}}\left(1-\frac{z_{3}}{2 L} \sqrt{\frac{E}{4 G}}\right) \text {, } \\
& P=6000 / h L-1 \text { in }, E=30 \times 10^{6} \text { psi, G }=12 \times 10^{6} \text { psi, }
\end{aligned}
$$

The optimal , esults of HHO versus those attained by RANDOM [89], DAVID [89], SIMPLEX [89], APPROX 99], G 1 [73], GA2 [63], HS [90], GSA [18], ESs [81], and CDE [91] are represented in Table 13. Frol ${ }^{2}$ able 13, it can be seen that the proposed HHO can reveal the best design settings with the $11+n$ nimum fitness value compared to other optimizers.

\subsubsection{Multi-pıate disc clutch brake}

In this discrete benchmark task, the intention is to optimize the total weight of a multiple disc clutch brake with regard to five variables: actuating force, inner and outer radius, number of 
Table 13: Comparison of results for welded beam design problem

\begin{tabular}{|c|c|c|c|c|c|}
\hline Algorithm & $h$ & $l$ & $t$ & $b$ & Opt nal cost \\
\hline $\mathrm{HHO}$ & 0.204039 & 3.531061 & 9.027463 & 0.206147 & 1.7 \\
\hline RANDOM [89] & 0.4575 & 4.7313 & 5.0853 & 0.66 & 4.1185 \\
\hline DAVID [89] & 0.2434 & 6.2552 & 8.2915 & 0.2444 & L.3. 11 \\
\hline SIMPLEX [89] & 0.2792 & 5.6256 & 7.7512 & 0.2796 & $2.5,07$ \\
\hline APPROX [89] & 0.2444 & 6.2189 & 8.2915 & 0.2444 & ¿. Q. 15 \\
\hline GA1 [73] & 0.248900 & 6.173000 & 8.178900 & 0.253300 & 433116 \\
\hline GA2 [63] & 0.208800 & 3.420500 & 8.997500 & $0.21000 \mathrm{r}$ & 1.748310 \\
\hline HS [90] & 0.2442 & 6.2231 & 8.2915 & 0.2443 & L.j807 \\
\hline GSA [18] & 0.182129 & 3.856979 & 10 & $0.20237 b$ & 1.879952 \\
\hline ESs [81] & 0.199742 & 3.61206 & 9.0375 & $0.2^{r}$ ง 32 & 1.7373 \\
\hline CDE [91] & 0.203137 & 3.542998 & 9.033498 & $0 . \therefore 06179$ & 1.733462 \\
\hline
\end{tabular}

friction surfaces, and thickness of discs [92].

This problem has eight constraints according to the c. nditinn, of geometry and operating requirements. The feasible area for this case includes practically $.7 \%$ of the solution space. However, there are few works that considered this problem in thes test. The optimal results of proposed HHO in compared to those revealed by TLBO [93], Wㄴ [61], and PVS [92] algorithms. Table 14 shows the attained results of different optimiz, iv unis test case. From Table 14, we can recognize that the HHO attains the best rank and can - itperform the well-known TLBO, WCA, and PVS in terms of quality of solutions.

$f(x)=\Pi\left(r_{o}^{2}-r_{i}^{2}\right) t(Z+1) \rho$

subject to:

$g_{1}(x)=r_{o}-r_{i}-\Delta r \geq 0$

$g_{2}(x)=l_{\max }-(Z+1)(t+\delta) \geq 1$

$g_{3}(x)=P_{\max }-P_{r z} \geq 0$

$g_{4}(x)=P_{\max } v_{s r \max }-P_{r z} \nu_{s r}=r$

$g_{5}(x)=v_{s r \max }-v_{s r} \geq 0$

$g_{6}=T_{\max }-T \geq 0$

$g_{7}(x)=M_{h}-s M_{s} \geq{ }^{\prime}$

$g_{8}(x)=T \geq 0$

where,

$M_{h}=\frac{2}{3} \mu F Z \frac{r_{o}^{3}-r_{i}^{2}}{r} \frac{r_{i}^{3}}{-r_{r z}}=\frac{F}{\Pi\left(r_{o}^{2}-r_{i}^{2}\right)}$,

$v_{r z}=\frac{2 \Pi n\left(r^{3}-3\right)}{90\left(r_{o}^{2}-r_{\imath}\right)}, T=\frac{I_{z} \Pi n}{30\left(M_{h}+M_{f}\right)}$

$\Delta r=20 \mathrm{mi}_{\mathrm{i}} \quad \mathrm{I}=55 \mathrm{kgmm}^{2}, P_{\max }=1 \mathrm{MPa}, F_{\max }=1000 \mathrm{~N}$,

$T_{\max }=$ 'っ s $\mu=0.5, s=1.5, M_{s}=40 \mathrm{Nm}, M_{f}=3 \mathrm{Nm}, n=250 \mathrm{rpm}$,

$v_{s r \max }=\perp^{\prime} \mathrm{m} / \mathrm{s}, l_{\max }=30 \mathrm{~mm}, r_{i \min }=60, r_{i \max }=80, r_{o \min }=90$,

$r_{o \max }=110, t_{\min }=1.5, t_{\max }=3, F_{\min }=600, F_{\max }=1000, Z_{\min }=2, Z_{\max }=9$, 
Table 14: Comparison of results for multi-plate disc clutch brake

\begin{tabular}{lllllll}
\hline Algorithm & $r_{i}$ & $r_{0}$ & $t$ & $F$ & $Z$ & Optimal ost \\
\hline HHO & 69.9999999992493 & 90 & 1 & 1000 & 2.312781994 & $0.2597 f$ \\
TLBO [93] & 70 & 90 & 1 & 810 & 3 & 0.313656 \\
WCA [61] & 70 & 90 & 1 & 910 & 3 & $0.31,05$ \\
PVS [92] & 70 & 90 & 1 & 980 & 3 & 0.366
\end{tabular}

\subsubsection{Rolling element bearing design problem}

This engineering problem has 10 geometric variables, nine cons " "air cs considered for assembly and geometric-based restrictions and our purpose for tackling th ase to optimize (maximize) the dynamic load carrying capacity. The formulation of this te $t$ case $s$ described as follows:

Maximize $C_{d}=f_{c} Z^{2 / 3} D_{b}^{1.8} \quad$ if $D \leq 25.4 \mathrm{~mm}$

$C_{d}=3.647 f_{c} Z^{2 / 3} D_{b}^{1.4} \quad$ if $D>25.4 m m$

Subject to

$$
\begin{aligned}
& g_{1}(\vec{z})=\frac{\phi_{0}}{2 \sin ^{-1}\left(D_{b} / D_{m}\right)}-Z+1 \leq 0, \\
& g_{2}(\vec{z})=2 D_{b}-K_{D \min }(D-d)>0, \\
& g_{3}(\vec{z})=K_{D \max }(D-d)-2 D_{b} \geq 0, \\
& g_{4}(\vec{z})=\zeta B_{w}-D_{b} \leq 0, \\
& g_{5}(\vec{z})=D_{m}-0.5(D+d) \geq 0, \\
& g_{6}(\vec{z})=(0.5+e)(D+d)-D_{m_{\iota}}-n \\
& g_{7}(\vec{z})=0.5\left(D-D_{m}-D_{b}\right)-\epsilon D_{b}=0, \\
& g_{8}(\vec{z})=f_{i} \geq 0.515, \\
& g_{9}(\vec{z})=f_{o} \geq 0.515,
\end{aligned}
$$

where

$$
\begin{aligned}
& f_{c}=37.91\left[1+\left\{1 . c^{1}\left(\frac{1-\gamma}{1+\gamma}\right)^{1.72}\left(\frac{f_{i}\left(2 f_{o}-1\right)}{f_{o}\left(2 f_{i}-1\right)}\right)^{0.41}\right\}^{10 / 3}\right]^{-0.3} \\
& \left.\times\left[\frac{\gamma^{0.3}\left(1-\gamma^{\prime}\right.}{\left(1+\gamma^{\prime 1 / 3}\right.}\right\rfloor \frac{2 f_{i}}{2 f_{i}-1}\right]^{0.41} \\
& x=\left[\{(D-1) 2-3(T / 4)\}^{2}+\left\{D / 2-T / 4-D_{b}\right\}^{2}-\{d / 2+T / 4\}^{2}\right] \\
& y=2\{(\Gamma-d) / 2-3(T / 4)\}\left\{D / 2-T / 4-D_{b}\right\} \\
& \phi_{o}=2 \Pi \cdot \mathrm{nn}^{-1}\left(\frac{x}{y}\right) \\
& \gamma=\frac{D_{b}}{L_{m}}, \quad f_{i}=\frac{r_{i}}{D_{b}}, \quad f_{o}=\frac{r_{o}}{D_{b}}, \quad T=D-d-2 D_{b} \quad D=160, \quad d=90, \\
& b_{\mathrm{c}}=3 \mathrm{v}, \quad r_{i}=r_{o}=11.033 \quad 0.5(D+d) \leq D_{m} \leq 0.6(D+d), \\
& 0 .{ }^{7}(D-d) \leq D_{b} \leq 0.45(D-d), 4 \leq Z \leq 50, \quad 0.515 \leq f_{i} \text { and } f_{o} \leq 0.6 \text {, } \\
& 0.4 \leq K_{D \min } \leq 0.5 \\
& 0.6 \leq K_{D \max } \leq 0.7, \quad 0.3 \leq e \leq 0.4,0.02 \leq e \leq 0.1,0.6 \leq \zeta \leq 0.85
\end{aligned}
$$


This case covers closely $1.5 \%$ of the feasible area or he ta get space. The results of HHO is compared to GA4 [94], TLBO [93], and PVS [92] tecn iques. Table 15 tabulates the results of HHO versus those of other optimizers. From Table 1, m ou that the proposed HHO has detected the best solution with the maximum cost with a subsu ntial progress compared to GA4, TLBO, and PVS algorithms.

Table 15: Comparison of results fo. 1. "ling element bearing design problem

\begin{tabular}{|c|c|c|c|c|}
\hline Algorithms & GA4 [94] & $\overline{1 \mathrm{n}}, \overline{3]}$ & PVS [92] & $\mathrm{HHO}$ \\
\hline$D_{m}$ & 125.717100 & $12{ }^{-7191}$ & 125.719060 & 125.000000 \\
\hline$D_{b}$ & 21.423000 & 21.42559 & 21.425590 & 21.000000 \\
\hline$Z$ & $11.0000, \mathrm{~J}$ & 11.000000 & 11.000000 & 11.092073 \\
\hline$f_{i}$ & $0.515^{r} \mathrm{~J} 0$ & ( ) 515000 & 0.515000 & 0.515000 \\
\hline$f_{0}$ & 0.5150 u & ○ j15000 & 0.515000 & 0.515000 \\
\hline$K_{d \min }$ & $0 . \angle .590 n$ & J.424266 & 0.400430 & 0.400000 \\
\hline$K_{d \max }$ & ؟ & 0.633948 & 0.680160 & 0.600000 \\
\hline$\epsilon$ & $0 . x^{-143}$ & 0.300000 & 0.300000 & 0.300000 \\
\hline e & $0.0223 u$ & 0.068858 & 0.079990 & 0.050474 \\
\hline$\xi$ & 751000 & 0.799498 & 0.700000 & 0.600000 \\
\hline Maximum cost & 8184 c 30 & 81859.74 & 81859.741210 & 83011.88329 \\
\hline
\end{tabular}

\section{Discussion on res alt}

As per results in $r$ siou sections, we can recognize that the HHO shows significantly superior results for multi-di aensio al F1-F13 problems and F14-F29 test cases compared to other wellestablished optimize sur as GA, PSO, BBO, DE, CS, GWO, MFO, FPA, TLBO, BA, and FA methods. Whil the fficacy of methods such as PSO, DE, MFO, and GA significantly degrade by increasing $t_{1}$ a dim nsions, the scalability results in Fig. 12 and Table 2 expose that HHO is able to maintan a well equilibrium among the exploratory and exploitative propensities on problems top or upnies with many variables. If we observe the results of F1-F7 in Tables 3-6, there is a big, s. rnificant gap between the results of several methods such as the GA, PSO, DE, BBO, GWO, FPA, FA, and BA, with high-quality solutions found by HHO. This observation confirms the advanced exploitative merits of the proposed HHO. Based on the solution found for multimodal and hybrid composition landscapes in Table 8, we detect that HHO finds superior 
and competitive solutions based on a stable balance between the diversification and intensification inclinations and a smooth transition between the searching modes. The resu' is also support the superior exploratory strengths of the HHO. The results for six well-known ` nstrained cases in Tables 10-15 also disclose that HHO obtains the best solutions and it is one of the sop optimizers compared to many state-of-the-art techniques. The results highlight tha' the proposed HHO has several exploratory and exploitative mechanisms and consequently, it han ificiently avoided LO and immature convergence drawbacks when solving different classes of $p$ - nblems and in the case of any LO stagnation, the proposed HHO has shown a higher potf 4 tr 1 in jumping out of local optimum solutions.

The following features can theoretically assist us in realizing ‥thy $\cdots$ proposed HHO can be beneficial in exploring or exploiting the search space of a given optimı ation problem:

- Escaping energy $E$ parameter has a dynamic randor ized tine-varying nature, which can further boost the exploration and exploitation patte. $n s$ of $\mathrm{f}$ HO. This factor also requires HHO to perform a smooth transition between expl. ntior ...d exploitation.

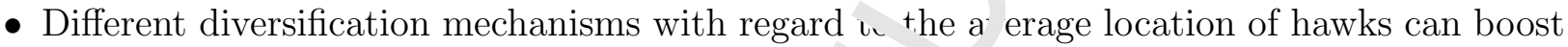
the exploratory behavior of $\mathrm{HHO}$ in initial iterat. ns.

- Different LF-based patterns with short-length ; 'mps enhance the exploitative behaviors of $\mathrm{HHO}$ when conducting a local search.

- The progressive selection scheme assists $\operatorname{arch}$ agents to progressively improve their position and only select a better position, which ca 1 - prove the quality of solutions and intensification powers of HHO during the $\operatorname{cour}_{w} v^{n}:$ it ations.

- HHO utilizes a series of searching strategies based on $E$ and $r$ parameters and then, it selects the best movement step. This c pabil y has also a constructive impact on the exploitation potential of HHO.

- The randomized jump $J \operatorname{str} n g+\wedge$ ca 1 assist candidate solutions in balancing the exploration and exploitation tendencir $s$.

- The use of adaptive and timu varying parameters allows HHO to handle difficulties of a search space includin , lo al optimal solutions, multi-modality, and deceptive optima.

\section{Conclusion and itı re irections}

In this work, a novel population-based optimization algorithm called HHO is proposed to tackle different opti nizatir $n$ tasks. The proposed HHO is inspired by the cooperative behaviors and chasing styl of puctatory birds, Harris' hawks, in nature. Several equations are designed to simulate the social ntelligence of Harris' hawks to solve optimization problems. Twenty nine unconstrained be $\cdot \mathrm{hr}$ ark problems were used to evaluate the performance of HHO. Exploitative, exploratory, mal optima avoidance of HHO was investigated using unimodal, multi-modal and compositic problems. The results obtained show that HHO was capable of finding excellent solutions compared to other well-regarded optimizers. Additionally, the results of six constrained engineering design tasks also revealed that the HHO can show superior results compared to other optimizers. 


\section{A Appendix A}

We designed the HHO as simple as possible with few exploratory and exploitative mechanisms. It is possible to utilize other evolutionary schemes such as mutation and crossr ver schemes, multiswarm and multi-leader structure, evolutionary updating structures, and char, hased phases. Such operators and ideas are beneficial for future works. In future works, the binary ana .aulti-objective versions of HHO can be developed. In addition, it can be employed to cac le various problems in engineering and other fields. Another interesting direction is to $\operatorname{com}_{\mathrm{r}}$ - $\mathrm{e}$ different constraint handling strategies in dealing with real-world constrained problems.

Table 16: Description of unimodal benchmark functic is.

\begin{tabular}{|c|c|c|c|}
\hline Function & Dimensions & 1 nnge & $f_{\min }$ \\
\hline$f_{1}(x)=\sum_{i=1}^{n} x_{i}^{2}$ & $30,100,500,100 u$ & r $-100,100]$ & 0 \\
\hline$f_{2}(x)=\sum_{i=1}^{n}\left|x_{i}\right|+\prod_{i=1}^{n}\left|x_{i}\right|$ & $30,100,500,10 u$ & {$[-10,10]$} & 0 \\
\hline$f_{3}(x)=\sum_{i=1}^{n}\left(\sum_{j-1}^{i} x_{j}\right)^{2}$ & $30,100,5$ c $\quad 1000$ & {$[-100,100]$} & 0 \\
\hline$f_{4}(x)=\max _{i}\left\{\left|x_{i}\right|, 1 \leq i \leq n\right\}$ & 30,100, bu $10 \mathrm{vu}$ & {$[-100,100]$} & 0 \\
\hline$f_{5}(x)=\sum_{i=1}^{n-1}\left[100\left(x_{i+1}-x_{i}^{2}\right)^{2}+\left(x_{i}-1\right)^{2}\right]$ & $30,1^{n n}, \cdots,-\infty j 0$ & {$[-30,30]$} & 0 \\
\hline$f_{6}(x)=\sum_{i=1}^{n}\left(\left[x_{i}+0.5\right]\right)^{2}$ & $30,100$, ๖ $\urcorner .1000$ & {$[-100,100]$} & 0 \\
\hline$f_{7}(x)=\sum_{i=1}^{n} i x_{i}^{4}+$ random $[0,1)$ & 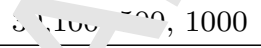 & {$[-128,128]$} & 0 \\
\hline
\end{tabular}

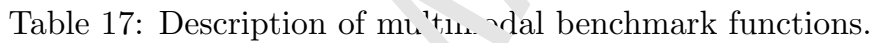

\begin{tabular}{|c|c|c|c|}
\hline Function & Dimensions & Range & $f_{\min }$ \\
\hline$f_{8}(x)=\sum_{i=1}^{n}-x_{i} \sin \left(\sqrt{\left|x_{i}\right|}\right)$ & $30,100,500,1000$ & {$[-500,500]$} & $-418.9829 \times n$ \\
\hline$f_{9}(x)=\sum_{i=1}^{n}\left[x_{i}^{2}-10 \cos \left(2 \pi x_{i}\right)+10\right]$ & $30,100,500,1000$ & {$[-5.12,5.12]$} & 0 \\
\hline$f_{10}(x)=-20 \exp \left(-0.2 \sqrt{\frac{1}{n} \sum_{i=1}^{n} x_{i}^{2}}\right)-\exp \left(\frac{1}{n} \sum_{i=\alpha_{+}}^{n} \operatorname{nos}\left(2 \pi x_{i}\right)\right)+20+e$ & $30,100,500,1000$ & {$[-32,32]$} & 0 \\
\hline$f_{11}(x)=\frac{1}{4000} \sum_{i=1}^{n} x_{i}^{2}-\prod_{i=1}^{n} \cos \left(\frac{x_{i}}{\sqrt{i}}\right)+1$ & $30,100,500,1000$ & {$[-600,600]$} & 0 \\
\hline 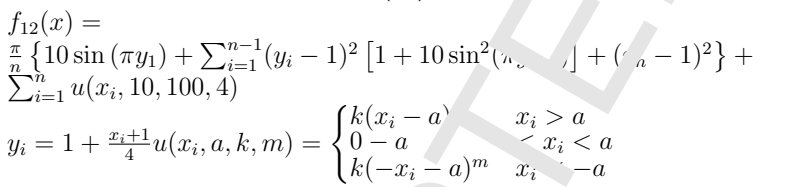 & $30,100,500,1000$ & {$[-50,50]$} & 0 \\
\hline $\begin{array}{l}f_{13}(x)= \\
0.1\left\{\sin ^{2}\left(3 \pi x_{1}\right)+\sum_{i=1}^{n}\left(x_{i}-1\right)^{2}\left\lceil+\operatorname{sir}\left(3 \pi x_{i}+1\right)\right]+\left(x_{n}-1\right)^{2}[1+\mathrm{s}\right. \\
\sum_{i=1}^{n} u\left(x_{i}, 5,100,4\right)\end{array}$ & $\begin{array}{l}30,100,500,1000 \\
\left.\left.2^{2}\left(2 \pi x_{n}\right)\right]\right\}+\end{array}$ & {$[-50,50]$} & 0 \\
\hline
\end{tabular}


Table 18: Description of fixed-dimension multimodal benchmark functions.

\begin{tabular}{|c|c|c|c|}
\hline Function & Dimensions & Range & $f_{\min }$ \\
\hline$f_{14}(x)=\left(\frac{1}{500}+\sum_{j=1}^{25} \frac{1}{j+\sum_{i=1}^{2}\left(x_{i}-a_{i j}\right)^{6}}\right)^{-1}$ & 2 & {$[-65,6$} & \\
\hline$f_{15}(x)=\sum_{i=1}^{11}\left[a_{i}-\frac{x_{1}\left(b_{i}^{2}+b_{i} x_{2}\right)}{b_{i}^{2}+b_{i} x_{3}+x_{4}}\right]^{2}$ & 4 & {$[-5,5]$} & 0.00030 \\
\hline$f_{16}(x)=4 x_{1}^{2}-2.1 x_{1}^{4}+\frac{1}{3} x_{1}^{6}+x_{1} x_{2}-4 x_{2}^{2}+4 x_{2}^{4}$ & 2 & {$\left[\begin{array}{ll}-5 & ,\end{array}\right]$} & -1.0316 \\
\hline $\begin{array}{l}f_{17}(x)=\left(x_{2}-\frac{5.1}{4 \pi^{2}} x_{1}^{2}+\frac{5}{\pi} x_{1}-6\right)^{2}+10\left(1-\frac{1}{8 \pi}\right) \cos x_{1}+10 \\
f_{18}(x)= \\
{\left[1+\left(x_{1}+x_{2}+1\right)^{2}\left(19-14 x_{1}+3 x_{1}^{2}-14 x_{2}+6 x_{1} x_{2}+3 x_{2}^{2}\right)\right]} \\
\times\left[30+\left(2 x_{1}-3 x_{2}\right)^{2} \times\left(18-32 x_{1}+12 x_{1}^{2}+48 x_{2}-36 x_{1} x_{2}+27 x_{2}^{2}\right)\right]\end{array}$ & $\begin{array}{l}2 \\
2\end{array}$ & & 0.398 \\
\hline$f_{19}(x)=-\sum_{i=1}^{4} c_{i} \exp \left(-\sum_{j=1}^{3} a_{i j}\left(x_{j}-p_{i j}\right)^{2}\right)$ & 3 & {$\left[\begin{array}{ll}1 & 3\end{array}\right]$} & -3.86 \\
\hline$f_{20}(x)=-\sum_{i=1}^{4} c_{i} \exp \left(-\sum_{j=1}^{6} a_{i j}\left(x_{j}-p_{i j}\right)^{2}\right)$ & 6 & {$\left[6,{ }^{-1}\right]$} & -3.32 \\
\hline$f_{21}(x)=-\sum_{i=1}^{5}\left[\left(X-a_{i}\right)\left(X-a_{i}\right)^{T}+c_{i}\right]^{-1}$ & 4 & L 10$]$ & -10.1532 \\
\hline$f_{22}(x)=-\sum_{i=1}^{7}\left[\left(X-a_{i}\right)\left(X-a_{i}\right)^{T}+c_{i}\right]^{-1}$ & 4 & $r, 10]$ & -10.4028 \\
\hline$f_{23}(x)=-\sum_{i=1}^{10}\left[\left(X-a_{i}\right)\left(X-a_{i}\right)^{T}+c_{i}\right]^{-1}$ & & {$[0.10]$} & -10.5363 \\
\hline
\end{tabular}

Table 19: Details of hybrid composition functions F24-F29 (MM: Mי1ltı-moda', R: Rotated, NS: Non-Separable, S: Scalable, D: Dimension)

\begin{tabular}{|c|c|c|c|c|}
\hline ID (CEC5-ID) & Description & Properties & D & Range \\
\hline F24 (C16) & Rotated Hybrid Composition Function & MM, R, NS, S & 30 & {$[-5,5]^{D}$} \\
\hline $\mathrm{F} 25(\mathrm{C} 18)$ & Rotated Hybrid Composition Function & MM, R, NS, S & 30 & {$[-5,5]^{D}$} \\
\hline F26 (C19) & Rotated Hybrid Composition Function with narr vawin gruval optimum & MM, NS, S & 30 & {$[-5,5]^{D}$} \\
\hline $\mathrm{F} 27(\mathrm{C} 20)$ & Rotated Hybrid Composition Function with Global $\iota_{1}{ }^{2}$ imum on the Bounds & MM, NS, S & 30 & {$[-5,5]^{D}$} \\
\hline $\mathrm{F} 28(\mathrm{C} 21)$ & Rotated Hybrid Composition Function & MM, R, NS, S & 30 & {$[-5,5]^{D}$} \\
\hline F29 (C25) & Rotated Hybrid Composition Function withou bous w & MM, NS, S & 30 & {$[-5,5]^{D}$} \\
\hline
\end{tabular}

\section{B Appendix B}

Table 20: p-values of the Wilcoxon rank-sum test with $5 \%$ significance for F1-F13 with 30 dimensions (p-values $\geq$ 0.05 are shown in bold face, NaN means "Nc, a Nu nber" returned by the test)

\begin{tabular}{|c|c|c|c|c|c|c|c|c|c|c|c|}
\hline & GA & PSO & $\mathrm{BBO}$ & FPA & FWC & BAT & FA & $\mathrm{CS}$ & MFO & TLBO & $\mathrm{DE}$ \\
\hline F1 & $2.85 \mathrm{E}-11$ & $2.88 \mathrm{E}-11$ & $2.52 \mathrm{E}-11$ & $\bar{l} \sqrt{2 \mathrm{E}-11}$ & $3.4-11$ & $3.02 \mathrm{E}-11$ & $3.02 \mathrm{E}-11$ & $3.02 \mathrm{E}-11$ & $3.02 \mathrm{E}-11$ & $3.02 \mathrm{E}-11$ & $3.02 \mathrm{E}-11$ \\
\hline F2 & $2.72 \mathrm{E}-11$ & $2.52 \mathrm{E}-11$ & $4.56 \mathrm{E}-11$ & $3.02 \mathrm{~F} \quad 1$ & 3 ก $2 \mathrm{E}-11$ & $3.02 \mathrm{E}-11$ & $3.02 \mathrm{E}-11$ & $3.02 \mathrm{E}-11$ & $3.02 \mathrm{E}-11$ & $3.02 \mathrm{E}-11$ & $3.02 \mathrm{E}-11$ \\
\hline F3 & $2.71 \mathrm{E}-11$ & $2.63 \mathrm{E}-11$ & $2.79 \mathrm{E}-11$ & n -11 & $.02 \mathrm{E}-11$ & $3.02 \mathrm{E}-11$ & $3.02 \mathrm{E}-11$ & $3.02 \mathrm{E}-11$ & $3.02 \mathrm{E}-11$ & $3.02 \mathrm{E}-11$ & $3.02 \mathrm{E}-11$ \\
\hline $\mathrm{F} 4$ & $2.62 \mathrm{E}-11$ & $2.84 \mathrm{E}-11$ & $2.62 \mathrm{E}-1$ & $3.0<-11$ & $3.02 \mathrm{E}-11$ & $3.02 \mathrm{E}-11$ & $3.02 \mathrm{E}-11$ & $3.02 \mathrm{E}-11$ & $3.02 \mathrm{E}-11$ & $3.02 \mathrm{E}-11$ & $3.02 \mathrm{E}-11$ \\
\hline F5 & $2.62 \mathrm{E}-11$ & $2.52 \mathrm{E}-11$ & $2.72 \mathrm{~F} \quad 11$ & $3.02 \mathrm{E}-\mathrm{l}_{\perp}$ & $3.02 \mathrm{E}-11$ & $3.02 \mathrm{E}-11$ & $3.02 \mathrm{E}-11$ & $3.02 \mathrm{E}-11$ & $3.02 \mathrm{E}-11$ & $3.02 \mathrm{E}-11$ & $3.02 \mathrm{E}-11$ \\
\hline F6 & $2.72 \mathrm{E}-11$ & $2.71 \mathrm{E}-11$ & $2.6^{\sigma}-I_{\perp}$ & ?.02E-11 & 3.02E-11 & $3.02 \mathrm{E}-11$ & $3.02 \mathrm{E}-11$ & $3.02 \mathrm{E}-11$ & $3.02 \mathrm{E}-11$ & $2.25 \mathrm{E}-04$ & $3.02 \mathrm{E}-11$ \\
\hline F7 & $2.52 \mathrm{E}-11$ & $2.71 \mathrm{E}-11$ & $9.19 \mathrm{E}-11$ & 3.. ${ }^{\mathrm{T}}-11$ & $3.69 \mathrm{E}-11$ & $3.02 \mathrm{E}-11$ & $3.02 \mathrm{E}-11$ & $3.02 \mathrm{E}-11$ & $3.02 \mathrm{E}-11$ & $3.02 \mathrm{E}-11$ & $3.02 \mathrm{E}-11$ \\
\hline F8 & 7.83E-09 & $2.71 \mathrm{E}-11$ & ᄃ 09 & $3.02 \mathrm{E}-11$ & $3.02 \mathrm{E}-11$ & $3.02 \mathrm{E}-11$ & $3.02 \mathrm{E}-11$ & $3.02 \mathrm{E}-11$ & $3.02 \mathrm{E}-11$ & $3.02 \mathrm{E}-11$ & $3.02 \mathrm{E}-11$ \\
\hline F9 & $9.49 \mathrm{E}-13$ & $1.00 \mathrm{E}-12$ & $\mathrm{NaN}$ & $1.21 \mathrm{E}-12$ & $4.35 \mathrm{E}-12$ & $1.21 \mathrm{E}-12$ & $1.21 \mathrm{E}-12$ & $1.21 \mathrm{E}-12$ & $1.21 \mathrm{E}-12$ & $4.57 \mathrm{E}-12$ & $1.21 \mathrm{E}-12$ \\
\hline F10 & $1.01 \mathrm{E}-12$ & $1.14 \mathrm{E}-1 \mathrm{c}$ & $1.05,-12$ & $1.21 \mathrm{E}-12$ & $1.16 \mathrm{E}-12$ & $1.21 \mathrm{E}-12$ & $1.21 \mathrm{E}-12$ & $1.21 \mathrm{E}-12$ & $1.21 \mathrm{E}-12$ & $4.46 \mathrm{E}-13$ & $1.21 \mathrm{E}-12$ \\
\hline F11 & $9.53 \mathrm{E}-13$ & $9.57 \mathrm{E}-13$ & y. $=-13$ & $1.21 \mathrm{E}-12$ & $2.79 \mathrm{E}-03$ & $1.21 \mathrm{E}-12$ & $1.21 \mathrm{E}-12$ & $1.21 \mathrm{E}-12$ & $1.21 \mathrm{E}-12$ & $\mathrm{NaN}$ & $1.21 \mathrm{E}-12$ \\
\hline F12 & $2.63 \mathrm{E}-11$ & $2.51^{\top} \quad 11$ & ?. $63 \mathrm{E}-\perp$ & $3.02 \mathrm{E}-11$ & $3.02 \mathrm{E}-11$ & $3.02 \mathrm{E}-11$ & $3.02 \mathrm{E}-11$ & $1.01 \mathrm{E}-08$ & $3.02 \mathrm{E}-11$ & $1.07 \mathrm{E}-06$ & $3.02 \mathrm{E}-11$ \\
\hline F13 & $2.51 \mathrm{E}-11$ & 2.7 ¿-11 & $2.61 \mathrm{E} 11$ & $3.02 \mathrm{E}-11$ & $3.02 \mathrm{E}-11$ & 3.02E-11 & $5.49 \mathrm{E}-11$ & $3.02 \mathrm{E}-11$ & $3.02 \mathrm{E}-11$ & $2.00 \mathrm{E}-06$ & $3.02 \mathrm{E}-11$ \\
\hline
\end{tabular}


Table 21: p-values of the Wilcoxon rank-sum test with 5\% significance for F1-F13 with 100 dimensions (p-values $\geq 0.05$ are shown in bold face)

\begin{tabular}{|c|c|c|c|c|c|c|c|c|c|c|c|}
\hline & GA & PSO & $\mathrm{BBO}$ & FPA & GWO & BAT & FA & CS & MFO & $\overline{\mathrm{TL}^{\prime}} \cup \overline{ }$ & $\mathrm{DE}$ \\
\hline F1 & $2.98 \mathrm{E}-11$ & $2.52 \mathrm{E}-11$ & $2.52 \mathrm{E}-11$ & $3.01 \mathrm{E}-11$ & $3.02 \mathrm{E}-11$ & $3.02 \mathrm{E}-11$ & $3.02 \mathrm{E}-11$ & $3.02 \mathrm{E}-11$ & $3.02 \mathrm{E}-11$ & $3.62 \overline{\mathrm{E}-11}$ & $72 \mathrm{E}-11$ \\
\hline F2 & $2.88 \mathrm{E}-11$ & $2.72 \mathrm{E}-11$ & $2.72 \mathrm{E}-11$ & $3.02 \mathrm{E}-11$ & $3.02 \mathrm{E}-11$ & $3.02 \mathrm{E}-11$ & $3.02 \mathrm{E}-11$ & $3.02 \mathrm{E}-11$ & $3.02 \mathrm{E}-11$ & $\cdots \mathrm{F}_{-11}$ & $3.02 \mathrm{E}-11$ \\
\hline F3 & $2.72 \mathrm{E}-11$ & $2.72 \mathrm{E}-11$ & $2.52 \mathrm{E}-11$ & $3.02 \mathrm{E}-11$ & $3.02 \mathrm{E}-11$ & $3.02 \mathrm{E}-11$ & $3.02 \mathrm{E}-11$ & $3.02 \mathrm{E}-11$ & $3.02 \mathrm{E}-11$ & $3.02 \Vdash 11$ & $3.02 \mathrm{E}-11$ \\
\hline F4 & $2.40 \mathrm{E}-11$ & $2.52 \mathrm{E}-11$ & $2.51 \mathrm{E}-11$ & $3.02 \mathrm{E}-11$ & $3.02 \mathrm{E}-11$ & $3.02 \mathrm{E}-11$ & $3.02 \mathrm{E}-11$ & $3.02 \mathrm{E}-11$ & $3.02 \mathrm{E}-1$ & $3.01-11$ & $3.02 \mathrm{E}-11$ \\
\hline F5 & $2.72 \mathrm{E}-11$ & $2.62 \mathrm{E}-11$ & $2.84 \mathrm{E}-11$ & $3.02 \mathrm{E}-11$ & $3.02 \mathrm{E}-11$ & $3.02 \mathrm{E}-11$ & $3.02 \mathrm{E}-11$ & $3.02 \mathrm{E}-11$ & $3.02 \mathrm{E}-11$ & E-11 & $3.02 \mathrm{E}-11$ \\
\hline F6 & $2.52 \mathrm{E}-11$ & $2.52 \mathrm{E}-11$ & $2.52 \mathrm{E}-11$ & $3.02 \mathrm{E}-11$ & $3.02 \mathrm{E}-11$ & $3.02 \mathrm{E}-11$ & $3.02 \mathrm{E}-11$ & $3.02 \mathrm{E}-11$ & $3.02 \mathrm{E}-$. & $3.02 \mathrm{~L}^{-1}$ & $3.02 \mathrm{E}-11$ \\
\hline F7 & $2.71 \mathrm{E}-11$ & $2.79 \mathrm{E}-11$ & $2.52 \mathrm{E}-11$ & $3.02 \mathrm{E}-11$ & $3.02 \mathrm{E}-11$ & $3.02 \mathrm{E}-11$ & $3.02 \mathrm{E}-11$ & $3.02 \mathrm{E}-11$ & $3.02 \mathrm{~F}-11$ & $\therefore$ ` & $3.02 \mathrm{E}-11$ \\
\hline F8 & $2.72 \mathrm{E}-11$ & $2.51 \mathrm{E}-11$ & $2.83 \mathrm{E}-11$ & $3.02 \mathrm{E}-11$ & $3.02 \mathrm{E}-11$ & $3.02 \mathrm{E}-11$ & $3.02 \mathrm{E}-11$ & $5.57 \mathrm{E}-10$ & $3,2 \mathrm{E}-1$ & $3.02 \mathrm{~L}-11$ & $3.02 \mathrm{E}-11$ \\
\hline F9 & $1.06 \mathrm{E}-12$ & $9.57 \mathrm{E}-13$ & $9.54 \mathrm{E}-13$ & $1.21 \mathrm{E}-12$ & $1.21 \mathrm{E}-12$ & $1.21 \mathrm{E}-12$ & $1.21 \mathrm{E}-12$ & $1.21 \mathrm{E}-12$ & $.21 \mathrm{E}-$ & ? $1 \mathrm{E}-01$ & $1.21 \mathrm{E}-12$ \\
\hline F10 & $9.56 \mathrm{E}-13$ & $9.57 \mathrm{E}-13$ & $1.09 \mathrm{E}-12$ & $1.21 \mathrm{E}-12$ & $1.21 \mathrm{E}-12$ & $1.21 \mathrm{E}-12$ & $1.21 \mathrm{E}-12$ & $1.21 \mathrm{E}-12$ & ?1F 12 & $4.16 \mathrm{E}-14$ & $1.21 \mathrm{E}-12$ \\
\hline F11 & $1.06 \mathrm{E}-12$ & $9.55 \mathrm{E}-13$ & $9.56 \mathrm{E}-13$ & $1.21 \mathrm{E}-12$ & $1.21 \mathrm{E}-12$ & $1.21 \mathrm{E}-12$ & $1.21 \mathrm{E}-12$ & $1.21 \mathrm{E}-12$ & $1.2 \pm .12$ & $\mathrm{NaN}$ & $1.21 \mathrm{E}-12$ \\
\hline F12 & $2.72 \mathrm{E}-11$ & $2.52 \mathrm{E}-11$ & $2.52 \mathrm{E}-11$ & $3.02 \mathrm{E}-11$ & $3.02 \mathrm{E}-11$ & $3.02 \mathrm{E}-11$ & $3.02 \mathrm{E}-11$ & $3.02 \mathrm{~F}-1$ & $3.02 \mathrm{E}-1 \perp$ & $3.02 \mathrm{E}-11$ & $3.02 \mathrm{E}-11$ \\
\hline F13 & $2.72 \mathrm{E}-11$ & $2.72 \mathrm{E}-11$ & $2.52 \mathrm{E}-11$ & $3.02 \mathrm{E}-11$ & $3.02 \mathrm{E}-11$ & $3.02 \mathrm{E}-11$ & $3.02 \mathrm{E}-11$ & $3.02-11$ & $3.02 \times 11$ & $3.02 \mathrm{E}-11$ & $3.02 \mathrm{E}-11$ \\
\hline
\end{tabular}

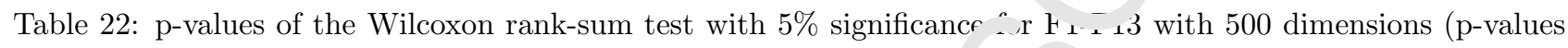
$\geq 0.05$ are shown in bold face)

\begin{tabular}{|c|c|c|c|c|c|c|c|c|c|c|c|}
\hline & GA & PSO & $\mathrm{BBO}$ & FPA & GWO & BAT & FA & $\mathrm{CS}$ & $\mathrm{MFO}$ & TLBO & $\mathrm{DE}$ \\
\hline F1 & $2.94 \mathrm{E}-11$ & $2.79 \mathrm{E}-11$ & $2.72 \mathrm{E}-11$ & $3.02 \mathrm{E}-11$ & $3.02 \mathrm{E}-11$ & $3.02 \mathrm{E}-11$ & $3.02 \mathrm{E}-11$ & 72E-11 & $3.02 \mathrm{E}-11$ & $3.02 \mathrm{E}-11$ & $3.02 \mathrm{E}-11$ \\
\hline F2 & $2.52 \mathrm{E}-11$ & $2.63 \mathrm{E}-11$ & $2.52 \mathrm{E}-11$ & $3.02 \mathrm{E}-11$ & $3.02 \mathrm{E}-11$ & $3.02 \mathrm{E}-11$ & ? $\cap 2 \mathrm{E}-11$ & 3.0 E-11 & $3.02 \mathrm{E}-11$ & $3.02 \mathrm{E}-11$ & $3.02 \mathrm{E}-11$ \\
\hline F3 & $2.88 \mathrm{E}-11$ & $2.52 \mathrm{E}-11$ & $2.72 \mathrm{E}-11$ & $3.02 \mathrm{E}-11$ & $3.02 \mathrm{E}-11$ & $3.02 \mathrm{E}-11$ & $3 . U_{2} \quad 11$ & 3.r.E-11 & $3.02 \mathrm{E}-11$ & $3.02 \mathrm{E}-11$ & $3.02 \mathrm{E}-11$ \\
\hline F4 & $2.25 \mathrm{E}-11$ & $2.52 \mathrm{E}-11$ & $2.59 \mathrm{E}-11$ & 3.02E-11 & $3.02 \mathrm{E}-11$ & $3.02 \mathrm{E}-11$ & ${ }^{2} 2 \mathrm{E}-\mathrm{I}_{\perp}$ & $.02 \mathrm{E}-11$ & $3.02 \mathrm{E}-11$ & 3.02E-11 & $3.02 \mathrm{E}-11$ \\
\hline F5 & $2.72 \mathrm{E}-11$ & $2.72 \mathrm{E}-11$ & $2.72 \mathrm{E}-11$ & $3.02 \mathrm{E}-11$ & $3.02 \mathrm{E}-11$ & $3.02 \mathrm{E}-11$ & $3.0 \angle \alpha_{2} \quad 11$ & $3.02 \mathrm{E}-11$ & 3.02E-11 & $3.02 \mathrm{E}-11$ & $3.02 \mathrm{E}-11$ \\
\hline F6 & $2.52 \mathrm{E}-11$ & $2.52 \mathrm{E}-11$ & $2.52 \mathrm{E}-11$ & $3.02 \mathrm{E}-11$ & $3.02 \mathrm{E}-11$ & $3.02 \mathrm{~F}^{+1}$ & & $3.02 \mathrm{E}-11$ & $3.02 \mathrm{E}-11$ & $3.02 \mathrm{E}-11$ & $3.02 \mathrm{E}-11$ \\
\hline F7 & $2.52 \mathrm{E}-11$ & $2.79 \mathrm{E}-11$ & $2.52 \mathrm{E}-11$ & $3.02 \mathrm{E}-11$ & 3.02E-11 & $3.02 \mathrm{E}-1$ & $3.02 \mathrm{E}-11$ & $3.02 \mathrm{E}-11$ & $3.02 \mathrm{E}-11$ & $4.98 \mathrm{E}-11$ & $3.02 \mathrm{E}-11$ \\
\hline F8 & $2.52 \mathrm{E}-11$ & $2.72 \mathrm{E}-11$ & $2.63 \mathrm{E}-11$ & $3.02 \mathrm{E}-11$ & $3.02 \mathrm{E}-11$ & $3.02 \mathrm{E}-11$ & o. ${ }^{\wedge} \mathrm{F}_{\mathrm{j}}-11$ & $3.02 \mathrm{E}-11$ & $3.02 \mathrm{E}-11$ & $3.02 \mathrm{E}-11$ & $3.02 \mathrm{E}-11$ \\
\hline F9 & $1.06 \mathrm{E}-12$ & $1.06 \mathrm{E}-12$ & $1.06 \mathrm{E}-12$ & $1.21 \mathrm{E}-12$ & $1.21 \mathrm{E}-12$ & & $121 \mathrm{E}-12$ & $1.21 \mathrm{E}-12$ & $1.21 \mathrm{E}-12$ & $\mathrm{NaN}$ & $1.21 \mathrm{E}-12$ \\
\hline F10 & $9.57 \mathrm{E}-13$ & $9.57 \mathrm{E}-13$ & $1.06 \mathrm{E}-12$ & $1.21 \mathrm{E}-12$ & $1.21 \mathrm{E}-12$ & $1 . \angle \quad \mathrm{E}-11^{r}$ & $1.21 \mathrm{E}-12$ & $1.21 \mathrm{E}-12$ & $1.21 \mathrm{E}-12$ & $6.14 \mathrm{E}-14$ & $1.21 \mathrm{E}-12$ \\
\hline F11 & $9.57 \mathrm{E}-13$ & $9.57 \mathrm{E}-13$ & $1.06 \mathrm{E}-12$ & $1.21 \mathrm{E}-12$ & $1.21 \mathrm{E}-1 ?$ & $1.21 \mathrm{~L} \quad 2$ & $1.21 \mathrm{E}-12$ & $1.21 \mathrm{E}-12$ & $1.21 \mathrm{E}-12$ & $\mathrm{NaN}$ & $1.21 \mathrm{E}-12$ \\
\hline F12 & $2.52 \mathrm{E}-11$ & $2.52 \mathrm{E}-11$ & $2.79 \mathrm{E}-11$ & 3.02E-11 & $3.02 \mathrm{E}-1$ & n2E-1 & $3.02 \mathrm{E}-11$ & $3.02 \mathrm{E}-11$ & $3.02 \mathrm{E}-11$ & $3.02 \mathrm{E}-11$ & $3.02 \mathrm{E}-11$ \\
\hline F13 & $2.79 \mathrm{E}-11$ & $2.52 \mathrm{E}-11$ & $2.72 \mathrm{E}-11$ & $3.02 \mathrm{E}-11$ & $3.02 \mathrm{E}-11$ & $3.0<-11$ & $3.02 \mathrm{E}-11$ & $3.02 \mathrm{E}-11$ & $3.02 \mathrm{E}-11$ & $3.02 \mathrm{E}-11$ & $3.02 \mathrm{E}-11$ \\
\hline
\end{tabular}

Table 23: p-values of the Wilcoxon rank-sum test $\mathrm{w}_{2}$ h 5\% significance for F1-F13 with 1000 dimensions (p-values $\geq 0.05$ are shown in bold face)

\begin{tabular}{|c|c|c|c|c|c|c|c|c|c|c|c|}
\hline & GA & PSO & $\mathrm{BBO}$ & FPA & GWe & BAT & FA & $\mathrm{CS}$ & MFO & TLBO & $\mathrm{DE}$ \\
\hline F1 & $3.01 \mathrm{E}-11$ & $2.52 \mathrm{E}-11$ & $2.52 \mathrm{E}-11$ & $\overline{3.02}, \overline{11}$ & $3.02 \mathrm{E} \cdot \overline{1}$ & $3.02 \mathrm{E}-11$ & $3.02 \mathrm{E}-11$ & $3.02 \mathrm{E}-11$ & $3.02 \mathrm{E}-11$ & $3.02 \mathrm{E}-11$ & $3.02 \mathrm{E}-11$ \\
\hline $\mathrm{F} 2$ & $2.63 \mathrm{E}-11$ & $1.21 \mathrm{E}-12$ & $2.72 \mathrm{E}-11$ & 3. ${ }^{n} 2 \mathrm{E}-1_{\perp}$ & $202 F \quad 11$ & $1.21 \mathrm{E}-12$ & $1.21 \mathrm{E}-12$ & $3.02 \mathrm{E}-11$ & $1.21 \mathrm{E}-12$ & $1.21 \mathrm{E}-12$ & $1.21 \mathrm{E}-12$ \\
\hline F3 & $2.86 \mathrm{E}-11$ & $2.52 \mathrm{E}-11$ & $2.52 \mathrm{E}-11$ & J $2 \mathrm{E}-11$ & 3.c. & $3.02 \mathrm{E}-11$ & $3.02 \mathrm{E}-11$ & $3.02 \mathrm{E}-11$ & $3.02 \mathrm{E}-11$ & $3.02 \mathrm{E}-11$ & $3.02 \mathrm{E}-11$ \\
\hline F4 & $1.93 \mathrm{E}-11$ & $2.52 \mathrm{E}-11$ & $2.07 \mathrm{E}-11$ & $3.02 \mathrm{~F} \quad 1$ & 3 ก2E-11 & $3.02 \mathrm{E}-11$ & $3.02 \mathrm{E}-11$ & $3.02 \mathrm{E}-11$ & $3.02 \mathrm{E}-11$ & $3.02 \mathrm{E}-11$ & $3.02 \mathrm{E}-11$ \\
\hline F5 & $2.72 \mathrm{E}-11$ & $2.52 \mathrm{E}-11$ & $2.52 \mathrm{E}-11$ & - $2-11$ & $.02 \mathrm{E}-11$ & $3.02 \mathrm{E}-11$ & $3.02 \mathrm{E}-11$ & $3.02 \mathrm{E}$ & $3.02 \mathrm{E}-11$ & $3.02 \mathrm{E}-11$ & 3.02 \\
\hline F6 & $2.63 \mathrm{E}-11$ & $2.63 \mathrm{E}-11$ & $2.63 \mathrm{E}-1$ & $3.0<-11$ & $3.02 \mathrm{E}-11$ & $3.02 \mathrm{E}-11$ & $3.02 \mathrm{E}-11$ & $3.02 \mathrm{E}-11$ & $3.02 \mathrm{E}-11$ & $3.02 \mathrm{E}-11$ & $3.02 \mathrm{E}-11$ \\
\hline F7 & $2.63 \mathrm{E}-11$ & $2.52 \mathrm{E}-11$ & $2.52 \mathrm{~F} \quad 11$ & $3.02 \mathrm{E}-\mathrm{l}_{\perp}$ & $3.02 \mathrm{E}-11$ & $3.02 \mathrm{E}-11$ & $3.02 \mathrm{E}-11$ & $3.02 \mathrm{E}-11$ & $3.02 \mathrm{E}-11$ & $3.02 \mathrm{E}-11$ & $3.02 \mathrm{E}-11$ \\
\hline $\mathrm{F} 8$ & $2.52 \mathrm{E}-11$ & $2.52 \mathrm{E}-11$ & $2.5-\mathrm{I}_{\perp}$ & 3.02E-11 & $3.02 \mathrm{E}-11$ & $3.02 \mathrm{E}-11$ & $3.02 \mathrm{E}-11$ & $3.02 \mathrm{E}$ & $3.02 \mathrm{E}-11$ & $3.02 \mathrm{E}-11$ & 3.02 \\
\hline F9 & $1.01 \mathrm{E}-12$ & $1.06 \mathrm{E}-12$ & $9.5 / \mathrm{E}-13$ & 1.2 "-12 & $1.21 \mathrm{E}-12$ & $1.21 \mathrm{E}-12$ & $1.21 \mathrm{E}-12$ & $1.21 \mathrm{E}$ & $1.21 \mathrm{E}-12$ & $\mathrm{NaN}$ & $1.21 \mathrm{E}-12$ \\
\hline F10 & $1.01 \mathrm{E}-12$ & $1.01 \mathrm{E}-12$ & $r \quad \ldots \quad 13$ & $1.21 \mathrm{E}-12$ & $1.21 \mathrm{E}-12$ & $1.21 \mathrm{E}-12$ & $1.21 \mathrm{E}-12$ & $1.21 \mathrm{E}-12$ & $1.21 \mathrm{E}-12$ & $8.72 \mathrm{E}-14$ & $1.21 \mathrm{E}-12$ \\
\hline F11 & $1.06 \mathrm{E}-12$ & $1.01 \mathrm{E}-12$ & $9.57 \mathrm{~F} \quad 13$ & $1.21 \mathrm{E}-12$ & $1.21 \mathrm{E}-12$ & $1.21 \mathrm{E}-12$ & $1.21 \mathrm{E}-12$ & $1.21 \mathrm{E}-12$ & $1.21 \mathrm{E}-12$ & $1.17 \mathrm{E}-13$ & $1.21 \mathrm{E}-12$ \\
\hline $\mathrm{F} 12$ & $2.52 \mathrm{E}-11$ & $2.52 \mathrm{E}-1 \mathrm{i}$ & ?. $72,-11$ & $3.02 \mathrm{E}-11$ & $3.02 \mathrm{E}-11$ & $3.02 \mathrm{E}-11$ & $3.02 \mathrm{E}-11$ & $3.02 \mathrm{E}-11$ & $3.02 \mathrm{E}-11$ & $3.02 \mathrm{E}-11$ & $3.02 \mathrm{E}-11$ \\
\hline F13 & $2.52 \mathrm{E}-11$ & $2.63 \mathrm{E}-11$ & 2.. ${ }^{2}-11$ & $3.02 \mathrm{E}-11$ & $3.02 \mathrm{E}-11$ & $3.02 \mathrm{E}-11$ & $3.02 \mathrm{E}-11$ & $3.02 \mathrm{E}-11$ & $3.02 \mathrm{E}-11$ & $3.02 \mathrm{E}-11$ & $3.02 \mathrm{E}-11$ \\
\hline
\end{tabular}

Table 24: p-values of the : ${ }^{\text {illcr }}$ xon ank-sum test with $5 \%$ significance for F14-F29 problems(p-values $\geq 0.05$ are shown in bold face)

\begin{tabular}{|c|c|c|c|c|c|c|c|c|c|c|c|}
\hline & GA & $\mathrm{PSO}$ & $\mathrm{BBO}$ & FPA & GWO & BAT & FA & CS & MFO & TLBO & $\mathrm{DE}$ \\
\hline F14 & $8.15 \mathrm{E}-02$ & ?.89E-08 & $8.15 \mathrm{E}-03$ & $1.08 \mathrm{E}-01$ & $5.20 \mathrm{E}-08$ & $7.46 \mathrm{E}-12$ & $1.53 \mathrm{E}-09$ & $6.13 \mathrm{E}-14$ & $9.42 \mathrm{E}-06$ & $8.15 \mathrm{E}-02$ & $1.00 \mathrm{E}+00$ \\
\hline F15 & $2.78 \mathrm{E}-11$ & 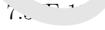 & $2.51 \mathrm{E}-11$ & $9.76 \mathrm{E}-10$ & $1.37 \mathrm{E}-01$ & $3.34 \mathrm{E}-11$ & $3.16 \mathrm{E}-10$ & $8.69 \mathrm{E}-10$ & $5.00 \mathrm{E}-10$ & $5.08 \mathrm{E}-06$ & $3.92 \mathrm{E}-02$ \\
\hline F16 & $1.0^{r}-12$ & 9. $53 \mathrm{E}-13$ & $9.49 \mathrm{E}-13$ & $\mathrm{NaN}$ & $\mathrm{NaN}$ & $5.54 \mathrm{E}-03$ & $\mathrm{NaN}$ & $\mathrm{NaN}$ & $\mathrm{NaN}$ & $\mathrm{NaN}$ & $\mathrm{NaN}$ \\
\hline F17 & 1. $\mathrm{E}$ E-12 & 1.8 $\mathrm{E}-12$ & $2.06 \mathrm{E}-12$ & $1.61 \mathrm{E}-01$ & $1.61 \mathrm{E}-01$ & $5.97 \mathrm{E}-01$ & $1.61 \mathrm{E}-01$ & $1.61 \mathrm{E}-01$ & $1.61 \mathrm{E}-01$ & $1.61 \mathrm{E}-01$ & $1.61 \mathrm{E}-01$ \\
\hline F18 & $\mathrm{Na}$ & $9.5:-13$ & $\mathrm{NaN}$ & $\mathrm{NaN}$ & $1.09 \mathrm{E}-02$ & $1.34 \mathrm{E}-03$ & $\mathrm{NaN}$ & $\mathrm{NaN}$ & $\mathrm{NaN}$ & $\mathrm{NaN}$ & $\mathrm{NaN}$ \\
\hline F19 & $2.50 \_\cdot 1$ & $5 \quad 4 \mathrm{E}-02$ & $1.91 \mathrm{E}-09$ & $1.65 \mathrm{E}-11$ & $1.06 \mathrm{E}-01$ & $5.02 \mathrm{E}-10$ & $1.65 \mathrm{E}-11$ & $1.65 \mathrm{E}-11$ & $4.54 \mathrm{E}-10$ & $1.65 \mathrm{E}-11$ & 1.65E-11 \\
\hline $\mathrm{F} 2 \mathrm{r}$ & $8.74 \mathrm{E}-03$ & $2.54 \mathrm{E}-04$ & $8.15 \mathrm{E}-03$ & $6.15 \mathrm{E}-03$ & $5.74 \mathrm{E}-06$ & $5.09 \mathrm{E}-06$ & $1.73 \mathrm{E}-07$ & $\mathrm{NaN}$ & $1.73 \mathrm{E}-04$ & $1.73 \mathrm{E}-04$ & $1.73 \mathrm{E}-04$ \\
\hline F2. & $1 . \angle \angle L$ & $6.25 \mathrm{E}-05$ & $5.54 \mathrm{E}-03$ & $1.91 \mathrm{E}-08$ & $5.54 \mathrm{E}-03$ & $6.85 \mathrm{E}-07$ & $1.71 \mathrm{E}-07$ & $1.91 \mathrm{E}-08$ & $9.42 \mathrm{E}-06$ & $1.73 \mathrm{E}-04$ & $1.79 \mathrm{E}-04$ \\
\hline $\mathrm{F} 22$ & $6^{4}-07$ & $5.00 \mathrm{E}-10$ & $8.15 \mathrm{E}-08$ & $2.51 \mathrm{E}-11$ & $8.15 \mathrm{E}-08$ & $6.63 \mathrm{E}-07$ & $5.24 \mathrm{E}-04$ & $1.73 \mathrm{E}-08$ & $8.15 \mathrm{E}-08$ & $8.81 \mathrm{E}-10$ & $1.21 \mathrm{E}-12$ \\
\hline F23 & 1. E-05 & $5.00 \mathrm{E}-10$ & $8.88 \mathrm{E}-08$ & $2.51 \mathrm{E}-11$ & $8.88 \mathrm{E}-08$ & $1.73 \mathrm{E}-08$ & $5.14 \mathrm{E}-04$ & $1.69 \mathrm{E}-08$ & $8.88 \mathrm{E}-08$ & $8.81 \mathrm{E}-10$ & $\mathrm{NaN}$ \\
\hline F24 & $2.4 \mathrm{~L}-01$ & $4.69 \mathrm{E}-08$ & $1.64 \mathrm{E}-05$ & $1.17 \mathrm{E}-05$ & $2.84 \mathrm{E}-04$ & $3.02 \mathrm{E}-11$ & 3.03E-03 & $3.08 \mathrm{E}-08$ & $8.89 \mathrm{E}-10$ & $8.35 \mathrm{E}-08$ & 3.20E-09 \\
\hline F25 & $1.21 \mathrm{E}-12$ & $1.21 \mathrm{E}-12$ & $1.21 \mathrm{E}-12$ & $1.21 \mathrm{E}-12$ & $1.21 \mathrm{E}-12$ & $1.21 \mathrm{E}-12$ & $1.21 \mathrm{E}-12$ & $1.21 \mathrm{E}-12$ & $1.21 \mathrm{E}-12$ & $1.21 \mathrm{E}-12$ & $1.21 \mathrm{E}-12$ \\
\hline F26 & $1.21 \mathrm{E}-12$ & $1.21 \mathrm{E}-12$ & $1.21 \mathrm{E}-12$ & $1.21 \mathrm{E}-12$ & $1.21 \mathrm{E}-12$ & $1.21 \mathrm{E}-12$ & $1.21 \mathrm{E}-12$ & $1.21 \mathrm{E}-12$ & $1.21 \mathrm{E}-12$ & $1.21 \mathrm{E}-12$ & $1.21 \mathrm{E}-12$ \\
\hline F27 & $1.21 \mathrm{E}-12$ & $1.21 \mathrm{E}-12$ & $1.21 \mathrm{E}-12$ & $1.21 \mathrm{E}-12$ & $1.21 \mathrm{E}$ & $1.21 \mathrm{E}-12$ & $1.21 \mathrm{~F}$ & -12 & $1.21 \mathrm{E}-12$ & 1.21 & $1.21 \mathrm{E}-12$ \\
\hline F28 & 0.012732 & $1.17 \mathrm{E}-09$ & $5.07 \mathrm{E}-10$ & 0.001114 & $1.01 \mathrm{E}-08$ & $3.02 \mathrm{E}-11$ & $2.37 \mathrm{E}-10$ & $2.02 \mathrm{E}$ & $8.35 \mathrm{E}-08$ & 0.446419 & $2.71 \mathrm{E}-11$ \\
\hline F29 & $1.85 \mathrm{E}-08$ & $6.52 \mathrm{E}-09$ & 3.02E-11 & $1.29 \mathrm{E}-06$ & $7.12 \mathrm{E}-09$ & $3.02 \mathrm{E}-11$ & $1.17 \mathrm{E}-09$ & $3.02 \mathrm{E}-11$ & $3.02 \mathrm{E}-11$ & $2.6 \mathrm{E}-08$ & $3.02 \mathrm{E}-11$ \\
\hline
\end{tabular}




\section{Acknowledgments}

This research is funded by Zhejiang Provincial Natural Science Fr indation of China (LY17F020012), Science and Technology Plan Project of Wenzhou of Chin॰ (Zu`n17019).

We also acknowledge the comments of anonymous reviewers.

\section{References}

[1] R. Abbassi, A. Abbassi, A. A. Heidari, S. Mirjalili, An efficient salp swarm-inspired ; gorit __ for parameters identification of photovoltaic cell models, Energy Conversion and Management 179 (2019) 362-372.

[2] H. Faris, A. M. Al-Zoubi, A. A. Heidari, I. Aljarah, M. Mafarja, M. A. Hassonah, H. I. ita, An intelligent system for spam detection and identification of the most relevant features based on evolutionary rar suin weigh networks, Information Fusion 48 (2019) $67-83$.

[3] J. Nocedal, S. J. Wright, Numerical optimization 2nd, 2006.

[4] G. Wu, Across neighborhood search for numerical optimization, Information Srience mo 2016) 597-618.

[5] G. Wu, W. Pedrycz, P. N. Suganthan, R. Mallipeddi, A variable reduction st ttegy $f \sim r$ evolutionary algorithms handling equality constraints, Applied Soft Computing 37 (2015) 774-786.

[6] J. Dréo, A. Pétrowski, P. Siarry, E. Taillard, Metaheuristics for hard optimizauun: mf nods and case studies, Springer Science \& Business Media, 2006.

[7] E.-G. Talbi, Metaheuristics: from design to implementation, volume 74, John w. 'ey \& Sons, 2009.

[8] S. Kirkpatrick, C. D. Gelatt, M. P. Vecchi, Optimization by simulatec' nnealing. science 220 (1983) 671-680.

[9] J. H. Holland, Genetic algorithms, Scientific american 267 (1992) $6 f-73$.

[10] J. Luo, H. Chen, Y. Xu, H. Huang, X. Zhao, et al., An improved grasshe ner optimization algorithm with application to financial stress prediction, Applied Mathematical Modelling 64 (2018) 654-668.

[11] M. Wang, H. Chen, B. Yang, X. Zhao, L. Hu, Z. Cai, H. Huang, Tong, Toward an optimal kernel extreme learning machine using a chaotic moth-flame optimization strategy with applications in - edical diagnoses, Neurocomputing 267 (2017) 69-84.

[12] L. Shen, H. Chen, Z. Yu, W. Kang, B. Zhang, H. Li, B. Yang, ' ' - F. Folving support vector machines using fruit fly optimization for medical data classification, Knowledge-Based Systems 96 (. $716^{`} 61-75$.

[13] Q. Zhang, H. Chen, J. Luo, Y. Xu, C. Wu, C. Li, Chaos enhance bacterial foraging optimization for global optimization, IEEE Access (2018).

[14] A. A. Heidari, R. A. Abbaspour, A. R. Jordehi, An efficient ch otı vater cycle algorithm for optimization tasks, Neural Computing and Applications 28 (2017) 57-85.

[15] M. Mafarja, I. Aljarah, A. A. Heidari, A. I. Hammourı, ' Haw A.-Z. AlaM, S. Mirjalili, Evolutionary population dynamics and grasshopper optimization approaches for feature selection pı hlems, Knowledge-Based Systems 145 (2018) $25-45$.

[16] M. Mafarja, I. Aljarah, A. A. Heidari, H. Faris, P. Fournier-Viger, X. Li, S. Mirjalili, Binary dragonfly optimization for feature selection using time-varying transfer functions, now. Ige-Based Systems 161 (2018) $185-204$.

[17] I. Aljarah, M. Mafarja, A. A. Heidari, H. Far s, Y. Zhc ig, S. Mirjalili, Asynchronous accelerating multi-leader salp chains for feature selection, Applied Soft Computing $71(<18) 96<-979$.

[18] S. Mirjalili, A. Lewis, The whale optimizat: nn algoı. '. Advances in Engineering Software 95 (2016) 51-67.

[19] H. Faris, M. M. Mafarja, A. A. Heidari, I Alja sh, A.-Z. AlaM, S. Mirjalili, H. Fujita, An efficient binary salp swarm algorithm with crossover scheme for feature selectic nr slem Knowledge-Based Systems 154 (2018) 43-67.

[20] J. R. Koza, Genetic Programming II, f utomı : $\Gamma$ scovery of Reusable Subprograms, MIT Press, Cambridge, MA, 1992.

[21] R. Storn, K. Price, Differential evolu ${ }^{+}$on-a simpı and efficient heuristic for global optimization over continuous spaces, Journal of global optimization 11 (1997) 341 35s

[22] D. Simon, Biogeography-based optimization, EEE transactions on evolutionary computation 12 (2008) 702-713.

[23] O. K. Erol, I. Eksin, A new optir .tZa on method: big bang-big crunch, Advances in Engineering Software 37 (2006) $106-111$.

[24] R. A. Formato, Central force or imiz tion, progress in Electromagnetic Research77 (2007) 425-491.

[25] E. Rashedi, H. Nezamabadi-Pou, ` jaryazdi, Gsa: a gravitational search algorithm, Information sciences 179 (2009) $2232-2248$.

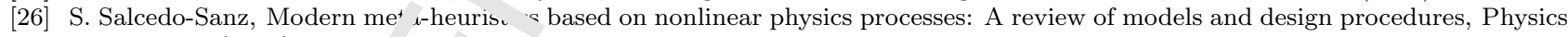
Reports 655 (2016) 1-70.

[27] F. Glover, Tabu searchpa i. r RSA journal on computing 1 (1989) 190-206.

[28] M. Kumar, A. J. Kulkarni, S. \ S tapathy, Socio evolution \& learning optimization algorithm: A socio-inspired optimization methodology, Future $C$-neration computer Systems 81 (2018) 252-272.

[29] R. V. Rao, V. J. Savs ni, D. V. 'haria, Teaching-learning-based optimization: an optimization method for continuous non-linear large scale problems, . 'formati a Sciences 183 (2012) 1-15.

[30] A. Baykasoğlu, F. R $\mathrm{O}_{4} \quad . . .1$, Evolutionary and population-based methods versus constructive search strategies in dynamic combinatorial of Imizati $n$, Information Sciences 420 (2017) 159-183.

[31] A. A. Heidari, I. Faris, Aljarah, S. Mirjalili, An efficient hybrid multilayer perceptron neural network with grasshopper optimization, So Compr ing (2018) 1-18.

[32] R. Eberhart. J. Kenıuy, A new optimizer using particle swarm theory, in: Micro Machine and Human Science, 1995. MHS'95., Proceedings $1 \mathrm{I}$ un $\cdots+\mathrm{h}$ International Symposium on, IEEE, pp. 39-43.

[33] M. Dorigo, V. 'I Aiezzo, A. Colorni, Ant system: optimization by a colony of cooperating agents, IEEE Transactions on Systems, Man, and Cyber otics, Part B (Cybernetics) 26 (1996) 29-41.

[34] A. H. Gandomi, ג.-S. Yang, A. H. Alavi, Cuckoo search algorithm: a metaheuristic approach to solve structural optimization problems, Engineering with computers 29 (2013) 17-35.

[35] X.-S. Yang, Review of meta-heuristics and generalised evolutionary walk algorithm, International Journal of Bio-Inspired Computation 3 (2011) 77-84. 
[36] D. H. Wolpert, W. G. Macready, No free lunch theorems for optimization, IEEE transactions on evolutionary computation 1 (1997) 67-82.

[37] J. C. Bednarz, Cooperative hunting in harris' hawks (parabuteo unicinctus), Science 239 (1988) 152 ?

[38] L. Lefebvre, P. Whittle, E. Lascaris, A. Finkelstein, Feeding innovations and forebrain size in birds _. imal Behaviour 53 (1997) $549-560$.

[39] D. Sol, R. P. Duncan, T. M. Blackburn, P. Cassey, L. Lefebvre, Big brains, enhanced cognitior ר response of birds to novel environments, Proceedings of the National Academy of Sciences of the United States of Amerir i 10\% (2005) 5460-5465.

[40] F. Dubois, L.-A. Giraldeau, I. M. Hamilton, J. W. Grant, L. Lefebvre, Distraction sneakers decr - se t’ ¿ expected level of aggression within groups: a game-theoretic model, The American Naturalist 164 (2004) E32-E45.

[41] EurekAlertAAAS, Bird iq test takes flight, 2005.

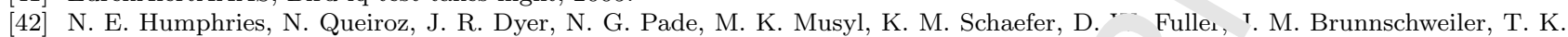
Doyle, J. D. Houghton, et al., Environmental context explains lévy and brownian moven nt p r rns of marine predators, Nature 465 (2010) 1066-1069.

[43] G. M. Viswanathan, V. Afanasyev, S. Buldyrev, E. Murphy, P. Prince, H. E. Stanley, Le $r$ flight search patterns of wandering albatrosses, Nature 381 (1996) 413.

[44] D. W. Sims, E. J. Southall, N. E. Humphries, G. C. Hays, C. J. Bradshaw, J. V. Pitch ord, A. James, M. Z. Ahmed, A. S. Brierley, M. A. Hindell, et al., Scaling laws of marine predator search behaviour, - ature 45 (2008) 1098-1102.

[45] A. O. Gautestad, I. Mysterud, Complex animal distribution and abundance from ment 3 (2006) 44-55.

[46] M. F. Shlesinger, Levy flights: Variations on a theme, Physica D: Nonlinear Phe' Jme a 38 (1989) $304-309$.

[47] G. Viswanathan, V. Afanasyev, S. V. Buldyrev, S. Havlin, M. Da Luz, E. Rapı v, H. F Stanley, Lévy flights in random searches, Physica A: Statistical Mechanics and its Applications 282 (2000) 1-12.

[48] X.-S. Yang, Nature-inspired metaheuristic algorithms, Luniver press, 2010.

[49] X. Yao, Y. Liu, G. Lin, Evolutionary programming made faster, IEEE ? -nsaction on Evolutionary computation 3 (1999) 82-102.

[50] J. G. Digalakis, K. G. Margaritis, On benchmarking functions for g netı ' 'oor' nms, International journal of computer mathematics 77 (2001) 481-506.

[51] S. García, D. Molina, M. Lozano, F. Herrera, A study on the use of non-nar r. etric tests for analyzing the evolutionary algorithms behaviour: a case study on the cec2005 special session on real pa. meter optimization, Journal of Heuristics 15 (2009) 617.

[52] X.-S. Yang, A. Hossein Gandomi, Bat algorithm: a novel approach for ¿'vbal engineering optimization, Engineering Computations 29 (2012) 464-483.

[53] X.-S. Yang, M. Karamanoglu, X. He, Flower pollination algoric m: nuvel approach for multiobjective optimization, Engineering Optimization 46 (2014) 1222-1237.

[54] A. H. Gandomi, X.-S. Yang, A. H. Alavi, Mixed variable s - ${ }^{+}$uraı ptimization using firefly algorithm, Computers \& Structures 89 (2011) 2325-2336.

[55] S. Mirjalili, S. M. Mirjalili, A. Lewis, Grey wolf optiminer, Aa ances in Engineering Software 69 (2014) 46-61.

[56] S. Mirjalili, Moth-flame optimization algorithm: A nove natuı nspired heuristic paradigm, Knowledge-Based Systems 89 (2015) $228-249$.

[57] J. Derrac, S. García, D. Molina, F. Herrera, A practical tutorial on the use of nonparametric statistical tests as a methodology

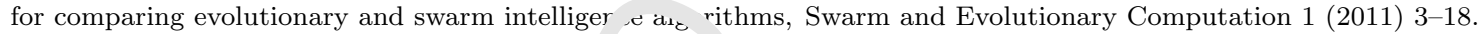

[58] X.-S. Yang, Firefly algorithm, stochastic test fu ctions ar. ! design optimisation, International Journal of Bio-Inspired Computation 2 (2010) 78-84.

[59] F. Van Den Bergh, A. P. Engelbrecht, A udy oı c cicle swarm optimization particle trajectories, Information sciences 176 (2006) 937-971.

[60] S. Mirjalili, A. H. Gandomi, S. Z. Mirja! : S. jarer ., H. Faris, S. M. Mirjalili, Salp swarm algorithm: A bio-inspired optimizer for engineering design problems, Advar es lı. Tng; eering Software (2017).

[61] H. Eskandar, A. Sadollah, A. Bahrei nejad, M. Aamdi, Water cycle algorithm-a novel metaheuristic optimization method for solving constrained engineering opti iiza ’ n problems, Computers \& Structures 110 (2012) 151-166.

[62] S. Saremi, S. Mirjalili, A. Lewis, Grasshoppt_ ptimisation algorithm: Theory and application, Advances in Engineering Software 105 (2017) 30-47.

[63] C. A. C. Coello, Use of a self-ad stiv penalty approach for engineering optimization problems, Computers in Industry 41 (2000) $113-127$.

[64] M. Zhang, W. Luo, X. Wang Differ +ial evolution with dynamic stochastic selection for constrained optimization, Information Sciences 178 (2008) 3043-3r 14.

[65] S. Mirjalili, S. M. Mirjali A. Hata ılou, Multi-verse optimizer: a nature-inspired algorithm for global optimization, Neural Computing and Applications - (20 6) 495-513.

[66] H. Liu, Z. Cai, Y. W . . Hybr...zing particle swarm optimization with differential evolution for constrained numerical and engineering optimizat on, App ad Soft Computing 10 (2010) 629-640.

[67] A. Sadollah, A. Bahr, ninejad, A. Eskandar, M. Hamdi, Mine blast algorithm: A new population based algorithm for solving constrained engineering _. +im Lation problems, Applied Soft Computing 13 (2013) 2592-2612.

[68] J.-F. Tsai, Glob + optimization of nonlinear fractional programming problems in engineering design, Engineering Optimization 37 (2005) 399-4 9.

[69] T. Ray, P. Sain, Engin sing design optimization using a swarm with an intelligent information sharing among individuals, Engineering Optimı _l 33 (2001) 735-748.

[70] A. Kaveh, L A novel meta-heuristic optimization algorithm: Thermal exchange optimization, Advances in Engineering Software $110,0^{\prime}$ ') $69-84$.

[71] H. Salimi, Stocı. stic fractal search: a powerful metaheuristic algorithm, Knowledge-Based Systems 75 (2015) 1-18.

[72] J. S. Arora, Intro uction to optimum design, 1989, McGraw-Mill Book Company (1967).

[73] K. Deb, Optimal design of a welded beam via genetic algorithms, AIAA journal 29 (1991) $2013-2015$.

[74] C. A. C. Coello, E. M. Montes, Constraint-handling in genetic algorithms through the use of dominance-based tournament selection, Advanced Engineering Informatics 16 (2002) 193-203. 
[75] A. D. Belegundu, J. S. Arora, A study of mathematical programming methods for structural optimization. part i: Theory, International Journal for Numerical Methods in Engineering 21 (1985) 1583-1599.

[76] Q. He, L. Wang, An effective co-evolutionary particle swarm optimization for constrained engineering d sign problems, Engineering Applications of Artificial Intelligence 20 (2007) 89-99.

[77] L. Wang, L.-p. Li, An effective differential evolution with level comparison for constrained engiı sering - sign, Structural and Multidisciplinary Optimization 41 (2010) 947-963.

[78] Y. Wang, Z. Cai, Y. Zhou, Z. Fan, Constrained optimization based on hybrid evolutionary a', orit $n$ and adaptive constrainthandling technique, Structural and Multidisciplinary Optimization 37 (2009) 395-413.

[79] A. Kaveh, T. Bakhshpoori, Water evaporation optimization: a novel physically inspired ortimiza ' ’n algorithm, Computers \& Structures 167 (2016) 69-85.

[80] A. H. Gandomi, X.-S. Yang, A. H. Alavi, S. Talatahari, Bat algorithm for constrained opt' ' 'ation 'sks, Neural Computing and Applications 22 (2013) 1239-1255.

[81] E. Mezura-Montes, C. A. C. Coello, A simple multimembered evolution strategy tc olve constrained optimization problems, IEEE Transactions on Evolutionary computation 9 (2005) 1-17.

[82] W. Gong, Z. Cai, D. Liang, Engineering optimization by means of an improved unstraineu differential evolution, Computer Methods in Applied Mechanics and Engineering 268 (2014) 884-904.

[83] Q. He, L. Wang, A hybrid particle swarm optimization with a feasibility-base rule for constrained optimization, Applied mathematics and computation 186 (2007) 1407-1422.

[84] L. dos Santos Coelho, Gaussian quantum-behaved particle swarm optimize ton annroaches for constrained engineering design problems, Expert Systems with Applications 37 (2010) 1676-1683.

[85] H. Rosenbrock, An automatic method for finding the greatest or least valu of a $f$ nction, The Computer Journal 3 (1960) $175-184$.

[86] A. Kaveh, S. Talatahari, A novel heuristic optimization method: charged systeı search, Acta Mechanica 213 (2010) $267-289$.

[87] M. Montemurro, A. Vincenti, P. Vannucci, The automatic dynamic enalisatis I method (adp) for handling constraints with genetic algorithms, Computer Methods in Applied Mechanics and Engine ' ‘n ? ,6 (2013) 70-87.

[88] E. Mezura-Montes, C. Coello Coello, J. Velázquez-Reyes, L. Muñoz-ı 'vila, Multiple trial vectors in differential evolution for engineering design, Engineering Optimization 39 (2007) 567-589.

[89] K. Ragsdell, D. Phillips, Optimal design of a class of welded struc res using geometric programming, Journal of Engineering for Industry 98 (1976) 1021-1025.

[90] K. S. Lee, Z. W. Geem, A new structural optimization methr ' ' cor on the harmony search algorithm, Computers \& structures $82(2004) 781-798$.

[91] F.-z. Huang, L. Wang, Q. He, An effective co-evolutionary differer ^el evolution for constrained optimization, Applied Mathematics and computation 186 (2007) 340-356.

[92] P. Savsani, V. Savsani, Passing vehicle search (pvs): A noveı ne 'euristic algorithm, Applied Mathematical Modelling 40 (2016) 3951-3978.

[93] R. V. Rao, V. J. Savsani, D. Vakharia, Teaching-learn. ${ }^{r}$-basun optimization: a novel method for constrained mechanical design optimization problems, Computer-Aided Design 43 (2011) - ’?-315.

[94] S. Gupta, R. Tiwari, S. B. Nair, Multi-objective design optimisation of rolling bearings using genetic algorithms, Mechanism and Machine Theory 42 (2007) 1418-1443. 


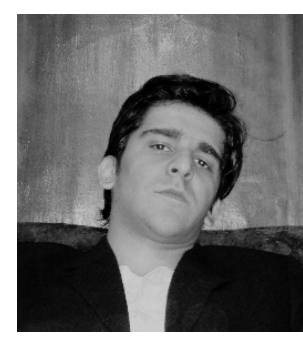

Systems.

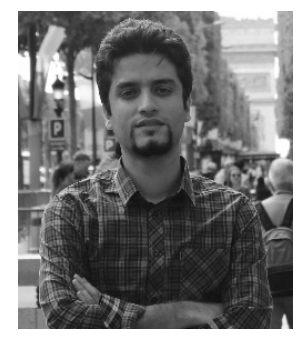

Seyedali Mirjalili is a lecturer at Griffith Ur ıverc, ${ }^{\circ}$, and internationally recognised for his advances in Swarm Intelligence (SI) and c stimisation, including the first set of SI techniques from a synthetic intelly nce standpoint - a radical departure from how natural systems are typicia." und rstood - and a systematic design framework to reliably benchmark, eva'rate, and propose computationally cheap robust optimisation algorithms. $\Gamma$........... has hablished over 80 journal articles, many in high-impact journals with ove 7000 citations in total with an H-index of 29 and G-index of 84. From Google Scholar mets -s, ic so globally the 3rd most cited researcher in Engineering Optimisation and Robust Optimis inn. ' 'e is serving an associate editor of Advances in Engineering Software and the journal of Algorith. 1 s.

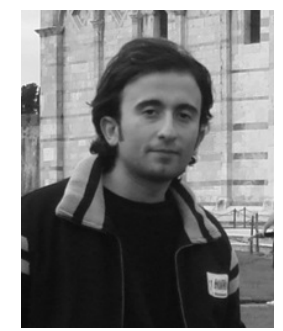

Hossam Faris is ar Assc iate professor at Business Information Technology department/King AL' 'ıllah II School for Information Technology/ The University of Jordan (Jorda 1). Jossam Faris received his BA, M.Sc. degrees (with excellent rates) in Compu'- Sc ence from Yarmouk University and Al-Balqa Applied University in '004 anu 2008 respectively in Jordan. Since then, he has been awarded a full-time com $_{\mathrm{r}}$ tition-based $\mathrm{PhD}$ scholarship from the Italian Ministry of Educatic 1 an $\mathrm{d}$ Research to peruse his $\mathrm{PhD}$ degrees in e-Business at University of Salento, Italy, where he o $n$ ' led his PhD degree in 2011. In 2016, he worked as a Postdoctoral researcher with $\mathrm{GeNev}$ a te` $\mathrm{m}$ aı the Information and Communication Technologies Research Center (CITIC), University e r ran da (Spain). His research interests include: Applied Computational Intelligence, Evolv wnary computation, Knowledge Systems, Data mining, Semantic Web and Ontologies. 


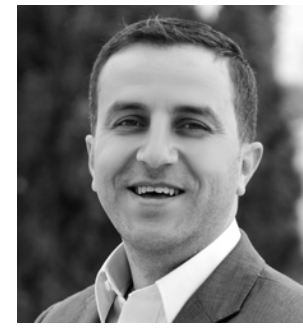

Ibrahim Aljarah is an assistant professor of BIG Data Mining a nd Computational Intelligence at the University of Jordan - Department of $\mathrm{B}_{\mu}$ iness Information Technology, Jordan. He obtained his bachelor degree in Computer Jcience from Yarmouk University - Jordan, 2003. Dr. Aljarah also ob dine ' his master degree in computer science and information systems from the , rrdan University of Science and Technology - Jordan in 2006. He also obtaineu 'is Ph.D. In computer Science from the North Dakota State University ( $N^{\top}$ JSI ', 'TSA, in May 2014. He organized and participated in many conferences in the field of data minı, machine learning, and Big data such as NTIT, CSIT, IEEE NABIC, CASON, and BIGD 1 A Congress. Furthermore, he contributed in many projects in USA such as Vehicle Class Detc tion $S$ stem (VCDS), Pavement Analysis Via Vehicle Electronic Telemetry (PAVVET), and $1 \mathrm{drm}$ uoud Storage System (CSS) projects. He has published more than 35 papers in refereed i te tati nal conferences and journals. His research focuses on data mining, Machine Learning, L. D...., MapReduce, Hadoop, Swarm intelligence, Evolutionary Computation, Social Network Analysis (SNA), and large scale distributed algorithms.

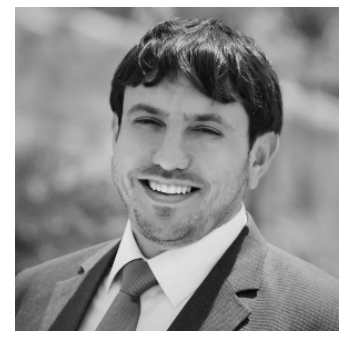

Majdi Mafarja received hl. $\iota S_{c}$ 1. Software Engineering and M.Sc in Computer Information Systems from I ' 'iladelphia University and The Arab Academy for Banking and Financial - -iences, Jordan in 2005 and 2007 respectively. Dr. Mafarja did his $\mathrm{PhN}$ in Computer Science at National University of Malaysia (UKM). He wa a men ber in Datamining and Optimization Research Group (DMO). Now he ${ }^{\prime} a^{+}$assistant professor at the Department of Computer Science at Sirz it Iniversity. His research interests include Evolutionary Computation, Meta-heuristics ar $\mathrm{d} L \mathrm{t}+\mathrm{r}$ ining.

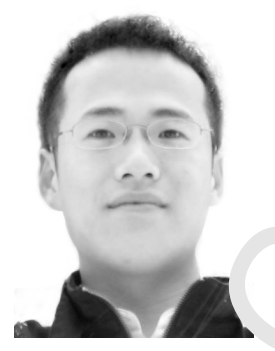

Huili g Chen is currently an associate professor in the department of computer scienc. it Venzhou University, China. He received his Ph.D. degree in the $\mathrm{d} r$ partmen of computer science and technology at Jilin University, China. His $\mathrm{p}$ esent $\mathrm{r}$ search interests center on evolutionary computation, machine learning, and Lata wuning, as well as their applications to medical diagnosis and bankruptcy pred stion. He has published more than 100 papers in international journals and $\cdots$. erence proceedings, including Pattern Recognition, Expert Systems with Application ? r w wledge-Based Systems, Soft Computing, Neurocomputing, Applied Mathematical Modeling, IE, E ACCESS, PAKDD, and among others. 
- A mathematical model is proposed to simulate the hunting behaviour of $\mathrm{H}_{\text {arris' }}$ Hawks

- An optimization algorithm is proposed using the mathematical model

- The proposed HHO algorithm is tested on several benchmarks

- The performance of HHO is also examined on several engineering c' ' '

- The results show the merits of the HHO algorithm as compared to ' $⿴$ e exı. ing algorithms 